\title{
De Novo Transcriptome Assembly of Isatis indigotica at Reproductive Stages and Identification of Candidate Genes Associated with Flowering Pathways
}

\author{
Yu Bai, Ying Zhou, Xiaoqing Tang ${ }^{1}$, and Yu Wang \\ College of Horticulture, Nanjing Agricultural University, Tongwei Road 6, Nanjing, 210095, People's \\ Republic of China

\begin{abstract}
Fangquan Wang and Jie Yang
Institute offood Crops, Jiangsu Academy Agricultural Sciences, Zhongling Street 48, Nanjing 210014, People's Republic of China
\end{abstract}

\begin{abstract}
Additional Index words. Illumina sequencing, functional annotation, tissue-specific transcriptome analysis, floweringassociated genes

Abstract. The appropriate timing of bolting and flowering is one of the keys to the reproductive success of Isatis indigotica. Several flowering regulatory pathways have been reported in plant species, but we know little about flowering regulatory in $I$. indigotica. In the present study, we performed RNA-seq and annotated I. indigotica transcriptome using RNA from five tissues (leaves, roots, flowers, fruit, and stems). Illumina sequencing generated $149,907,857$ high-quality clean reads and 124,508 unigenes were assembled from the sequenced reads. Of these unigenes, 88,064 were functionally annotated by BLAST searches against the public protein databases. Functional classification and annotation assigned 55,991 and 23,072 unigenes to 52 gene ontology (GO) terms and 25 clusters of orthologous group (COG) categories, respectively. A total of 19,927 unigenes were assigned to 124 Kyoto Encyclopedia of Genes and Genomes (KEGG) pathways, and 80 candidate genes related to plant circadian rhythm were identified. We also identified a number of differentially expressed genes (DEG) and 91 potential bolting and flowering-related genes from the RNA-seq data. This study is the first to identify bolting and flowering-related genes based on transcriptome sequencing and assembly in I. indigotica. The results provide foundations for the exploration of flowering pathways in I. indigotica and investigations of the molecular mechanisms of bolting and flowering in Brassicaceae plants.
\end{abstract}

Isatis indigotica, a biennial herbaceous plant belonging to the Brassicaceae family, is distributed widely across the Chinese mainland. The dried leaves and roots of the plant, which are also known as "Ban-Lan-Gen" and "Da-Qing-Ye" in China, have commonly been used as medicines for hundreds of years to treat mumps, febrile diseases, eruptive diseases, sore throat, and inflammatory diseases (National Pharmacopoeia Committee, 2015). Bolting and flowering are crucial developmental stages in the life cycle of $I$. indigotica. Premature bolting limits vegetative growth and reduces yield and quality of medicinal products of I. indigotica.

The formation of bolting and flowering is of great importance in plant life cycle and marks the transition from vegetative growth to reproductive development. A proper bolting and flowering time is crucial for reproductive success and high productivity (Amasino and Michaels, 2010; Srikanth and Schmid, 2011). The interplays of central flowering genes regulate the transition from vegetative growth to reproductive development (Moon et al., 2005; Parcy, 2005). The genes involved in flowering control have been characterized in Arabidopsis thaliana and have been integrated into multiple flowering pathways, including vernalization, aging, photoperiod,

Received for publication 21 Aug. 2017. Accepted for publication 4 Dec. 2017. This work was supported by the National Natural Science Foundation of China (Grant no. 31171486).

${ }^{1}$ Corresponding author. E-mail: xqtang@njau.edu.cn. the gibberellin (GA), and autonomous pathways (Amasino and Michaels, 2010; Srikanth and Schmid, 2011; Wang, 2014). In recent years, functional genes and regulatory pathways related to flowering time have also been investigated in many crops, including maize [Zea mays (Dong et al., 2012)], rice [Oryza sativa (Shrestha et al., 2014; Tsuji et al., 2011)], strawberry [Fragaria Xananassa (Sánchez-Sevilla et al., 2014)], and soybean [Glycine max (Jung et al., 2012)].

RNA-seq is an important tool for obtaining transcripts from certain plant tissues under specific physiological conditions or at specific developmental stages (Strickler et al., 2012; Wang et al., 2009). Illumina (San Diego, CA) sequencing techniques have increased gene discoveries in the life sciences in recent years (Zhang et al., 2013). RNA-seq technology has been extensively used in transcriptome analyses of model plant species, such as $A$. thaliana (Zhu et al., 2013), maize (Dukowic-Schulze et al., 2014; Thakare et al., 2014), soybean (Stamm et al., 2014), rice (Huang et al., 2014), and non-model plant species, such as wild strawberry [Fragaria vesca (Mouhu et al., 2009)] and radish [Raphanus sativus (Nie et al., 2016)]. Tang et al. (2014) conducted transcriptome sequencing of $I$. indigotica by using Illumina technology to determine the genes involved in the biosynthesis of the active ingredient and its derivatives at the vegetative growth stage. There is still a lack of studies of the molecular mechanisms of bolting and flowering regulation of I. indigotica, and further transcriptome sequencing studies of I. indigotica at reproductive stages are needed. 
In this study, Illumina technology was used to sequence mRNAs of I. indigotica from various tissues (flowers, leaves, stems, roots, and fruit) to investigate the molecular mechanisms of bolting and flowering regulation of I. indigotica. On the basis of unigene assembly and analysis of DEGs, several candidate genes related to the flowering pathway were analyzed by quantitative real time polymerase chain reaction (qRT-PCR). The results of this study will enhance our understanding of the bolting and flowering-time regulatory networks in I. indigotica and provide clues for further investigations on the molecular genetic mechanisms underlying bolting and flowering regulation in the Brassicaceae plants.

\section{Materials and Methods}

Plant materials AND RNA isolation. The experiment was carried out at the greenhouse of Nanjing Agricultural University (Nanjing, China). The seeds of I. indigotica cultivar Bozhou was sown in plastic pots (29.6-cm diameter, 19.7-cm height) containing a compost of humus and vermiculite at a ratio of $1: 1$ on 8 Sept. 2015. Plants were grown under a natural light condition at $25{ }^{\circ} \mathrm{C}$ maximum and $-2{ }^{\circ} \mathrm{C}$ minimum with $60 \%$ to $75 \%$ air humidity. The plants were irrigated with distilled water once in every $5 \mathrm{~d}$ until they were sampled. The plants were sampled when some fruit appeared and flowering was still lasting. A total of $500 \mathrm{mg}$ of different tissues of I. indigotica, including roots, stems, leaves, fruit, and flowers were collected on 7 Apr. 2016 and then stored at $-80{ }^{\circ} \mathrm{C}$ until analyzed. Three biological replicates from distinct plants were harvested for each tissue. Total RNA was extracted from each tissue using the Trizol plus kit (Biouniquer, Nanjing, China) and treated with DNase I to remove contaminated DNA. We used the 2100 Bioanalyzer to analyze the quality and integrity of the DNase I-treated RNA (Agilent Technologies, San Francisco, CA).

RNA-SEQ LIBRARY CONSTRUCTION AND SEQUENCING. mRNAS were purified from total RNA using the Oligotex mRNA Midi Kit (Qiagen, Dusseldorf, Germany) and quantified using a spectrophotometer (Nano-Drop 2000; Thermo Scientific, Waltham, MA) and used to generate the cDNA library according to the Illumina manufacturer's instructions. Briefly, fragmentation buffer was added to interrupt mRNA to short fragments. Random hexamer primers were added to these short fragments to synthesize the first-strand cDNA. The secondstrand cDNA was synthesized using the Super-Script doublestranded cDNA synthesis kit (Invitrogen, Carlsbad, CA) and purified with a QiaQuick PCR extraction kit (Qiagen).

DE nOvo ASSEMbly OF TRANSCRIPTOME. Transcriptome de novo assembly was conducted using the Trinity program based on the de Bruijn graph algorithm (Grabherr et al., 2011). Clean reads were first assembled to form longer fragments named contigs. Based on the paired-end reads, different contigs from the same transcript sequences and the distance among them were detected and calculated. These contigs were then further assembled by the Trinity to generate unigenes which have no extension on either end. To quantify gene expression abundance, fragments per kilobase per transcript per million mapped reads (FPKM) was used (Mortazavi et al., 2008). The expression level of each unigene was calculated with the formula: FPKM $=\left(10^{6} \times C \times 10^{3}\right) / N L$, where $C$ is the number of reads that uniquely aligned to a certain unigene, $N$ is the total number of reads that uniquely aligned to all unigenes, and $L$ is the number of bases on this unigene.
Functional annotation and Classification. Unigenes were annotated against the publicly available protein databases NCBI nonredundant protein [NR (Polashock et al., 2010)], Swiss-Prot (Sato et al., 2006), COG (Natale et al., 2000), GO (Boyle et al., 2004), protein family [Pfam (Bateman et al., 2002)], euKaryotic ortholog groups [KOG (Li et al., 2003)], and KEGG (Wixon and Kell, 2000) using BLASTx with an $E$-value cutoff of $1.0 \mathrm{E}-05$. Based on the BLAST results, the coding sequence of unigenes were determined based on their orthologous proteins. If a unigene did not have a hit in any database, ESTScan software was used to find potential coding regions (Iseli et al., 1999), including the nucleotide $\left(5^{\prime}-3^{\prime}\right)$ and amino acid sequences of the coding regions. Based on the NR annotations, the Blast2GO program was used to gain GO annotations for the unigenes using a cutoff value of $1.0 \mathrm{E}-05$ at the second level according to molecular functions, cellular components, and biological processes (Conesa et al., 2005). GO functional classification and distribution of gene functions of each assembled unigene were performed using WEGO software at the macro level (Ye et al., 2006).

DifFERENTIAL EXPRESSION ANALYSIS. RNA-seq by expectation maximization, which allows for the assessment of transcript abundances based on the mapping of RNA-seq reads to the assembled transcriptome, was used for transcript abundance estimation of the de novo-assembled transcripts ( $\mathrm{Li}$ and Dewey, 2011). Differential expression analysis of two groups was performed using the DEGseq R package (1.10.1) (Wang et al., 2010). The false discovery rate (FDR) method was used to determine the threshold probability value in multiple tests. The probability values were adjusted using the BenjaminiHochberg procedure (Benjamini and Hochberg, 1995). If the FDR was smaller than $0.05($ FDR $\leq 0.01)$ and the absolute value of the $\log 2$ fold change was $\geq 2$, the unigene was considered to be a significant DEG. The Pearson correlation coefficient was calculated among the five samples according to the gene expression profiles.

GO enrichment analysis of the DEGs was implemented by the GOseq $\mathrm{R}$ packages based on the Wallenius noncentral

Table 1. Summary of the sequencing data from the five tissues of Isatis indigotica.

\begin{tabular}{lccc}
\hline Samples & Clean reads (no.) $)^{\mathrm{z}}$ & Clean data (no.) $^{\mathrm{y}}$ & GC content $(\%)^{\mathrm{x}}$ \\
\hline Flower & $33,718,037$ & $10,115,411,110$ & 47.37 \\
Fruit & $31,951,066$ & $9,585,319,800$ & 47.51 \\
Leaf & $28,623,805$ & $8,587,141,500$ & 47.57 \\
Root & $26,789,893$ & $8,036,967,900$ & 47.05 \\
Stem & $28,825,056$ & $8,647,516,800$ & 46.91 \\
\hline
\end{tabular}

${ }^{\mathrm{z}}$ Number of pair-end reads in clean data.

${ }^{\mathrm{y}}$ Number of nucleotides in clean data.

${ }^{\mathrm{x}}$ Ratio of guanine $(\mathrm{G})$ and cytimidine $(\mathrm{C})$ to total nucleotides.

Table 2. Summary of the transcriptome assembly of Isatis indigotica.

\begin{tabular}{lcc}
\hline & Transcript & Unigene \\
\hline Total no. & 176,074 & 124,508 \\
Total length (nt) ${ }^{\mathrm{z}}$ & $165,549,067$ & $99,942,890$ \\
N50 (nt) ${ }^{\mathrm{z}}$ & 1,338 & 1,027 \\
Mean length (nt) & 940.22 & 802.70 \\
Number of length $\geq 500 \mathrm{nt}$ & 105,783 & 64,954 \\
Number of length $\geq 2,000 \mathrm{nt}$ & 17,740 & 8,644 \\
\hline${ }^{\mathrm{z}} \mathrm{nt}=$ nucleotide; $\mathrm{N} 50=$ the median size of transcript/unigene.
\end{tabular}

$\mathrm{z}_{\mathrm{nt}}=$ nucleotide; N50 $=$ the median size of transcript/unigene. 


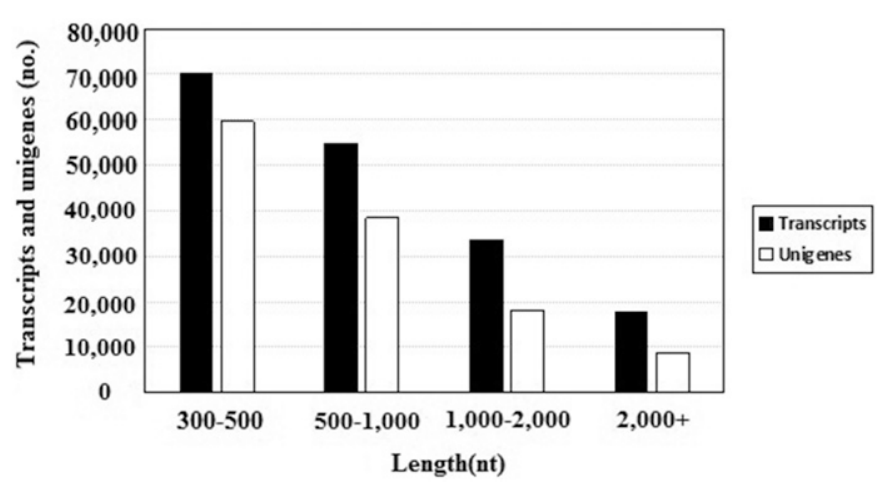

Fig. 1. The length distribution of assembled transcripts and unigenes in Isatis indigotica.

Table 3. Summary of functional annotation for Isatis indigotica unigenes.

\begin{tabular}{lcc}
\hline Database $^{z}$ & Unigenes (no.) & Percentage (\%) \\
\hline COG & 23,072 & 18.53 \\
GO & 55,991 & 44.97 \\
KEGG & 19,927 & 16.00 \\
KOG & 55,409 & 44.50 \\
Pfam & 50,154 & 40.28 \\
Swissprot & 55,189 & 44.33 \\
NR & 80,287 & 64.48 \\
Total & 88,064 & 70.73 \\
\hline
\end{tabular}

${ }^{\bar{z}} \mathrm{COG}=$ clusters of orthologous groups (Natale et al., 2000); $\mathrm{GO}=$ gene ontology (Boyle et al., 2004); KEGG = Kyoto Encyclopedia of Genes and Genomes (Wixon and Kell, 2000); KOG = euKaryotic ortholog groups (Li et al., 2003); Pfam = protein family (Bateman et al., 2002); $\mathrm{NR}=$ NCBI nonredundant protein (Polashock et al., 2010).

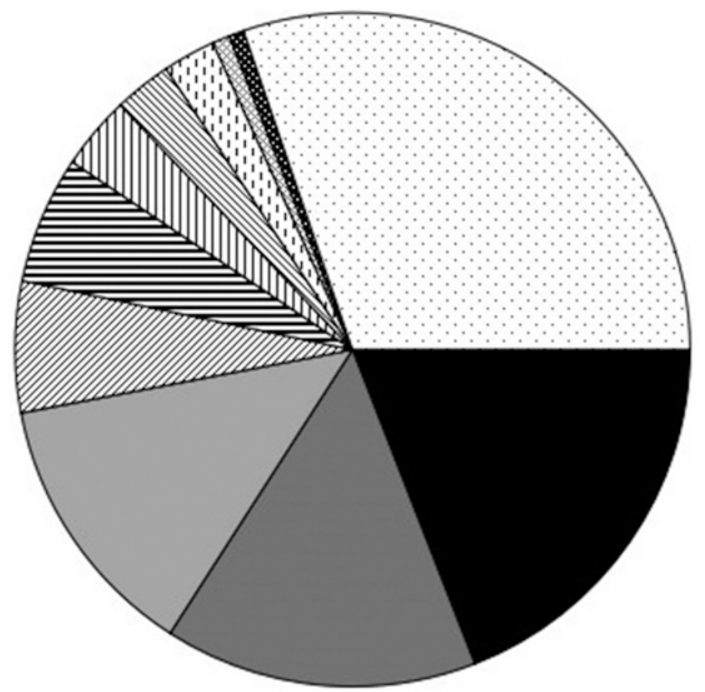

Eutrema salsugineum [15303]

口 Brassica napus [12016]

口Brassic rapa [10347]

Q Camelina sativa [5039]

E Arabidopsis thaliana [4906]

(10) Arabidopsis lyrata [2828]

A Arabis alpina [2208]

(1) Capsella rubella [2028]

Q Rhizophagus irregularis [664]

Q Mortierella verticillata [577]

Other [24233]

Fig. 2. Species distribution of the top BLAST hits against the NCBI nonredundant protein (Polashock et al., 2010) database.

hypergeometric distribution (Young et al., 2010). The numbers of all DEGs (upregulated and downregulated) were calculated for each GO term. In addition, KOBAS software was used to test the statistical enrichment of DEGs in KEGG pathways. The enrichment factor, which means the ratio of the DEGs number and the number of genes that have been annotated in this sed to represent enrichment intensiveness (Mao

RT-PCR ANALYSIS. qRT-PCR was performed to validate the relative expression levels of several flowering genes in different indigotica tissues. Total RNA from various tissue samples (PREMIER Biosoft Intl., Palo Alto, CA). Three ssion changes were calculated using the $2^{-\Delta \Delta C T}$ TransCrIPTOME SEQUENCING AND DE NOVO TRANSCRIPTOME -seq was performed using mRNA isolated from , indigotica in the transcripts. A total of $149,907,857$ high-quality clean reads of $44.97 \mathrm{Gbp}$ of sequences were obtained after ( the fewest $(26,789,893)$ number of clean reads, respectively (Table 1). The numbers of the reads for leaf and stem were $28,623,805$ and 28,825,056, respectively. There were 31,951,066 reads for fruit. These reads were used y using Trinity (Platel and Jain, 2012). A total of 176,074 transcripts with a median size (N50) of $1338 \mathrm{bp}$ and an average length of 940.22 bp were generated (Table 2); 124,508 unigenes were obtained with an N50 of $1027 \mathrm{bp}$ and an average length of $802.70 \mathrm{bp}$. Length distributions of assembled transcripts and unigenes were presented in Fig. 1. About $52 \%$ of the 124,508 unigenes were longer than $500 \mathrm{bp}$, and 8644 unigenes (6.94\%) were longer than $2 \mathrm{~kb}$ (Table 2).

FUNCTIONAL ANNOTATION OFI. INDIGOTICA TRANSCRIPTOME. To assign functional annotations of all the assembled unigenes, the sequence similarity search was performed against the NR, Swiss-Prot, COG, GO, Pfam, KOG, and KEGG public protein databases using a cutoff $E$-value of $1.0 \mathrm{E}-5$. Consequently, a total of 88,064 unigenes $(70.73 \%$ of all unigenes) found matches in at least one of the public databases (Table 3; Supplemental Table 1). Further blast searches showed that 23,072, 55,991, 50,154, $55,409,19,927,55,189$, and 80,287 unigenes had match in the COG, GO, Pfam, KOG, KEGG, Swissprot, and NR databases, respectively. 


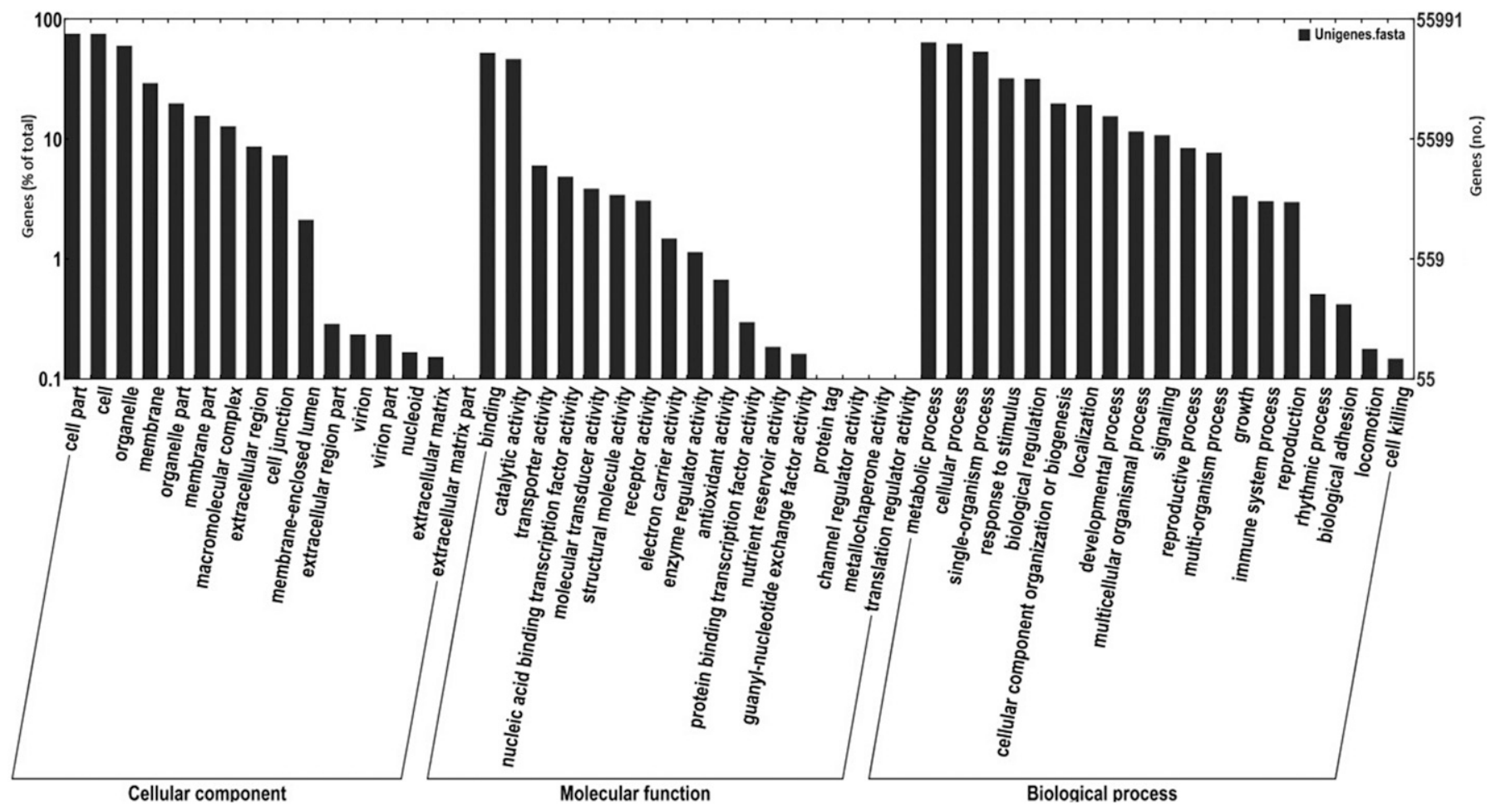

Fig. 3. Gene ontology (Boyle et al., 2004) classification of the Isatis indigotica unigenes.

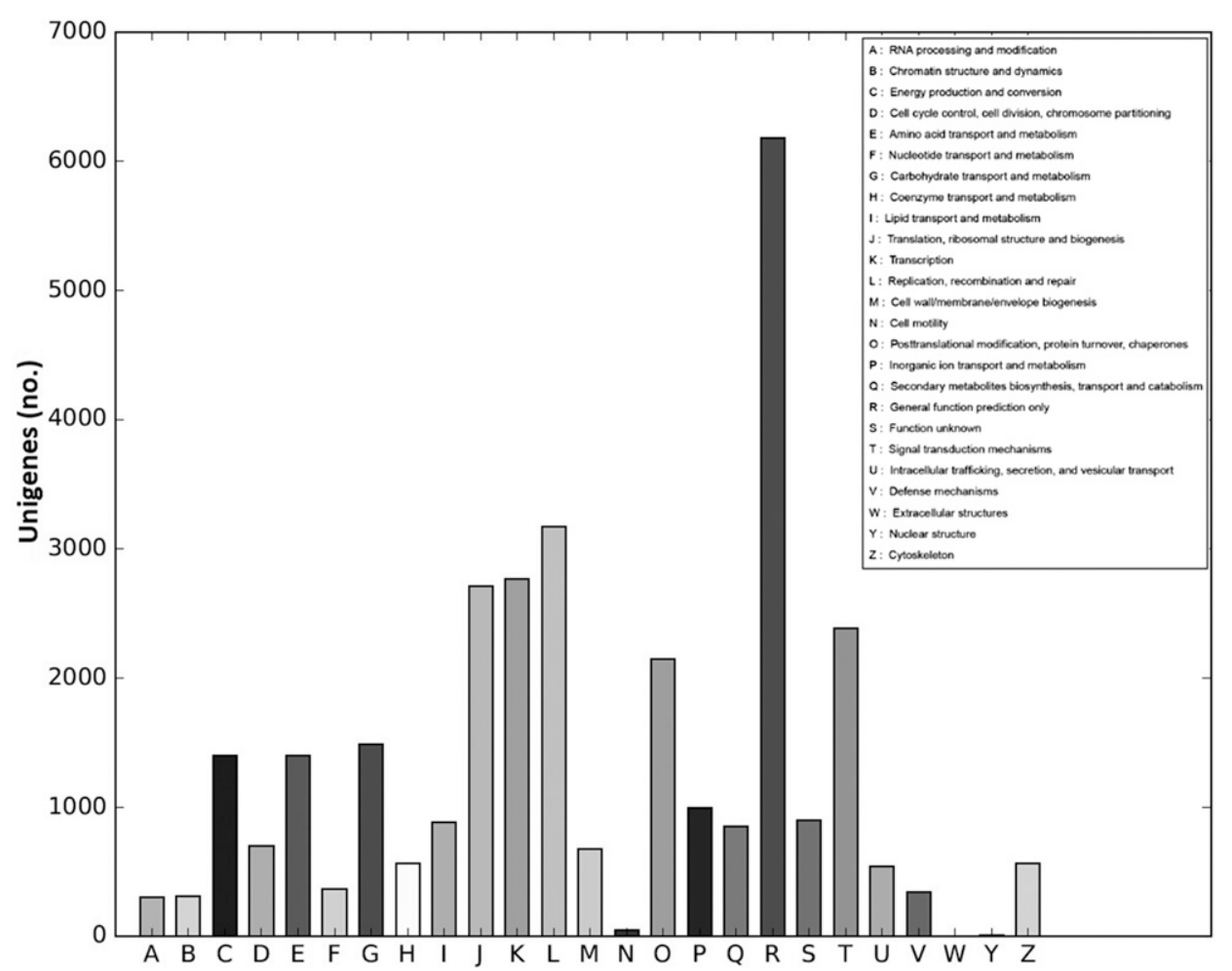

Fig. 4. Clusters of orthologous groups (Natale et al., 2000) functional classification of the Isatis indigotica unigenes.

Sequence similarity search against the NCBI NR database detected 80,287 annotated sequences ( $91.17 \%$ of all unigenes) that shared significant identity with known proteins (Table 3 ). The homologous species distribution is shown in Fig. 2. A total of $19.06 \%$ of the annotated sequences had matches to genes from Eutrema salsugineum, followed by Brassica napus (14.97\%), Brassica rapa $(12.89 \%)$, and Camelina sativa (6.28\%) (Fig. 2). The tophits were mostly Brassicaceae species, which indicated that the assembly and annotation of I. indigotica transcriptome are reliable.

GO AND COG Classification. To further explore the functions of the I. indigotica unigenes, GO analyses were performed and 55,991 unigenes were categorized into 52 GO classes including 16 cellular components, 17 molecular functions, and 19 biological processes (Fig. 3). Under the cellular components, the three largest percentages of genes were "cell part" $(42,536)$, "cell" $(42,415)$, and "organelle" $(33,702)$. Within the molecular function category, "binding" $(29,463)$, "catalytic activity", $(26,105)$, and "transporter activity" (3377) were the most highly represented categories. For the biological process category, the largest proportions of genes were clustered into "metabolic process" $(35,975)$, "cellular process" $(35,105$ sequences $)$, and "singleorganism process" $(30,137)$. However, only a few sequences came from the terms of "extracellular matrix part," 


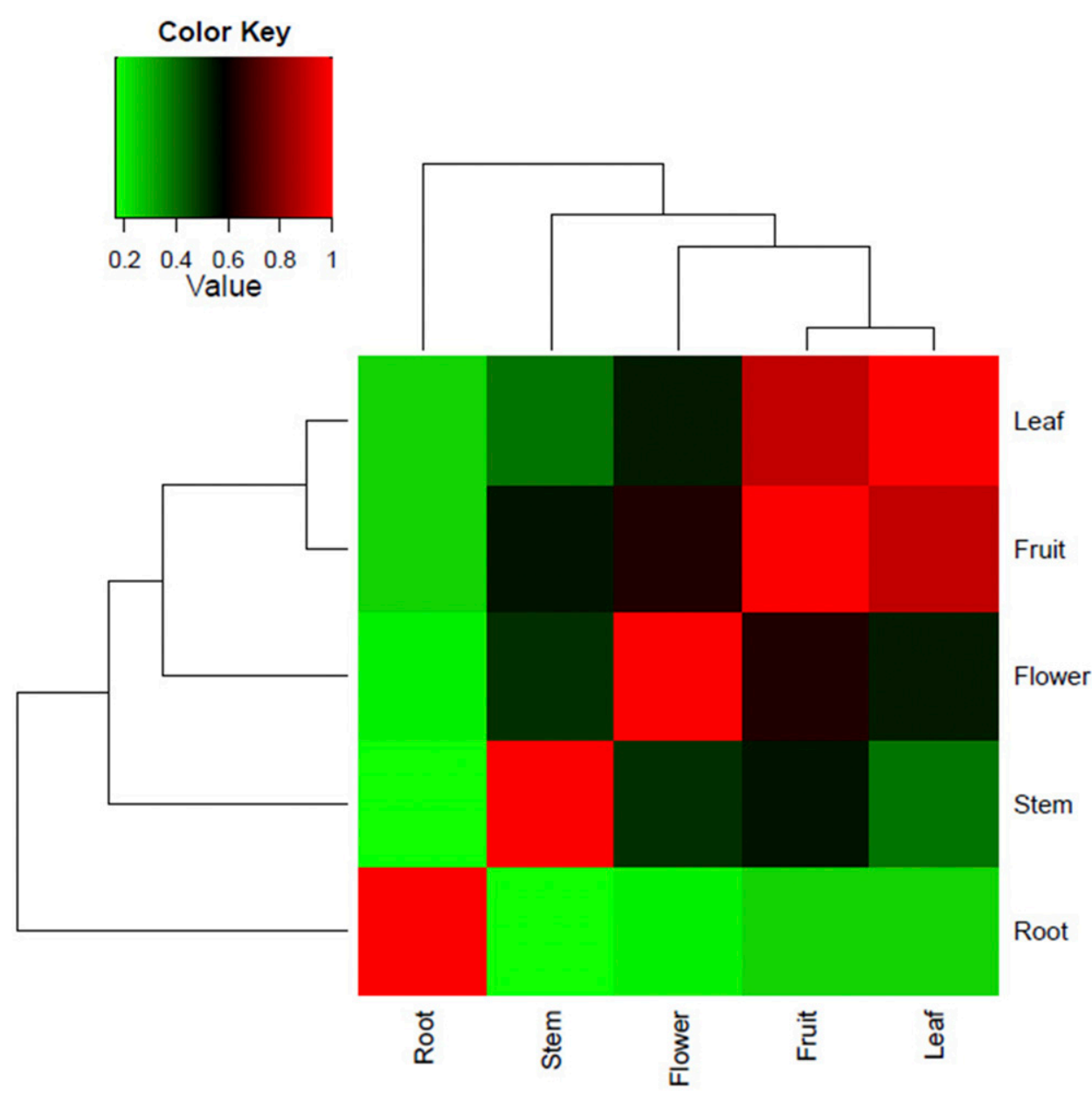

Fig. 5. The correlation dendrogram of expression patterns among the leaves, flowers, fruit, stems, and roots of Isatis indigotica.

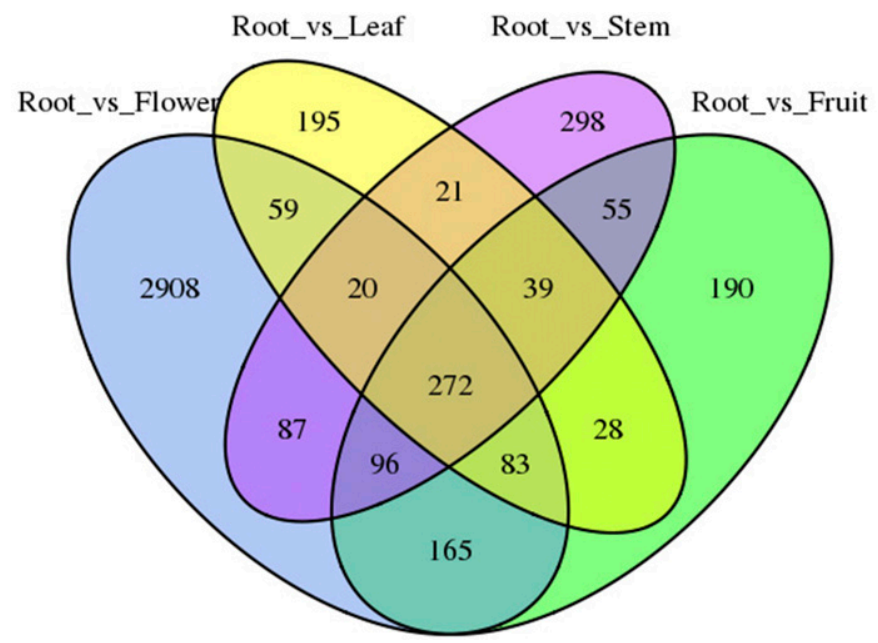

Fig. 6. Venn diagram of differentially expressed unigenes numbers among pairwise comparisons of flower, fruit, leaf, and stem with root.

"metallochaperone activity," and "translation regulator activity.",

In the COG classification, 23,072 unigenes were assigned to 25 COG categories (Table 3; Fig. 4). Of these, "general function prediction only" was dominant (6178 sequences), followed by "replication, recombination, and repair" (3175), "transcription" (2768), "translation, ribosomal structure and biogenesis" (2715), and "signal transduction mechanisms" (2390). Only a few genes matched the terms "extracellular structures" (1) and "nuclear structure" (9).

TISSUE-SPECIFIC TRANSCRIPTOME ANALYSIS AND IDENTIFICATION OF DEG IN I. INDigotica. The Pearson's distance correlation matrix, statistics of DEGs numbers, GO classification, and enriched $\mathrm{KEGG}$ pathways were used for comparative analysis. The RNA-Seq data were used to assess differences in the expression of genes in different tissues of I. indigotica, including leaves, flowers, fruit, stems, and roots. The FPKM values representing the expression levels of unigenes were calculated and compared among leaves, flowers, fruit, stems, and roots. The Pearson's distance correlation matrix was generated to compare the transcriptomes from each sample. The correlation dendrogram showed the relative relationships among the five tissues visually (Fig. 5). The results suggest that leaves were most similar to fruit, and roots were significantly different from other tissues (Fig. 5).

We compared the expression levels of unigenes from different tissues of I. indigotica (Fig. 6). A unigene was regarded as a DEG when the fold change was $\geq 2$ and the FDR was $<0.01$. There were 3690 DEGs between the flower and root, of which 3282 were downregulated, and 408 were upregulated (Table 4). In addition, there were 928 DEGs between the fruit and root, of which 784 were downregulated and 144 were upregulated. Moreover, we identified 717 DEGs between the leaf and root, 550 of which were downregulated and 167 of which were upregulated. Between the root and stem, 318 DEGs were downregulated whereas 570 DEGs were upregulated. Overall, we identified 272 common DEGs from the four comparison groups.

GO analysis of the DEGs in Flower_vs._Root showed that they were enriched in 52 categories, with cell part (1792 unigenes) representing the most abundant category, followed by cell (1783 unigenes), metabolic process (1607 unigenes), and cellular process (1579 unigenes) (Fig. 7). In Fruit_vs._Root, cell (640 unigenes) and cell part (639 unigenes) were the most abundant categories, followed by metabolic process (576 unigenes) and single-organism process (550 unigenes) (Supplemental Fig. 1). The most abundant categories in Leaf_vs._Root were similar to the categories in Fruit_vs._Root (Supplemental Fig. 2). The unigenes involved in cell (626 unigenes), cell part (626 unigenes), metabolic process (542 unigenes), cellular process (527 unigenes), organelle (519 unigenes), and singleorganism process (498 unigenes) were enriched between root and stem (Supplemental Fig. 3). 
Table 4. Summary of the differentially expressed genes (DEGs) numbers among pairwise comparisons of flower, fruit, leaf, and stem with root.

\begin{tabular}{lrcc}
\hline Comparison & DEG (no.) & Upregulated (no.) & Downregulated (no.) \\
\hline Flower_vs._Root & 3,690 & 408 & 3,282 \\
Fruit_vs._Root & 928 & 144 & 784 \\
Leaf_vs._Root & 717 & 167 & 550 \\
Root_vs._Stem & 888 & 570 & 318 \\
\hline
\end{tabular}

KEGG pathway analysis showed that the DEGs in fruit, flowers, leaves, and stems were significantly enriched when compared with roots in several pathways, including photosynthesis, stilbenoid, diarylheptanoid, and gingerol biosynthesis, photosynthesis-antenna proteins, carbon fixation in photosynthetic organisms, pentose and glucuronate interconversions, and glucosinolate biosynthesis (Table 5). Some pathways, particularly the pathways of energy metabolism and biosynthesis of other secondary metabolites, were highly overlapping

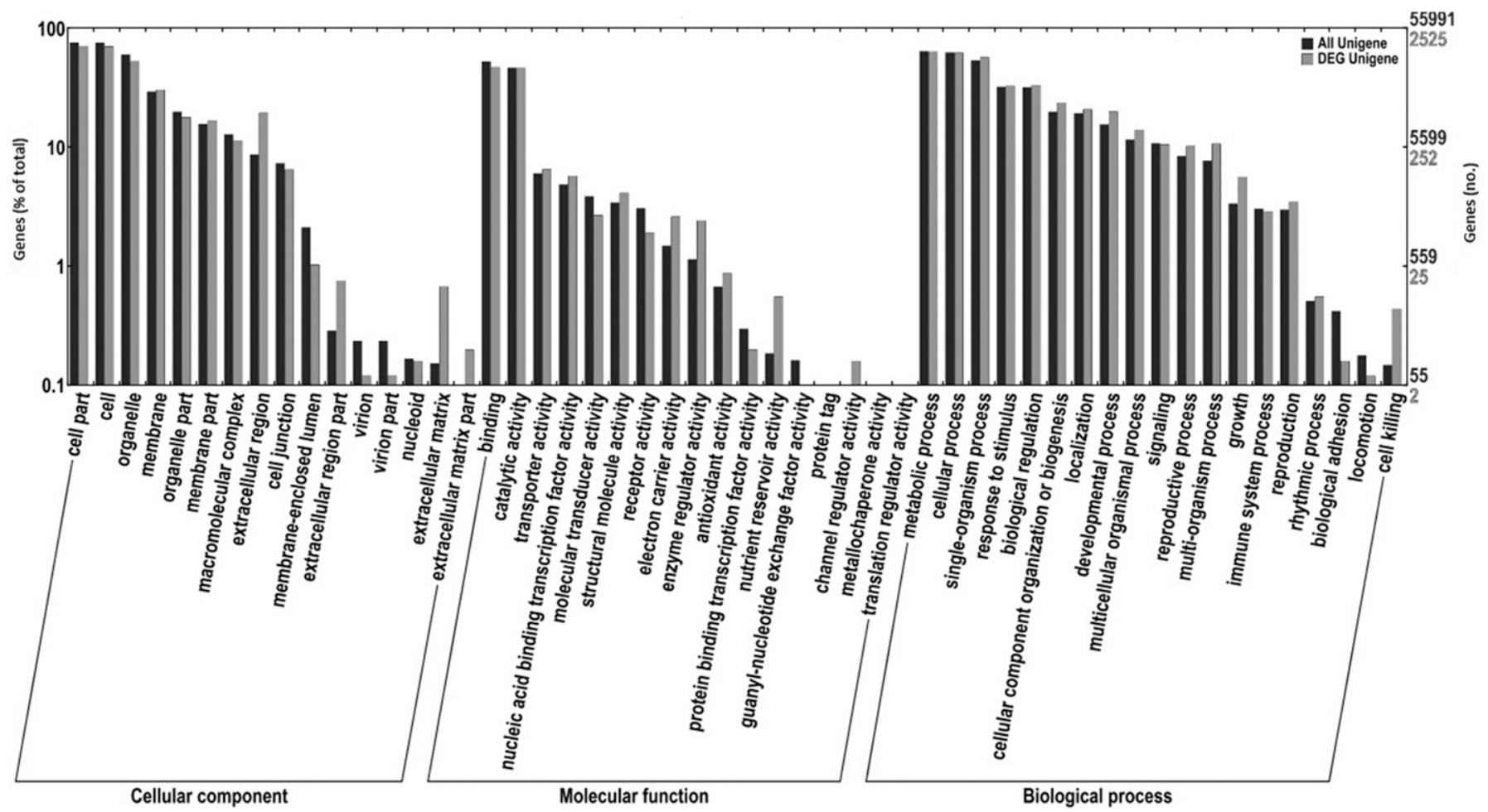

Fig. 7. Gene ontology (Boyle et al., 2004) classification of differentially expressed unigenes in Flower_vs._Root.

Table 5. Top four enriched Kyoto Encyclopedia of Genes and Genomes (Wixon and Kell, 2000) pathways among pairwise comparisons of flower, fruit, leaf, and stem with root.

\begin{tabular}{|c|c|c|c|c|}
\hline Comparison & Pathway terms & Enrichment factor $^{z}$ & $q$-value ${ }^{\mathrm{y}}$ & Unigenes (no.) $^{x}$ \\
\hline Flower_vs._Root & Pentose and glucuronate interconversions & 0.43 & 0.0098 & 24 \\
\hline \multirow[t]{3}{*}{ Fruit_vs._Root } & Photosynthesis & 0.072 & 0 & 27 \\
\hline & Photosynthesis-antenna proteins & 0.081 & $8.47 \mathrm{E}-8$ & 11 \\
\hline & Carbon fixation in photosynthetic organisms & 0.22 & $2.26 \mathrm{E}-6$ & 19 \\
\hline & Carbon fixation in photosynthetic organisms & 0.23 & $9.52 \mathrm{E}-6$ & 18 \\
\hline & Photosynthesis-antenna proteins & 0.096 & $1.52 \mathrm{E}-5$ & 9 \\
\hline & Monoterpenoid biosynthesis & 0.058 & 0.0051 & 4 \\
\hline \multirow[t]{2}{*}{ Root_vs._Stem } & Photosynthesis & 0.075 & 0 & 27 \\
\hline & Photosynthesis-antenna proteins & 0.12 & 0.0003 & 8 \\
\hline
\end{tabular}

${ }^{\mathrm{z}}$ Enrichment factor $=$ ratio of differentially expressed genes number and the number of genes have been annotated in this pathway.

${ }^{\mathrm{y}}$ Corrected probability value. A lower $q$-value means greater intensiveness.

${ }^{\mathrm{x}}$ Number of unigenes in each pathway. 


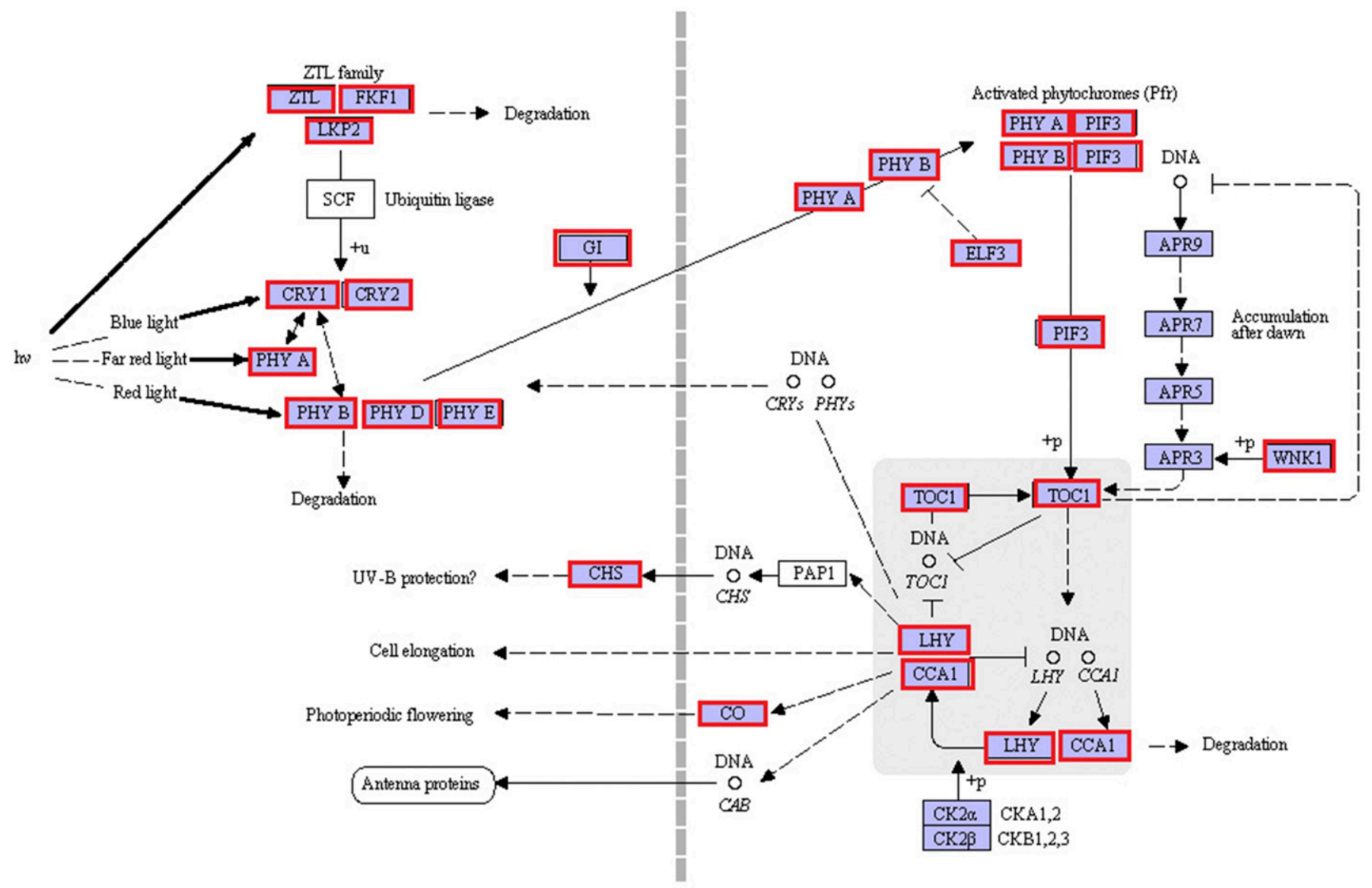

Fig. 8 . The pathway of circadian rhythm in Isatis indigotica . CCA1 $=$ circadian clock associated $1 ; \mathrm{CHS}=$ chalcone synthase; $\mathrm{CO}=$ constant; $\mathrm{CRY} 1=$ cryptochrome 1; CRY2 = cryptochrome 2; ELF3 = early flowering 3; FKF1 = flavin-binding kelch domain F box 1; GI = gigantea; LHY = late elongated hypocotyl; LKP2 = lov kelch protein 2; PHY A = phytochrome A; PHY B = phytochrome B; PHY D = phytochrome D; PHY E = phytochrome E; PIF3 = phytochrome interacting factor

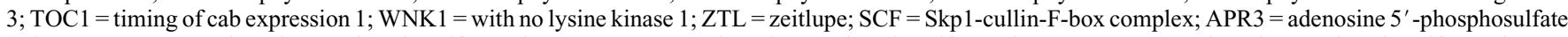
reductase 3; APR $5=$ adenosine 5 ' -phosphosulfate reductase 5; APR7 = adenosine 5 ' -phosphosulfate reductase 7; APR9= adenosine 5' -phosphosulfate reductase 9; CK2 $\alpha=$ Casein Kinase2 a-subunit; CK2 $\beta=$ Casein Kinase $2 \beta$-subunit; PAP1 = production of anthocyanin pigment 1; LKP2 = Lov Kelch Protein2; UV-B = ultraviolet-B; Pfr = far-red light absorption Phytochrome.

in all comparisons. For example, $\approx 30$ unigenes associated with photosynthesis were significantly enriched in flowers (Table 5). These results suggested that these genes were of great importance in the growth and development of I. indigotica.

KEGG PATHWAY ANALYSIS AND FUNCTIONAL GENES INVOLVED IN CIRCADIAN RHYTHM. To further analyze the transcriptome of I. indigotica, all unigenes were compared in the KEGG pathway database. In total, 19,927 unigenes were matched to the database and assigned to 124 KEGG pathways (Table 3; Supplemental Table 1). "Metabolic pathways" (ko01100: 5551 unigenes) had the largest number of unigenes, followed by "biosynthesis of secondary metabolites" (ko01110: 2552 unigenes), "ribosome" (ko03010: 1333 unigenes), "oxidative phosphorylation" (ko00190: 713 unigenes), and "spliceosome" (ko03040: 709 unigenes).

According to the pathway-based analysis, a total of 80 candidate genes were identified for the "circadian rhythm-plant" pathway (ko04712) (Fig. 8; Supplemental Tables 1 and 2). These rhythmrelated genes, including circadian clock associated 1 (CCA1, K12134), gigantea (GI, K12124), late elongated hypocotyl (LHY, K12133), flavin-binding kelch domain F box 1 (FKF1, K12116), phytochrome D (PHYD, K12122), cryptochrome 1 (CRY1, K12118), phytochrome A (PHYA, K12120), phytochrome B (PHYB, K12121), phytochrome E (PHYE, K12123), with no lysine kinase 1 (WNK1, K12132), phytochrome interacting factor 3 (PIF3, K12126), early flowering 3 (ELF3, K12125), lov kelch protein 2 (LKP2, K12117), and cryptochrome 2 (CRY2, K12119), were involved in many rhythmic processes such as photoperiodic flowering, cell elongation, and ultraviolet-B protection (Fig. 8). Besides, several genes, including constant (CO, K12135), zeitlupe (ZTL, K12115), timing of cab expression 1 (TOC1, K12127), and chalcone synthase (CHS, K00660), were implicated in plant light signaling pathways. Studying the roles of circadian-regulated genes responsible for controlling flowering could facilitate elucidation of interplays between photoperiod and the circadian clock.

IDENTIFICATION OF FLOWERING-ASSOCIATED GENES IN $\boldsymbol{I}$. INDIGOTICA. The complex genetic network which controls the development transition of flowering comprised several coordinate flowering pathways (Srikanth and Schmid, 2011). More than 200 flowering-related genes have been identified and characterized in the model plant $A$. thaliana (Amasino and Michaels, 2010; Srikanth and Schmid, 2011). To identify the genes responsible for bolting and flowering regulation in $I$. indigotica, a local BLASTx similarity search against $A$. thaliana flowering genes was performed. A total of 91 unigenes that showed high homology to the known bolting and flowering-related genes in A. thaliana were identified, and these 
Table 6. The identified candidate genes associated with flowering pathways of Isatis indigotica.

\begin{tabular}{|c|c|c|}
\hline Gene name ${ }^{z}$ & Unigenes (no.) & Flowering pathway \\
\hline$\overline{\mathrm{AP} 1}$ & 3 & Photoperiod \\
\hline AP2 & 1 & Photoperiod \\
\hline AGL6 & 1 & Photoperiod \\
\hline AGL8 & 1 & Photoperiod \\
\hline AGL14 & 1 & Others \\
\hline AGL15 & 1 & Photoperiod \\
\hline AGL16 & 1 & Others \\
\hline AGL17 & 1 & Others \\
\hline AGL18 & 1 & Photoperiod \\
\hline AGL19 & 1 & Vernalization \\
\hline CAL & 2 & Others \\
\hline CCA1 & 1 & Photoperiod/circadian clock \\
\hline $\mathrm{CO}$ & 2 & Photoperiod/circadian clock \\
\hline COL9 & 1 & Photoperiod/circadian clock \\
\hline CHS & 2 & Photoperiod \\
\hline CRY1 & 3 & Photoperiod/circadian clock \\
\hline CRY2 & 1 & Photoperiod/circadian clock \\
\hline EBS & 3 & Photoperiod \\
\hline ELF3 & 1 & Photoperiod/circadian clock \\
\hline ELF6 & 4 & Photoperiod \\
\hline ELF8 & 1 & Photoperiod \\
\hline ELF9 & 2 & Photoperiod \\
\hline EMF1 & 1 & Vernalization \\
\hline EMF2 & 1 & Vernalization \\
\hline ESD4 & 1 & Vernalization \\
\hline FD & 1 & Photoperiod \\
\hline FKF1 & 1 & Photoperiod \\
\hline FLC & 1 & integrator \\
\hline FLD & 1 & Autonomous \\
\hline FLK & 3 & Autonomous \\
\hline FPA & 1 & Autonomous \\
\hline FPF & 2 & GA \\
\hline FT & 1 & Integrator \\
\hline GI & 2 & Photoperiod/circadian clock \\
\hline HUA2 & 1 & Vernalization \\
\hline LD & 3 & Autonomous \\
\hline LHY & 1 & Photoperiod/circadian clock \\
\hline LKP2 & 1 & Photoperiod/circadian clock \\
\hline PHYA & 3 & Photoperiod/circadian clock \\
\hline PHYB & 1 & Photoperiod/circadian clock \\
\hline PHYD & 7 & Photoperiod/circadian clock \\
\hline PHYE & 1 & Photoperiod/circadian clock \\
\hline PIE1 & 1 & Photoperiod \\
\hline PIF3 & 2 & Photoperiod \\
\hline SEP1 & 3 & Others \\
\hline SOC1 & 1 & Integrator \\
\hline SPA & 1 & Photoperiod \\
\hline TEM1 & 1 & Photoperiod \\
\hline TFL1 & 2 & Photoperiod \\
\hline TFL2 & 1 & Photoperiod \\
\hline TOC1 & 4 & Photoperiod \\
\hline VEL1 & 1 & Photoperiod \\
\hline VRN1 & 2 & Vernalization \\
\hline VRN2 & 1 & Vernalization \\
\hline WNK1 & 2 & Photoperiod/circadian clock \\
\hline
\end{tabular}

$\overline{\mathrm{z}} \mathrm{AP} 1=$ apetala1; AP2 $=$ apetala2; AGL6 = agamous-like 6; AGL8 $=$ agamous-like 8; AGL14 = agamous-like 14; AGL15 = agamous-like 15; AGL16 = agamous-like 16; AGL17 = agamous-like 17; AGL18 = agamous-like 18; AGL19 = agamous-like 19; CAL = cauliflower; identified genes were involved in various flowering pathways (Table 6; Supplemental Tables 2 and 3), including photoperiod/ circadian clock, GA, autonomous, vernalization, and aging pathways (Amasino and Michaels, 2010; Srikanth and Schmid, 2011). These include orthologs of the autonomous pathway genes such as flowering locus $\mathrm{D}$ (FLD), flowering locus with $\mathrm{kh}$ domains (FLK), and luminidependens (LD), orthologs of the GA pathway gene such as flowering-promoting factor (FPF) and orthologs of the vernalization pathway genes such as vernalization 1 (VRN1), embryonic flower1 (EMF1), and enhancer of AG-4 2 (HUA2), as well as a number of genes responding to the photoperiod pathway, such as agamous-like 15 (AGL15), CO, CCA1, CHS, CRY2, PHYA, PIF3, ELF3, LHY, TOC1, and terminal flower1 (TFL1). In addition, homologs of the key genes involved in flowering regulation, such as flowering locus C (FLC), flowering locus T (FT), CO, and suppressor of overexpression of co1 (SOC1), were also identified in this study. In addition, we identified several orthologs of the functional genes associated with floral meristem identity and flower development such as apetala1 (AP1) and sepallata1 (SEP1). There was a very high degree of similarity in the known genetic flowering pathways and a lot of critical flowering genes between I. indigotica and A. thaliana.

QRT-PCR ANALYSIS. To evaluate the reliability of RNA-Seq analysis, eight bolting and flowering-related genes, AGL15 (TRINITY_DN18713_c0_g1), AGL 17 (TRINITY_DN59446_c0_g1), ELF6 (TRINITY_DN69071_c0_g2), ELF8 (TRINITY_DN69534_c0_g2), EMF2 (TRINITY_DN66245_c2_g3), FLC (TRINITY_DN69832_c1_g1), photoperiod independent early flowering1 (PIE1) (TRINITY_DN67850_c $0_{-}$g2), and TFL 1 (TRINITY_DN21486_c0_g1), were selected for verification in five tissues (roots, stems, leaves, flowers, and fruit) using qRT-PCR (Fig. 9). The primer sequences, RNA-Seq results, and qRTPCR values are listed in Supplemental Table 4. The expression profiles of the eight genes revealed by qRT-PCR were generally consistent with the corresponding FPKM values derived from RNA-seq. The expression patterns of five genes (Fig. 9A, B, D, $\mathrm{G}$, and $\mathrm{H}$ ) fit well with the RNA-Seq results, whereas three genes (Fig. 9C, E, and F) had similar expression patterns, but

Table 6. Continued.

CCA1 $=$ circadian clock associated $1 ; \mathrm{CO}=$ constant COL $=$ constantlike; $\mathrm{CHS}=$ chalcone synthase; $\mathrm{CRY} 1=$ cryptochrome $1 ; \mathrm{CRY} 2=$ cryptochrome 2; EBS = 24-epibrassinolide; ELF3 = early flowering 3; ELF6 = early flowering 6; ELF8 = early flowering 8; ELF9 = early flowering 9; EMF1 = embryonic flower1; EMF2 = embryonic flower2; ESD4 = early in short days 4; FD = flowering locus D; FKF1 = flavinbinding kelch domain $\mathrm{F}$ box 1 ; FLC = flowering locus $\mathrm{C}$; FLD = flowering locus D; FLK = flowering locus with kh domains; FPA = flowering time control protein a; FPF = flowering-promoting factor; $\mathrm{FT}=$ flowering locus T; GA = gibberellin; GI = gigantea; HUA2 = enhancer of AG-4 2; LD = luminidependens; LHY = late elongated hypocotyl; LKP2 = lov kelch protein 2; PHYA = phytochrome A; PHYB = phytochrome $\mathrm{B}$; PHYD = phytochrome D; PHYE = phytochrome E; PIE1 = photoperiod independent early flowering 1 ; PIF3 = phytochrome interacting factor 3 ; SEP1 = sepallata 1 SOC1 = suppressor of overexpression of co1; SPA = suppressor of phya-105; TEM = tempranillo; TEL1 = terminal flower1; TEL2 = terminal flower2; TFL1 = terminal flower1; TFL2 = terminal flower 2 ; TOC1 = timing of $\mathrm{cab}$ expression 1 ; VEL1 = vernalization 5 ; VRN1 = vernalization 1; VRN2 = vernalization $2 ; \mathrm{WNK} 1=$ with no lysine kinase 1. 
TRINITY_DN66245_c2_g3
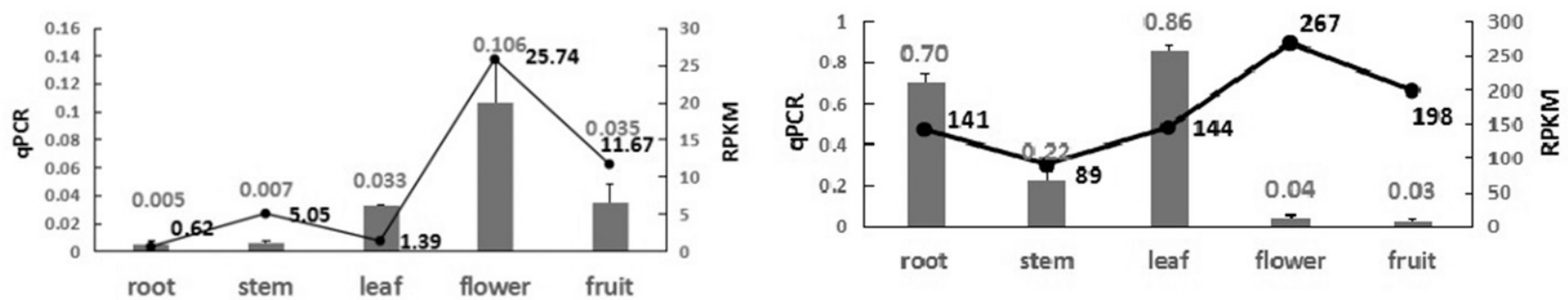

TRINITY_DN59446_c0_g1

TRINITY_DN67850_c0_g2 Q
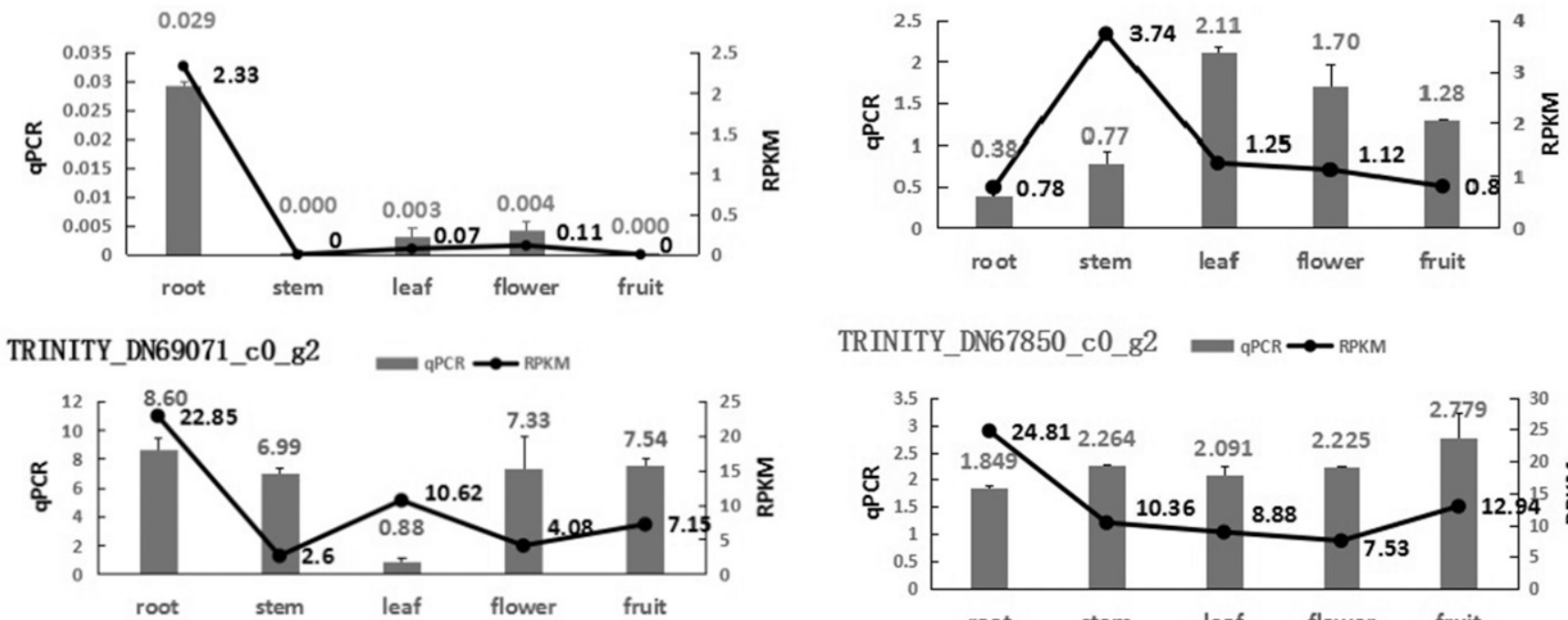

TRINITY_DN67850_c0_g2
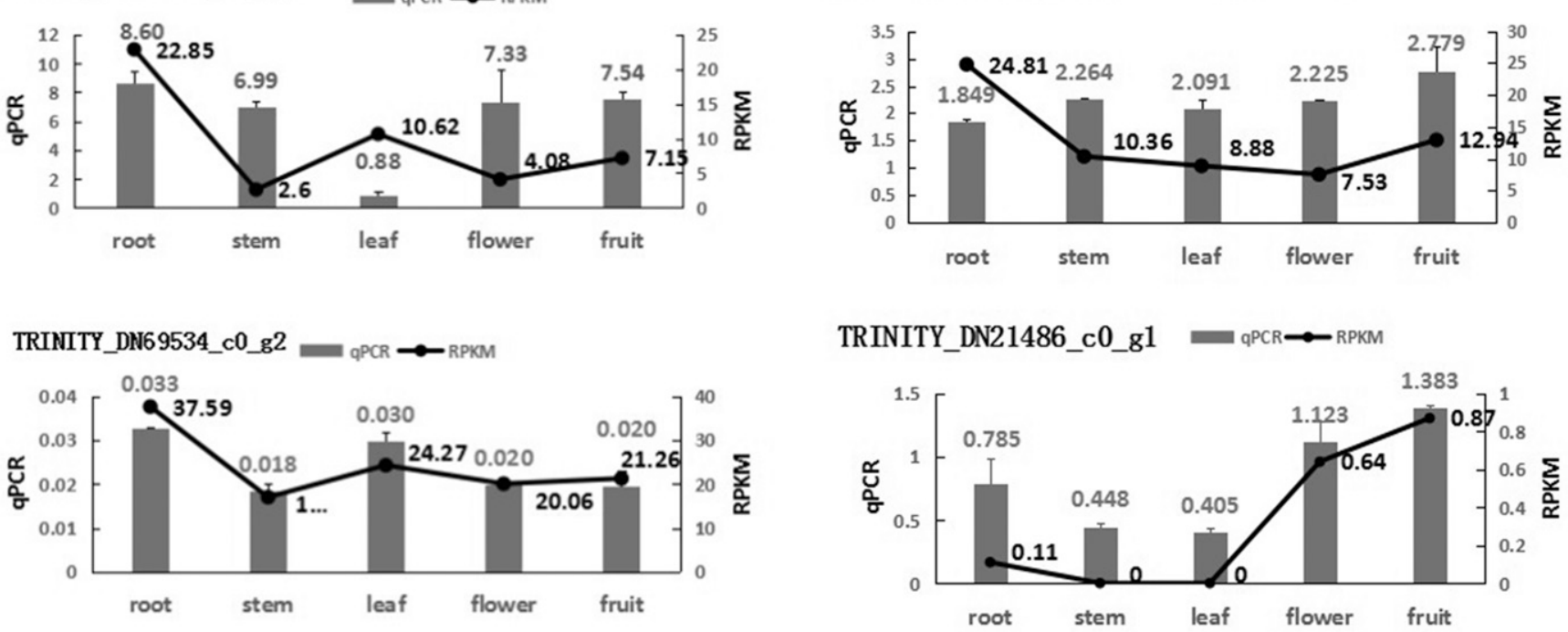

Fig. 9. Quantitative real time polymerase chain reaction (qRT-PCR) validation of eight putative genes involved in flowering pathways; RPKM= reads per kilobase of transcript per million mapped reads.

with very small inconsistencies compared with the RNA-Seq results. These results support the reliability of the RNA-Seq data.

\section{Discussion}

This study catalogs the occurrence of genes in I. indigotica that have sequence similarity to flowering gene $A$. thaliana and provides preliminary investigations of flowering-associated genes in I. indigotica. Furthermore, we compared transcriptomes of leaves, stems, flowers, and fruit with that of roots at the reproductive stages. Functional enrichment of the DEGs provided clues about the molecular basis of reproduction in I. indigotica.

I. indigotica is an important biennial herbaceous plant, and its dried leaves and roots have commonly been used as Chinese medicines for hundreds of years. Because of the low number of available $I$. indigotica gene sequences in public databases, little functional investigation has been done for this plant. Tang et al. generated 28 million Illumina paired-end reads that were then assembled into 33,238 unigenes in a previous study (Tang et al., 2014). In this study, RNA-seq and differential gene expression profiling analyses were performed. About 150 million clean reads were generated and assembled into 124,508 unigenes. The numbers of high-quality reads and assembled unigenes exceeded the previous study. The unigenes assembled in this study were subjected to a BLASTx similarity search and annotation against the nr, SwissProt, COG, KEGG, GO, and Pfam databases. The most frequent GO term in the molecular function, biological process, and cellular component groups 
were the cell part, metabolic process, and binding, respectively. These findings differ somewhat from the results in our previous study of the transcriptome of I. indigotica. The COG functional annotation of the unigenes revealed that the category "general function prediction only" was the most highly represented category. A large number of unigenes were also annotated to the category "replication, recombination, and repair," which were similar to the previous study of the transcriptome of $I$. indigotica (Tang et al., 2014). These findings establish the basis for further genomic studies in I. indigotica.

Studies of $A$. thaliana flowering have revealed that multiple different flowering pathways converge at several flowering pathway integrators such as SOC1 and FT, which were regulated by two key upstream genes, $\mathrm{CO}$ and FLC that negatively control flowering (Lee and Lee, 2010; Moon et al., 2005; Srikanth and Schmid, 2011). In this study, two unique sequences, TRINITY_DN63019_c0_g1 and TRINITY_DN63019_c0_g2, were found to be the orthologs of AtCO. Moreover, some studies suggested that lots of genes associated with the vernalization pathway played important roles in flowering control (Jung et al., 2012; Srikanth and Schmid, 2011). As expected, the vernalization-response genes including EMF1, VRN1, and vernalization 2 (VRN2) were also identified in this study (Table 6) and the result was consistent with the previous studies of flowering gene discovery (Gao et al., 2014; Moon et al., 2003; Zhang et al., 2011, 2013). The unigene (TRINITY_DN38410_c0_g3) matched to AtSOC1 was found as well (Supplemental Table 3). The interaction of SOC1 and AGL24, which belong to the MADS-box gene family regulates the expression of LFY and determines the flowering time of $A$. thaliana (Liu et al., 2008; Seo et al., 2009). The family of MADS-box transcription factor is a major group of regulators controlling floral development and floral organ specification in A. thaliana (Becker and Theißen, 2003; Smaczniak et al., 2012; Theißen, 2001). Several members of the MADS-box family such as AP1, AP2, and AGLs were identified in this study and probably take part in the regulation of the development and flowering of I. indigotica.

\section{Conclusion}

Illumina sequencing and de novo assembly were performed for I. indigotica at the reproductive stages. As a result, more than 44.97 Gbp of data were obtained and assembled into 124,508 unigenes with an average length of $1027 \mathrm{bp}$, representing orthologs of known plant genes, as well as potential new genes. The assembly and annotation of the I. indigotica transcriptome achieved in this study revealed tissue-specific gene expression patterns and pathways. This study is the first to conduct systematic identification of flowering-associated genes based on transcriptome sequencing and assembly in I. indigotica. A total of 91 unigenes that showed high homology to the known bolting and flowering-related genes in $A$. thaliana were identified. The results presented herein could build a foundation for further investigation of bolting and flowering regulatory networks in I. indigotica and contribute to molecular and genetic research in the Brassicaceae plants.

\section{Literature Cited}

Amasino, R.M. and S.D. Michaels. 2010. The timing of flowering. Plant Physiol. 154:516-520.

Bateman, A., E. Birney, L. Cerruti, R. Durbin, L. Etwiller, S.R. Eddy, S.G. Jones, K.L. Howe, M. Marshall, and E.E.L. Sonnhammer. 2002. The Pfam protein families database. Nucleic Acids Res. 30:276-280.
Becker, A. and G. Theißen. 2003. The major clades of MADS-box genes and their role in the development and evolution of flowering plants. Mol. Phylogenet. Evol. 29:464-489.

Benjamini, Y. and Y. Hochberg. 1995. Controlling the false discovery rate: A practical and powerful approach to multiple testing. J. R. Stat. Soc. B 57(1):289-300.

Boyle, E.I., S. Weng, J. Gollub, H. Jin, D. Botstein, J.M. Cherry, and G. Sherlock. 2004. GO:TermFinder - Open source software for accessing gene ontology information and finding significantly enriched gene ontology terms associated with a list of genes. Bioinformatics 20:3710-3715.

Conesa, A., S. Götz, J.M. García-Gómez, J. Terol, M. Talón, and M. Robles. 2005. Blast2GO: A universal tool for annotation, visualization and analysis in functional genomics research. Bioinformatics 21:3674-3676.

Dong, Z., O. Danilevskaya, T. Abadie, C. Messina, N. Coles, and M. Cooper. 2012. A gene regulatory network model for floral transition of the shoot apex in maize and its dynamic modeling. PLoS One 7: e43450.

Dukowic-Schulze, S., A. Harris, J.H. Li, A. Sundararajan, J. Mudge, E.F. Retzel, W.P. Pawlowski, and C. Chen. 2014. Comparative transcriptomics of early meiosis in Arabidopsis and maize. J. Genet. Genomics 41:139-152.

Gao, J., Y. Zhang, C.L. Zhang, F.Y. Qi, X.P. Li, S.H. Mu, and Z.H. Peng. 2014. Characterization of the floral transcriptome of moso bamboo (Phyllostachys edulis) at different flowering developmental stages by transcriptome sequencing and RNA-seq analysis. PLoS One 9:e98910.

Grabherr, M.G., B.J. Haas, M. Yassour, J.Z. Levin, D.A. Thompson, I. Amit, X. Adiconis, L. Fan, R. Raychowdhury, Q. Zeng, Z.H. Chen, E. Mauceli, N. Hacohen, A. Gnirke, N. Rhind, F. Palma, B.W. Birren, C. Nusbaum, K. Lindblad-Toh, N. Friedman, and A. Regev. 2011. Full-length transcriptome assembly from RNA-Seq data without a reference genome. Nat. Biotechnol. 29:644-652.

Huang, L.Y., F. Zhang, F. Zhang, W.S. Wang, Y.L. Zhou, B.Y. Fu, and Z.K. Li. 2014. Comparative transcriptome sequencing of tolerant rice introgression line and its parents in response to drought stress. BMC Genomics 15:1026.

Iseli, C., C.V. Jongeneel, and P. Bucher. 1999. ESTScan: A program for detecting, evaluating, and reconstructing potential coding regions in EST sequences. Proc. Intl. Conf. Intell. Syst. Mol. Biol. 99:138-148.

Jung, C.H., C.E. Wong, M.B. Singh, and P.L. Bhalla. 2012. Comparative genomic analysis of soybean flowering genes. PLoS One 7:e38250.

Lee, J. and I. Lee. 2010. Regulation and function of SOC1, a flowering pathway integrator. J. Expt. Bot. 61:2247-2254.

Li, B. and C.N. Dewey. 2011. RSEM: Accurate transcript quantification from RNA-Seq data with or without a reference genome. BMC Bioinformatics 12:323.

Li, L., C.J. Stoeckert, and D.S. Roos. 2003. OrthoMCL: Identification of ortholog groups for eukaryotic genomes. Genome Res. 13:21782189.

Liu, C., H. Chen, H.L. Er, H.M. Soo, P.P. Kumar, J.H. Han, Y.C. Liou, and H. Yu. 2008. Direct interaction of AGL24 and SOC1 integrates flowering signals in Arabidopsis. Development 135:1481-1491.

Livak, K.J. and T.D. Schmittgen. 2001. Analysis of relative gene expression data using real-time quantitative PCR and the $2^{-\Delta \Delta C T}$ method. Methods 25:402-408.

Mao, X.Z., T. Cai, J.G. Olyarchuk, and L.P. Wei. 2005. Automated genome annotation and pathway identification using the KEGG orthology (KO) as a controlled vocabulary. Bioinformatics 21:3787-3793.

Moon, J., H. Lee, M. Kim, and I. Lee. 2005. Analysis of flowering pathway integrators in arabidopsis. Plant Cell Physiol. 46:292-299.

Moon, Y.H., L.J. Chen, R.L. Pan, H.S. Chang, T. Zhu, D.M. Maffeo, and Z.R. Sung. 2003. EMF genes maintain vegetative development by repressing the flower program in Arabidopsis. Plant Cell 15:681693.

Mortazavi, A., B.A. Williams, K. McCue, L. Schaeffer, and B. Wold. 2008. Mapping and quantifying mammalian transcriptomes by RNASeq. Nat. Methods 5:621-628. 
Mouhu, K., T. Hytönen, K. Folta, M. Rantanen, L. Paulin, P. Auvinen, and P. Elomaa. 2009. Identification of flowering genes in strawberry, a perennial SD plant. BMC Plant Biol. 9:122.

Natale, D.A., U.T. Shankavaram, M.Y. Galperin, Y.I. Wolf, L. Aravind, and E.V. Koonin. 2000. Towards understanding the first genome sequence of a crenarchaeon by genome annotation using clusters of orthologous groups of proteins (COGs). Genome Biol. 1(5):research0009.1-0009.19.

National Pharmacopoeia Committee. 2015. Pharmacopoeia of the People's Republic of China. China Med. Sci. Technol. Press, Beijing, China.

Nie, S.S., C. Li, L. Xu, Y. Wang, D.Q. Huang, E.M. Muleke, X.C. Sun, Y.Xie, and L.W. Liu. 2016. De novo transcriptome analysis in radish (Raphanus sativus L.) and identification of critical genes involved in bolting and flowering. BMC Genomics 17:389.

Parcy, M. 2005. Flowering: A time for integration. Intl. J. Dev. Biol. 49:585-593.

Platel, R.K. and M. Jain. 2012. NGS QC toolkit: A toolkit for quality control of next generation sequencing data. PLoS One 7:e30619.

Polashock, J.J., R. Arora, Y. Peng, D. Naik, and L.J. Rowland. 2010. Functional identification of a C-repeat binding factor transcriptional activator from blueberry associated with cold acclimation and freezing tolerance. J. Amer. Soc. Hort. Sci. 135:40-48.

Sánchez-Sevilla, J.F., E. Cruz-Rus, V. Valpuesta, M.A. Botella, and I. Amaya. 2014. Deciphering gamma-decalactone biosynthesis in strawberry fruit using a combination of genetic mapping, RNA-Seq and eQTL analyses. BMC Genomics 15:218.

Sato, A., H. Okubo, and K. Saitou. 2006. Increase in the expression of an alpha-amylase gene and sugar accumulation induced during cold period reflects shoot elongation in hyacinth bulbs. J. Amer. Soc. Hort. Sci. 131:185-191.

Seo, E., H. Lee, J. Jeon, H. Park, J. Kim, Y.S. Noh, and I. Li. 2009. Crosstalk between cold response and flowering in Arabidopsis is mediated through the flowering time gene SOC1 and its upstream negative regulator FLC. Plant Cell 21:3185-3197.

Shrestha, R., J. Gómez-Ariza, V. Brambilla, and F. Fornara. 2014. Molecular control of seasonal flowering in rice, arabidopsis and temperate cereals. Ann. Bot. 114:1445-1458.

Smaczniak, C., R.G.H. Immink, G.C. Angenent, and K. Kaufmann. 2012. Developmental and evolutionary diversity of plant MADS-domain factors: Insights from recent studies. Development 139:30813098.

Srikanth, A. and M. Schmid. 2011. Regulation of flowering time: All roads lead to Rome. Cell. Mol. Life Sci. 68:2013-2037.

Stamm, M.D., L.S. Enders, T.J. Donze-Reiner, F.P. Baxendale, B.D. Siegfried, and T.M. Heng-Moss. 2014. Transcriptioal response of soybean to thiamethoxam seed treatment in the presence and absence of drought stress. BMC Genomics 15:1055.

Strickler, S.R., A. Bombarely, and L.A. Mueller. 2012. Designing a transcriptome next-generation sequencing project for a nonmodel plant species. Amer. J. Bot. 99:257-266.

Tang, X.Q., Y.H. Xiao, T.T. Lv, F.Q. Wang, Q.H. Zhu, T.Q. Zheng, and J. Yang. 2014. High-throughput sequencing and de novo assembly of the Isatis indigotica transcriptome. PLoS One 9: e102963.

Thakare, D., R. Yang, J.G. Steffen, J.P. Zhan, D.F. Wang, R.M. Clark, X.F. Wang, and R. Yadegari. 2014. RNA-Seq analysis of lasercapture microdissected cells of the developing central starchy endosperm of maize. Genom. Data 2:242-245.

Theißen, G. 2001. Development of floral organ identity, stories from the MADS house. Curr. Opin. Plant Biol. 4:75-85.

Tsuji, H., K.I. Taoka, and K. Shimamoto. 2011. Regulation of flowering in rice: Two florigen genes, a complex gene network, and natural variation. Curr. Opin. Plant Biol. 14:45-52.

Wang, J.W. 2014. Regulation of flowering time by the miR156mediated age pathway. J. Expt. Bot. 65:4723-4730.

Wang, L., Z. Feng, X. Wang, X. Wang, and X. Zhang. 2010. DEGseq: An $R$ package for identifying differentially expressed genes from RNA-seq data. Bioinformatics 26:136-138.

Wang, Z., M. Gerstein, and M. Snyder. 2009. RNA-Seq: A revolutionary tool for transcriptomics. Nat. Rev. Genet. 10:57-63.

Wixon, J. and D. Kell. 2000. The Kyoto Encyclopedia of Genes and Genomes-KEGG. Yeast 17:48-55.

Ye, J., L. Fang, H. Zheng, Y. Zhang, J. Chen, Z. Zhang, J. Wang, S.T. Li, R.Q. Li, L. Bolund, and J. Wang. 2006. WEGO: A web tool for plotting GO annotations. Nucleic Acids Res. 34:W293-W297.

Young, M.D., M.J. Wakefield, G.K. Smyth, and A. Oshlack. 2010. Gene ontology analysis for RNA-seq: Accounting for selection bias. Genome Biol. 11:R14.

Zhang, J.X., K.L. Wu, S.J. Zeng, J.A. Teixeira da Silva, X.L. Zhao, C. E. Tian, H.Q. Xia, and J. Duan. 2013. Transcriptome analysis of Cymbidium sinense and its application to the identification of genes associated with floral development. BMC Genomics 14:279.

Zhang, J.Z., X.Y. Ai, L.M. Sun, D.L. Zhang, W.W. Guo, X.X. Deng, and C.G. Hu. 2011. Transcriptome profile analysis of flowering molecular processes of early flowering trifoliate orange mutant and the wild-type [Poncirus trifoliate (L.) Raf.] by massively parallel signature sequencing. BMC Genomics 12:63.

Zhu, Q.H., S. Stephen, K. Kazan, G. Jin, L.J. Fan, J. Taylor, E.S. Dennis, C.A. Helliwell, and M.B. Wang. 2013. Characterization of the defense transcriptome responsive to Fusarium oxysporum-infection in Arabidopsis using RNA-seq. Gene 512:259-266. 


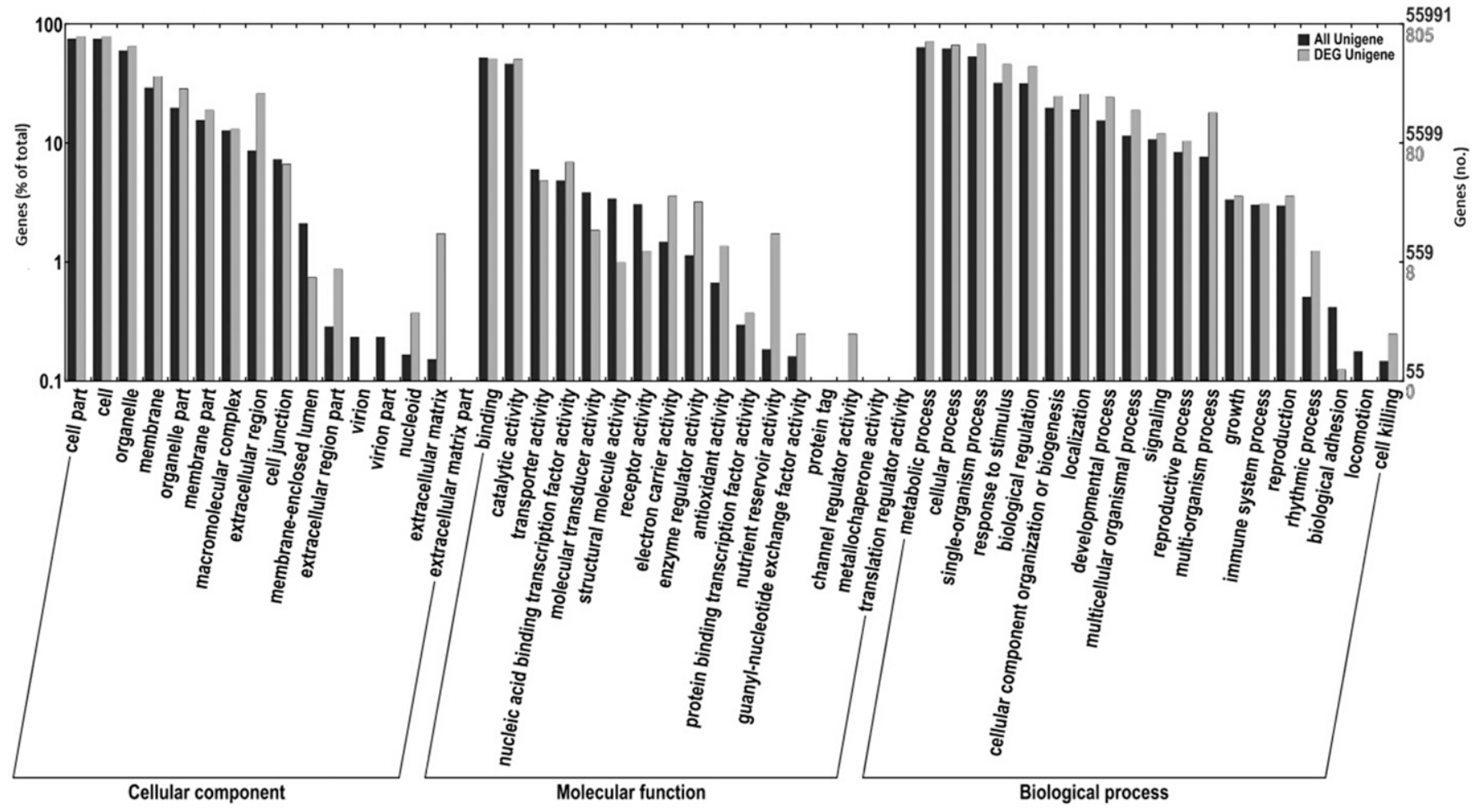

Supplemental Fig. 1. Gene ontology [GO (Boyle et al., 2004)] classification of differentially expressed genes (DEGs) in Fruit_vs._Root. 


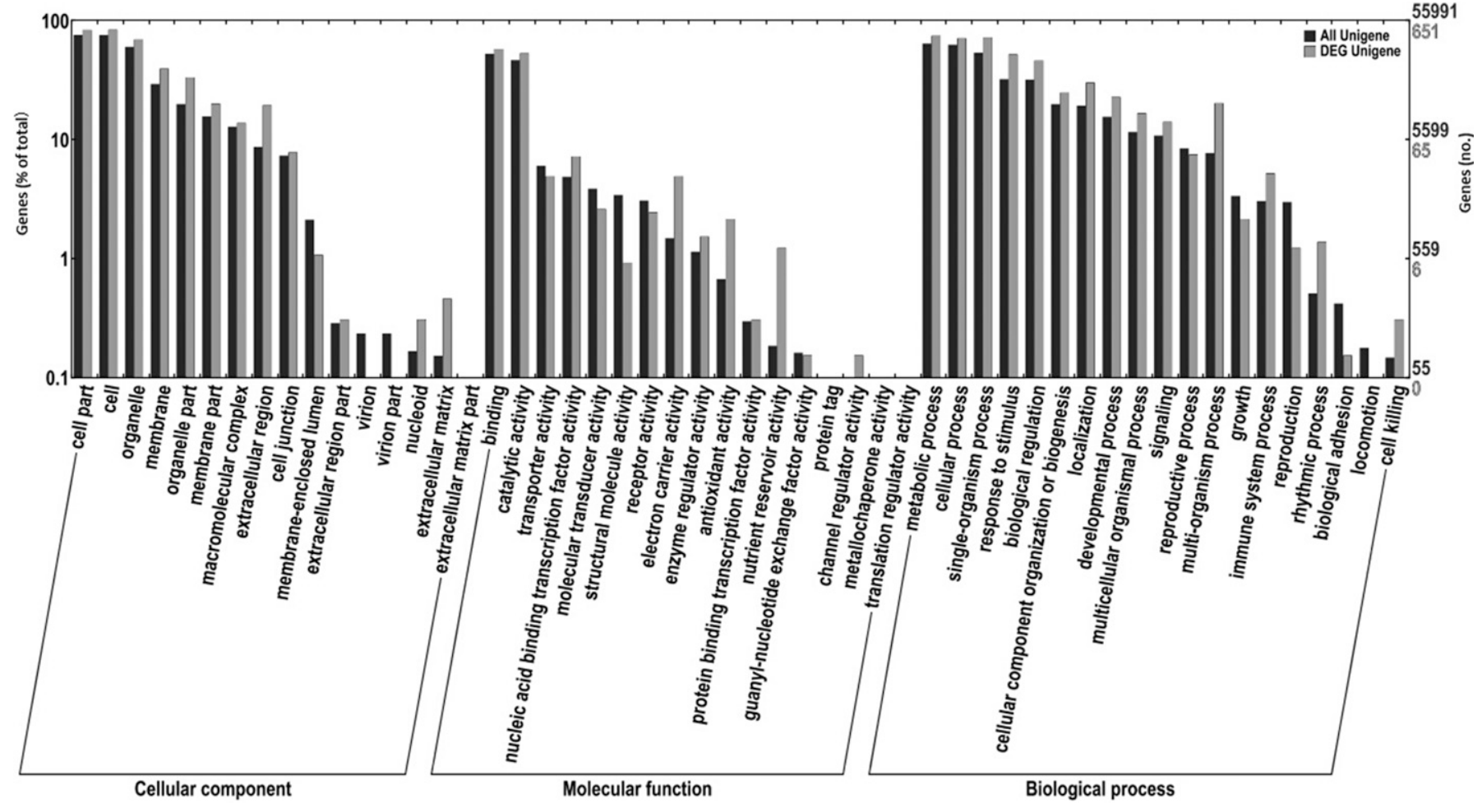

Supplemental Fig. 2. Gene ontology [GO (Boyle et al., 2004)] classification of differentially expressed genes (DEGs) in Leaf_vs._Root. 


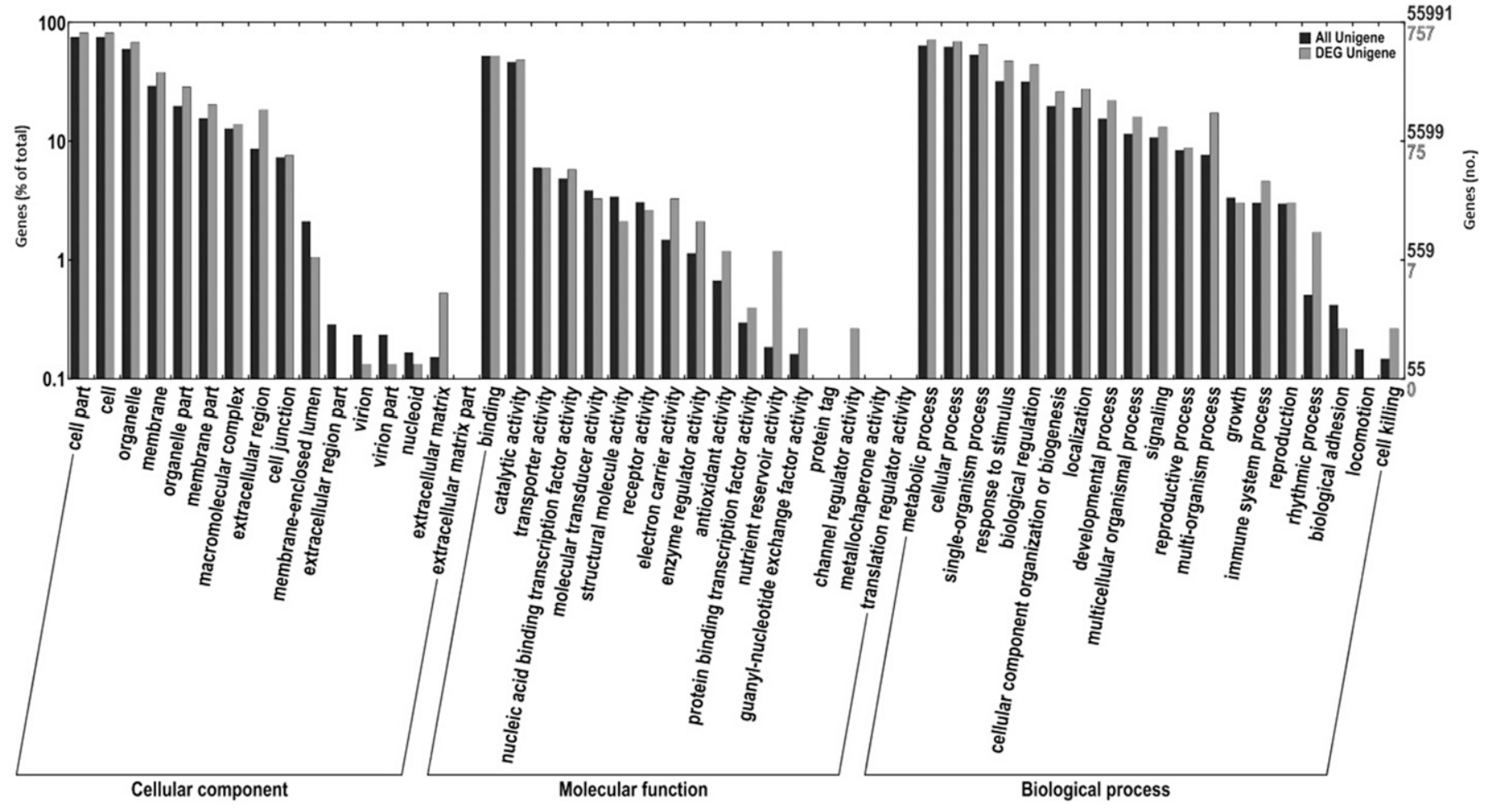

Supplemental Fig. 3. Gene ontology [GO (Boyle et al., 2004)] classification of differentially expressed genes (DEGs) in Root_vs._Stem. 
Supplemental Table 1. Kyoto Encyclopedia of Genes and Genomes [KEGG (Wixon et al., 2000)] pathways of the assembled unigenes in Isatis indigotica.

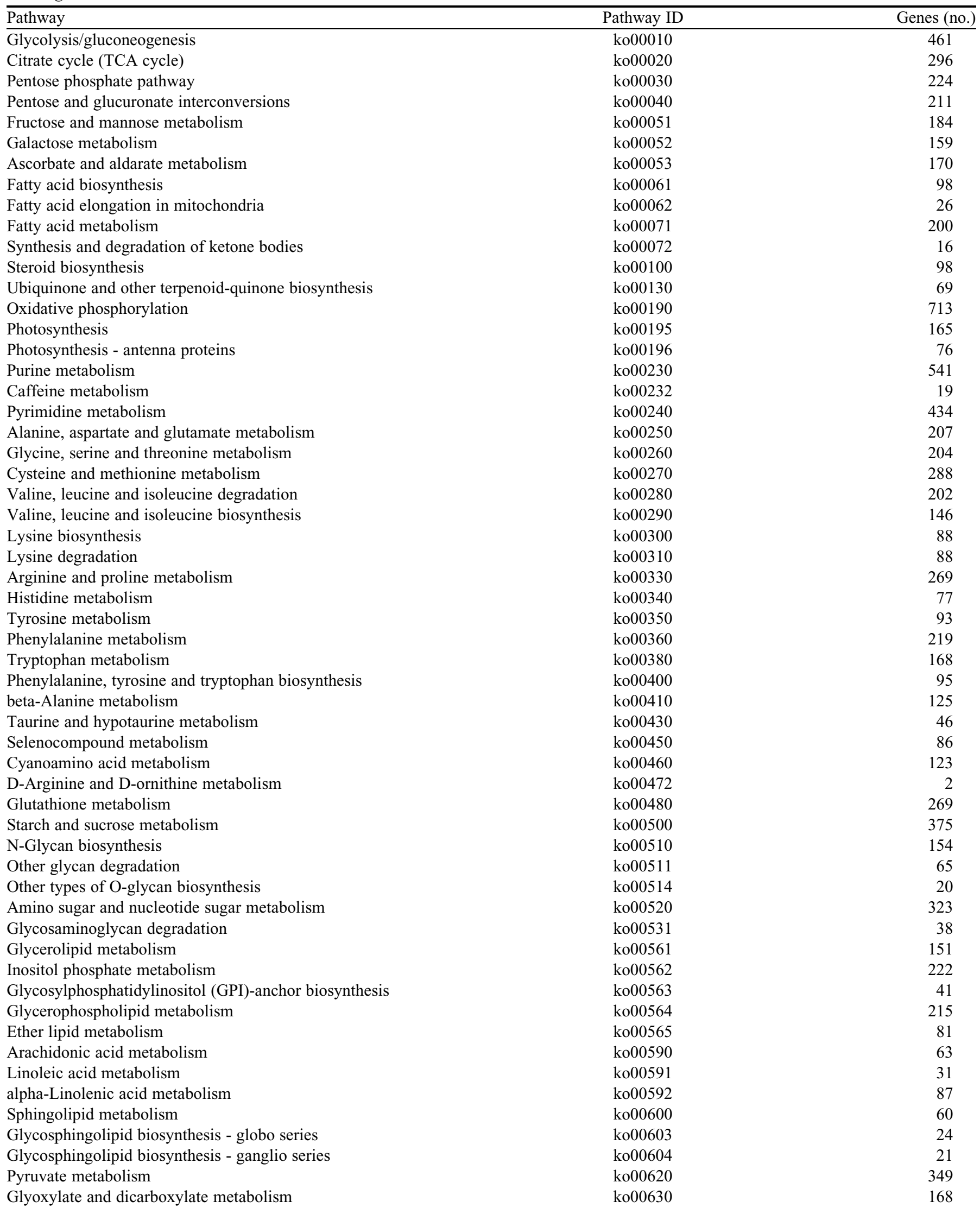


Supplemental Table 1. Continued.

\begin{tabular}{|c|c|c|}
\hline Pathway & Pathway ID & Genes (no.) \\
\hline Propanoate metabolism & ko00640 & 166 \\
\hline Butanoate metabolism & ko00650 & 95 \\
\hline C5-Branched dibasic acid metabolism & ko00660 & 19 \\
\hline Carbon fixation in photosynthetic organisms & ko00710 & 353 \\
\hline Thiamine metabolism & ko00730 & 26 \\
\hline Riboflavin metabolism & ko00740 & 28 \\
\hline Nicotinate and nicotinamide metabolism & ko00760 & 37 \\
\hline Pantothenate and CoA biosynthesis & ko00770 & 64 \\
\hline Biotin metabolism & ko00780 & 9 \\
\hline Lipoic acid metabolism & ko00785 & 13 \\
\hline Folate biosynthesis & ko00790 & 58 \\
\hline Porphyrin and chlorophyll metabolism & ko00860 & 149 \\
\hline Diterpenoid biosynthesis & ko00904 & 26 \\
\hline Brassinosteroid biosynthesis & ko00905 & 13 \\
\hline Carotenoid biosynthesis & ko00906 & 68 \\
\hline Zeatin biosynthesis & ko00908 & 40 \\
\hline Sesquiterpenoid biosynthesis & ko00909 & 17 \\
\hline Nitrogen metabolism & ko00910 & 168 \\
\hline Sulfur metabolism & ko00920 & 106 \\
\hline Phenylpropanoid biosynthesis & ko00940 & 273 \\
\hline Flavonoid biosynthesis & ko00941 & 62 \\
\hline Anthocyanin biosynthesis & ko00942 & 1 \\
\hline Flavone and flavonol biosynthesis & ko00944 & 18 \\
\hline $\mathrm{ABC}$ transporters & ko02010 & 38 \\
\hline Ribosome biogenesis in eukaryotes & ko03008 & 369 \\
\hline Ribosome & ko03010 & 1333 \\
\hline RNA transport & ko03013 & 637 \\
\hline mRNA surveillance pathway & ko03015 & 413 \\
\hline RNA degradation & ko03018 & 312 \\
\hline RNA polymerase & ko03020 & 145 \\
\hline Basal transcription factors & ko03022 & 167 \\
\hline DNA replication & ko03030 & 140 \\
\hline Spliceosome & ko03040 & 709 \\
\hline Proteasome & ko03050 & 251 \\
\hline Protein export & ko03060 & 136 \\
\hline Base excision repair & ko03410 & 109 \\
\hline Nucleotide excision repair & ko03420 & 190 \\
\hline Mismatch repair & ko03430 & 83 \\
\hline Homologous recombination & ko03440 & 96 \\
\hline Non-homologous end-joining & ko03450 & 40 \\
\hline Phosphatidylinositol signaling system & ko04070 & 222 \\
\hline Plant hormone signal transduction & ko04075 & 520 \\
\hline Ubiquitin mediated proteolysis & ko04120 & 430 \\
\hline Sulfur relay system & ko04122 & 40 \\
\hline
\end{tabular}


Supplemental Table 1. Continued.

Pathway

Pathway ID

Genes (no.)

SNARE interactions in vesicular transport

ko04130

ko04140

108

Regulation of autophagy

ko04141

81

Protein processing in endoplasmic reticulum

ko04144

657

Endocytosis

ko04145

339

Phagosome

ko04146

372

Peroxisome

ko04626

306

Plant-pathogen interaction

ko04650

441

Natural killer cell mediated cytotoxicity

ko04710

76

Circadian rhythm - mammal

ko04712 
Supplemental Table 2. The coding sequence (CDS) of flowering-associated genes in Isatis indigotica.

$\begin{array}{ll}\text { Gene ID } & \text { Coding sequence }\end{array}$

TRINITY_DN44529_c0_g2

ATGGGAAGGGGTAGGGTTGAAATGAAGAGGATAGAGAACAAGATCAATAGACAAGTGACAT TCTCTAAAAGAAGAGCTGGCCTTTTGAAGAAAGCCCATGAGATCTCGGTTCTTTGTGATGC CGAGGTATCCCTTATTGTCTTCTCCCATAAGGGGAAACTG

TRINITY_DN59583_c0_g1 ATGAGACGTACGAAGTACTTTTCAAGTTCAAATATTTTCAATCCAAAAGACAACCTTAAAAC TAAACGGACAAAGCTCATCTACGTACAGGCTGATGATCTGATTATATCAATCAATAGACT ATTTGAAAAAACAGTGCTAATATTTTCTATCTATATTTTAATTATAATGGTGCCGAAGCTA AGTGGAGATATGGAGATCTCG

TRINITY_DN60394_c3_g1

ATGTTTTTTATTATGGATGATTATAAAAATATATATGGATCTTCATGCGGCAAAGCAGCCAAG GTTGCAGTTGTAGACGGGTTCAAGGGACAGATCAAGGTCGTTCCTCCTCATTGCCATTGGA TCTTCTTCTTGATACAGGCCACCCATGTTGAGAAAAGGAGATGGCTGATGAGAGAGCATG TATGGATGCTGCATTTGATGCTGCTGCGGGGGCGGAGGGGGAGGCATGTTTTGGCCATGG TTCTGCTGGTCCCATTGCTCTTGTTGTGCCCTAAGAACCTTTTCCCTCTCCTTGATCTGTTTG GAAAGCATGCTGTTTTGTTCCTGTATGGCTTTCTCCTTTCTTTGGAGCTCATTGATGGAGTC GTACATCAGTTGGTTTTTCCTAGAGCGGATATGCTTAAGAGCAGTGTCAAGCTGCTGCTCC AGATTCTGGAGCTCCTTAGGGCTCATTGCTTGCAAGTCTTCCCCAAGATAGTGCCTCTGGT TTCTCTCCAAAAGCTCAATCTTAGCCTTAAGCCTGTTATACTCCATCGACCAGTTCGTATTG ACGTCGGACTCAGGTGCAATAAGCTGTCTCTCGGCGTAAGAGTACCTCTCATAGCGTTCAA GTATCTTCTCCATACAAGAATCAGTGGAGTATTCGAAGAGTTTCCCCTTATGGGAGAAGAC AACAAGGGCAACTTCAGCATCACAGAGAACAGAAATCTCATGAGCTTTCTTCAAAAGACC AGCTCTTCTTTTCGAGAATGTCACCTGTCTATTGATCTTGTTCTCTATCCT

TRINITY_DN64890_c0_g1

ATGTGGGATCTAAACGACTCACCACACCAGACACTTAGAGAAGAAGAATCTGAAGAGTTCTG TTATTCATCACCGGGTAAACGGGTCGGATCTTTCTCGAATTCAAGTTCTTCCGCTGTAGTC ATCGAAGATGGATCCGATGAGGACGAACATAACCGGGTCAGACCCAACAACCCACTTGTC ACACATCAGTTCTTCCCGGAGATGGAATCTAGCGTCGGAGACGGCGGCGTAGGAGATGAT GGTGGTGGTCCCGGGTCGGGGTTTCCTCGGGCTCACTGGTTTGGTGTGAAGTTTTGTCAGT CCGATCTAGCCACCGGATCATCGGCGGGTAAACCCACCACTGTCGCCGCCGTGGTCGAGC CGGTGCAGCCTTTGAAAAAGAGCCGGCGTGGACCACGGTCACGAAGTTCTCAGTATAGAG GCGTTACGTTTTACCGGCGAACCGGAAGATGGGAATCTCATATCTGGGACTGTGGGAAAC AGGTTTACTTAGGTGGATTTGACACTGCTCATGCAGCTGCTCGAGCATATGATAGAGCTGC TATTAAATTCCGTGGAGTAGAAGCTGATATCAATTTTAACATCGAAGATTATGATGATGAC TTGAAGCAGATGACGAATTTAACCAAGGAAGAGTTCGTGCACGTACTTCGCCGACAAAGC ACAGGCTTCCCTCGAGGAAGTTCGAAGTATAGAGGTGTCACTTTGCATAAGTGTGGTCGT TGGGAAGCTCGAATGGGTCAATTCTTAGGCAAAAAGTATGTTTATTTGGGGTTGTTCGACA CCGAAGTTGAAGCTGCTAGAGCTTATGATAAAGCTGCAATCAAATGTAATGGCAAAGACG CTGTGACTAACTTTGATCCAAGCATATACGACGACGAACTGAATGCCGAGTCATCAGGGA ATCCTATTCAACAAGATCATAATCTCGATTTGAGTTTGGGAAATTCGGTTAATTCGAAGCA AAAGGGTCAAGATATGCGGCTCAAGATGAACCAACAAGATTCTCTTCACTCTAATGAGAT TCTTGGATTAGGTCAAACCGGAATGGTTAACCATATCCCCAATTCAAATCACCAA

TRINITY_DN62832_c0_g1

ATGAGCGTGAAGGAACTGCAAGCGTTGGAGAGGCAGCTGGAAGCCGCTCTTACCGCAACTC GACAACGCAAGACTCAAGTTATGATGGAAGAAATGGAAGATCTTCGGAAAAAGGAGAGG CAACTCGGAGACATAAACAAACAACTCAAGATTAAGTTTGAGACCGAAGGCTATGCGTTC AAAACGTTTCAAGACTTATGGTCAAATTCGGCAGCATCGGTGGCCGGTGATCCTAACAATT CTGAATTTCCGGTTCAGTCTTCTCATCTTCAATCAGTGGATTGCAACACTGAACCCTTTTTA CAAATAGGGTTTCAACAAGATTACTACGTGCAAGGTGAAGGGTCTTCGGTATCAAAGAGT AACGTGGCATGTTCGACCAATTTCGTTCAAAATTGGGTTCTT

TRINITY_DN48318_c3_g1

ATGGGAAGGGGTAGGGTTCAGCTGAAGAGGATAGAGAACAAGATTAATAGACAAGTTAC TTTCTCAAAGAGAAGGTCTGGTTTGCTCAAGAAAGCTCATGAGATCTCTGTTCTCTGCGAT GCTGAGGTTGCTCTCATCGTCTTCTCTTCCAAAGGCAAACTCTTCGAATATTCCACCGACTC TTGCATGGAAAGGATACTTGAACGCTATGATCGCTATTTGTATTCAGACAAACAACTTGTT GGCCGAGATATTTCACAGAGTGAAAATTGGGTTCTAGAACATGCTAAGCTCAAGGCGAGA GTTGAGGTACTTGAGAAGAACAAAAGGAATTTTATGGGGGAAGACCTTGATTCGTTGAGC TTAAAGGACCTTCAGAGCTTGGAGCATCAGCTCGATGCTGCTATCAAGAGCATTAGGTCA AGAAAGAACCAAGCTATGTTTGAATCCATATCAGCGCTCCAGAAGAAGGATAAGGCTTTG CAGGATCACAATAATACGCTTCTCAAAAAGATTAAGGAGAGGGAGAAGAAAACGGGTCT GCAAGAAGGACAATTAGTCCAATGCTCCAACAATTCATCAATTCTTCAACCCCAATACTG CTTAACTTCCTCCAGAGATGGCTTTGTGGGGAGAGTTGGGGGCGAAGACGGCGGTGCATC GTCGTTGACGGAACCAAACTCTCTTCTTCCAGCTTGGATGTTACGTCCTACAACGAACGAG 
Supplemental Table 2. Continued.

\begin{tabular}{lr}
\hline Gene ID & Coding sequence \\
\hline TRINITY_DN68423_c2 g1 & ATGATCAAAGCAACTTCAGCATCACAAAGGACAGATAA
\end{tabular}

TRINITY_DN68423_c2_g1

TRINITY_DN18713_c0_g1

TRINITY_DN69985_c0_g2

TRINITY_DN59446_c0_g1

TRINITY_DN58069_c0_g1

TRINITY_DN68423_c2_g2

TRINITY_DN46328_c0_g1
ATGATCAAAGCAACTTCAGCATCACAAAGGACAGATAACTCGAAAGCTTTCTTCAAAAGTCC ATTTCTTCTCTTGGAGAAGGTCACTTGCCTGCTCGTTGCGTTTTCTATCCTCTTCATCTCGG TCTTTCCTCTCACCATCCCTCTTGATTTGTATTGTGTATGCGCTTTGGATGGATTGATGAGT TTATCTTTTTGTGTTTATATCTATCTGTGTGGGTGGAGA

ATGGGTCGAGGAAAAATCGAGATAAAGAGGATCGAGAATGCGAATAGCAGGCAAGTTACTT TCTCCAAGAGGCGTGCTGGGTTGCTCAAGAAGGCTCATGAGCTCTCTGTTCTCTGCGACGC CGAGGTTGCCGTCATCGTCTTCTCCAAGTCCGGCAAGCTCTTCGAGTTCGCTAGTACTGGC ATGAAGAAGACGCTTTTAAGATACGGAAACTACCAGAGTTCTTCAGATGCTCCTCTGACTA ATTCTAAAGCAGAGGACCAGAAGGATTGTAGAGAGGTGGACCTTTTAAAGGATGAGATTT CAAAGCTTCAAGAGAAACAACTACAATTGCAAGGTAAGGGCTTGAATATTCTGAGCTTGA AAGAGCTGCAACACCTTGAGCAGCAACTAAATGTTTCATTGATATCAGTGAGAGAGCGAA AGGAACTATTGTTGACTAAACAACTTGAAGAATCACGGCTCAAGGAACAGCGAGCAGAGC TGGAAAATGAGACCTTGCGTAGACAGGTTCAAGAACTCAGGAGTTTTCTCCCGTCCATCAA CCAACACTCTGTTCCATCATACATCAAATGCTTCGCTATAGATCCCAAGAACCCACTCGTA AACAACTCTAGCTTGGACGACATAAACTGCAGCCTCCAGAAGACCAATTCAGACACAACT TTGCAATTAGGGTTGCCGGGAGAAGCACATGATAGAAGGAAGAATGAAGGTAACGGAGA GAGCCCATCAAGTGATTCGGTGACAACGAGTACGACTGGAGCAGCTGCACAAAGGATCG GGCTAGTT

ATGGGAAGGGGGAAGATCGCGATCAAGAGGATCGACAACTCCACGAGCCGTCAGGTGACTT TCTCGAAGCGGAGGAACGGGTTGCTGAAGAAAGCCAAGGAGCTTGCGATTCTCTGCGATG CAGAGGTCGGTGTCATCATCTTCTCCAGCACCGGCCGCCTCTACGATTTCTCCAGCTCTAG CATGAAATCGGTAATAGAGCGATACAGCGAGGCCAAAGGTGAAACCAACTCGGAAATTA ACCCAGCTTCAGAAATCAAGTTTTGGCAAAAGGAGGCCGCGATTCTAAAGCGACAGCTAC ATAACTTGCAAGAAAACCACCGGTATGTTTTGCATCCTCTGAGTTCGTCAAACACTGTCGA TATTAACAAACTAGAACTGGGAACGATGTAC

ATGGGGAGAGGGAAGATAGTGATCCAGAGGATCGATGATTCGACGAGTAGGCAAGTCACTT TCTCGAAAAGAAGAAAGGGTCTCATCAAGAAAGCTAAAGAACTCGCTATTCTCTGCGACG CAGAGGTGGGTCTCATCATCTTCTCCAATACCGACAAGCTCTATGACTTTGCCAGCTCCAG TATGAAATCGGCTATTGAACGATTTAACAAGACCAAGATGGAGCAGCAACAACTATTGAA TCCTGCGTCAGAAGTTAAGTTTTGGCAGAGAGAGGCCACAACTCTAAGGCAAGAACTGCA CTCATTGCAGGAAAGTCATCGACAACTAACAGGGGAGCAATTACATGGTCTGAGCGTTAA GGAGTTGCACAATATAGAGAGCCAACTTCAAATGAGTTTACATGGAATTCGTATGAAAAA GAGGGATCTTGTTCATCAGGAGAACCTCGAATTGTCGCAAAAGGTACAAAGGATTCATCA AGAAAACGTCGAACTACACAAGAAGGCTTATGCAGCGTCAAGCAGAAATGGGTTGGGAC TTCGTGAGCTTGAAGATACAGCCGATGAGTCCCATGCACAGGTTCGTCTGCAGCTAAGTC AGCCTGAACAGTCCCATTATCAGACCTCATCAAAGAGC

ATGAAGAAGGCTAAAGAGCTCTCGATTCTCTGCGACGCCGATGTTGCTCTTATCATCTTCTCC AGCACCGGCAAGATTTACGATTTCTCCAGCGGCTGTATGGAGCAAATCCTTTCTAGATAT GGATACAGTGCTGCGCCTGCTGATCATCATCATAAACAGAGAGAACAACAACTTCTACTT TGTTCTTCACATGAAAATGGTGATGTGTTGCGAAAAGATGATTCCTTGAAGAGCGAACTC GAGAGATTACAGCTTGCAATTGAGAGACTGAAGGGTAAGGAGCTTGACGGTATGAGCTTC TCGGATCTTATTTCTCTTGAAAACCAGTTGAATGATAGCTTGCATAGTGTCAAGGATCGTA AGACTCAAGTCCTGCTTAACCAGGTAGAAAGATCCCGGTTACAGGAGAAAAGAGCGTTGG AAGAAAATCAAATCTTGCGCAAACAGGTAGAGATGTTGGGGAGAGGTTCATCAGCGCCAA AAGGGTTGAGTGAAATACCTCAGCTTTCTAGCCCACAAGCGGAGCCTGAGAGTTCTTCATC AGACGATGACGAGAATGACAACGATGATCACCATTCCGACACTTCCTTGCAGCTGGGGTT GTCGTCATCGGGGTACTGTGCAAAGAGAAAGAAGCCGAAGATTGAATCGCCCTGCGATAA CTCTGGAAGTCAAGTAGCTTCTGAT

ATGGTGAGGGTAAAGACGGAGATGAAGAGGATAGAGAACGCAACAAGTAGGCAAGTGACCT TCTCGAAGAGAAGAAATGGACTTTTGAAGAAAGCTTTCGAGTTATCGGTCCTTTGTGATGC TGAAGTCGCTTTGATCATCTTCTTTTCAAGATCCAAGCTCTATGAGTTCTCTAGCTCTAGAG CATCCGTGAGGGCAATTATAGCTGGC

ATGAAGAGGATAGAGAACAAGATCAATAGACAAGTGACATTCTCTAAAAGAAGAGCTGGCC TTTTGAAGAAAGCCCATGAGATCTCGGTTCTTTGTGATGCCGAGGTATCCCTTATTGTCTTC TCCCATAAGGGGAAACTGTTCGAGTACTCCTCTGAATCTTGCATGGAGAAGGTACTAGAA CGCTACGAGAGGTACTCTTACGCCGATAGACAGCTGAAAGCACCTGACTCCCACGTTAAT GCACAGACGAACTGGTCAATGGAATATAGCAGGCTTAAGGCTAAGATTGAGCTTCTGGAG AGGAACCAGAGGCATTATCTGGGAGAAGATTTAGAATCAATTAGCATAAAGGAGCTCCAG

Continued next page 
Supplemental Table 2. Continued.

\begin{tabular}{lr}
\hline Gene ID & Coding sequence \\
\hline TRINITY_DN60394_c3_g2 & ATGTACGAGTCCCTCAACCACCTCCAAAGAAAGGAGAA
\end{tabular}

TRINITY_DN60394_c3_g2

TRINITY_DN68029_c0_g1

TRINITY_DN63019_c0_g1

TRINITY_DN63019_c0_g2

TRINITY_DN65596_c1_g1
ATGTACGAGTCCCTCAACCACCTCCAAAGAAAGGAGAAGGAAATACAGGAGGAAAACAGCA TGCTTGCCAAACAGATAAAGGAGAGGGAAAGTATTCTAAAGACACATCAAAACCAATGGG AGCAGCAGAACCGCACCCACCATATACCAACCCAGTCGCAACCGCAATTACATCCTTACA TGATCTCTCATCAGACATCTCCTTTCCTAAATATGGGTGGACTGTACCAAGAAGAAGATCC AGCGGCGGTGAGGAGGAACAATCTC

ATGTTGATCTTCCACTTCTGTCATTTAAACTTCTTACTAGTGTCATTGGAACCCAATAGATTTT TGTTTGTTTGCGTTTTTCTAGAGGGTAACAAGATTACTGTCATCTCTCAGGTAGAGAAAGA GGCTGAAGCTAAAGGTGTACCAATGGGCCAAGCGCTAGACATAGCTATTCCTCCTCCACG GCCTAAACGAAAACCAAGCAATCCGTATCCTAGAAAGACGGGAAGTGGAGCTATCCCCAT GTTAAAATCGGGTGTAAATGATGGAAAAGAGTCCATTGGTTCAGAAAAAGTTTCGCCTCC TGAGATGGCGAATGAAGATCTGCAGGAAGACAACTGTTCAGATTGTTTCACTCATCAACA CCTCTCTGCTGCGTCTTCCATGAATAAAAGTTCTGTAGAGACATCAAACGCAAGCACTTTT CGGGAGTTCTTACCTTCACGGGAAGAGGGGAGTCAGAATAACGGGATAAGAAAGGAGCC AAACCCAGCGAATTACAGTGGTTTGAATGCAAAACATCTGGAAAACGATGTGGTAAATAA CGAGCAAGAACCTCAGACTTATCCAAGGCGTATCCCCGTTGTAGTGCCATTGGGAAGCTC GATAACAAGCTCTCTATCACATCCTCTTTCGATGCCAGACGAGAATGACGCCCCAAGAAA TAGTTATCCCCACACAGCTCAGTCGTTTCCTGACCATATAATGTCAGCCCTTTCACAGACA CCGGCTCTATATACCGCAGCAACTTTCGCCTCATCGTTTTGGCCTCCAGATTCTGGTGGTG GACCACCTGGCCAAGGGAACTTACCTCCGAATTTGGCTGCCATGGCTGCAGCCACTGTCG CAGCTGCAAGTGCTTGGTGGGCTGCCAATGGATTGCTACCTTTATGTGCTCCCTTTAGTTC AGGTGGTTTGACCTGCCACCCTCCAACTACTTTTGGACCATCCGGTGAAGTAGACCACACA AAAGCAGGCACTTTACTACATGTTTCTGCGCAGAGCCGAGAGCGGATGGACCAAGAACAC TCTGAGGCATCAAAGGCTCGATCTTCATTGGACTCGGAGGAGATTGAAAACGGGATTAAA CCAGATTGTCATGAGCAGCCTTCAGCAACAGCTGAGACTGATGTGAAAGGCTCAGATGGA GCAAGAGACAGAAAACAAGTTGACCGGTCTTCCTGTGGTTCAAACACTCCCTCGAGTAGT GATGATGTCGAGGCGGATGCATCAGAGAGGCAAGAGAATGGCACCAATCCTGAAGTGAA AGAAGTGAATGCAGATACTACTAATCCACAAACTGCAGAGTCCAATGCACGTCGCAGTAG AATCAGCTCCAACTTAACCGATCCATGGAAGGCTGTTTCAGATGAGGGTCGAATCGCTTTC CAAGCTCTCTTTGCCAGAGAAGTTTTGCCACAAAGTTTTTCATATCAGAAAGATTACAGAG AAGAGGATCAACAAGAACATAGATATCCAATGGAACTTGATCTTAACTGCACAGCTCAAT TAGCTCCGGTTGATGATGATCAAGAGGAGAAGAGAAACATAGGGTTTTTGGGAATGGGAT TGGATGCCTCAAAGCAAAGTAGAGGAAGAACAGGTTTCAAACCTTACAAAAGATGTTCAA TGGAAGCCAAAGAAAGTAGAGTGATCAGCACCAATCCTATCATCCATGTGGAACAGAAAG ATTCCAAACGGATTCGGTTGGAAACCCAGGCCTCCACA

ATGGCCACTCACCACACAACAATGACCGATCCGGAGAACAGACTGGTGGTGGTTCAAGATG AGGAGGGTGAAGAGGATGCCAAAGAGGCGGCTTCGTGGTTGTTCCCTAACTCAGACAAA AACAATGGGAATCACCAGAACAATGAATTGTTGTTCAGTGATGAGTATCTAGACCTTGCG GATTACAACTCGAGCATGGACTACAAATTCACAGGCCAATACAATCAGTATCAGCGTCAA CAAGACTGCAGCGTACCACAGACAAGCAACGGAGGAGACAGGGTTGTTCCGCTTCGACT TGAAGAATCAAGGGGAAACCTGCGCCACAAGCAACAGAATATCACATATGGCTCCTCAGC AAGTCACTACGTGTACAATGGTTCCAAAAAACAGAACGTAAGGCTTATGTATATTTGT

ATGTTGAAACAAGAGAGTAACGACATTGGTAGTAGAGAGAACAACAACAGAGGGGCACGAG CCTGTGACACATGCCAGTCAACTGTCTGCACCGTGTACTGCCATGCTGACTCTGCCTACTT GTGCACGAGCTGTGATGCTCAAGTTCACTCTGCCAATCGCGTTGCTTCCCGCCATAAACGG GTCCGTGTCTGCGAGTCATGTGAGCGTGCCCCGGCTGCTTTTCTGTGCGAGGCAGATGATG CGTCTCTATGCACAGCCTGCGATTCAGAGGTTCATTCCGCAAACCCTCTTGCCAGACGCCA TCAGCGAGTTCCAATTCTGCCGGTTTCTGGAAACTCTTACAGCTCCATGGCCACTCACCAC ACAACA

ATGGGTTACATGTGTGACTTCTGTGGTGAACAAAGATCAATGGTGTATTGTCGATCCGATGC AGCGTGTCTCTGCCTCTCCTGTGACCGGAGTGTTCATTCCGCTAATGCATTGTCCAAACGA CACTCTAGGACACTTGTCTGCGAGAGATGCAATGCGCAGCCTGCAACAGTCAGGTGTGT TGAAGAAAGGGTTTCGCTCTGTCAAAACTGTGATTGGTCAGGCCACAACAACAATTCTTC GTCTTCTTCGTCAAATAATCACAAGAGGCAAACGATTAGTTGCTATTCTGGTTGCCCTTCA AGCTCGGAGCTCGCCTCTATCTGGTCTTTCTGTTTGGACTTAGCCGGACAATCCGTTTGTGA ACAAGAAATGGGTATGATGAATATAGACGATGATGCTCAGACCAACCAAAACTGTAACGA TGATAAGAAAGATGCCATCGTAGGCTCCTCTTCAAGGCCTGAAACCAGTTCTGCAGCAC AACCGAATTCATCACTTGCTAAGGATGTTGGAGTGTGTGAAGATGACTTTTATGGAAACCT 
Supplemental Table 2. Continued.

\begin{tabular}{ll}
\hline Gene ID & \multicolumn{1}{c}{ Coding sequence } \\
\hline CGGTATGGATGAAGTTGACTTGGCCCTTGAGAACTATGAAGAGCTCTTTGGGACAGCCTT \\
TAACCCCTCGGGAGAGCTCTTCGGACAAAGTGGAATCGATAGTCTTTTCCAGAAGCATC \\
AAGCAGCTCCTGAGGGCGGGAATTCGGTGCAGCCTGCTGGGAGCAATGATTCTTTCATGA \\
GTTCAAAAACCGAGCCAATAATATGCTTCACATCGAAGCCAGCACATTCGAACATATCT \\
TTCTCTGGAGTCACAGGAGAAAGTAGTGCTGGAGATTTCCAAGAATGTGGTGCATCAT \\
CTTCCATGCAGCTCTCGGGCGAGCCACCATGGTTTCCTCAAACATCACAGGATAATAATG \\
CTTGCTCACACTCAGTGACCCGTAATAACGCGGTTATGCGTTACAAAGAAAAAAAGAAGG \\
CTCGCAAGTTTGATAAGAGAGTGAGGTATGCTTCTCGCAAAGCAAGAGCTGATGTGAGAC \\
GGCGTGTAAAGGGGAGATTTGTCAAAGCTGGTGAAGCTTATGACTACGACCCACTCACCC \\
CAACCAGAAGCTAT
\end{tabular}

TRINITY_DN53874_c0_g1

ATGACTACGGTCATGGGTGCATTGTCGTTGGATGAGATCAGACATGCACAAAGAGCGGATGG ACCCGCAAGCATCTTGGCAATAGGCACGGCCAACCCTGCGAACTATGTGATGCAGGCGG ATTATCCAGACTACTATTTCCGCATCACCAACAGTGAACACATGACCGATCTCAAGGCAA AGTTCAAGCGCATGTGCGACAGATCAATGATACGGAAGCGGTACTGCACT

TRINITY_DN61788_c0_g1

ATGCTTTTAACTACATTCCCCTGTGAGTTTTGTGTTGCAGTGATGTCATCTCATGACTCAAT GGGGCTGGTTTTTAAGTGCTTATCAAAAGGAGCAGTTGACTTTCTCGTTAAGCCGATAA GAAAAAACGAGCTTAAAATCCTTTGGCAACATGTTTGGAGAAGATGTCAAAGTTCTAG CGGGAGTGGAAGTGAAAGTGGAACACATCAAACTCAAAAGTCCGTGAAATCAAAGAGTA TTAAAAAATCTGACAATGATTCCGGAAGCAGTGGTGAGAATGAAAACGGGAGCATTGGC CTGAATGCTAGTGATGGAAGTAGTGATGAGAGTGGTGCTCAGAGCTCTTGGACGAAAA AAGCTGTGGAGGTTGACAGTCCACGAGCGGTATCTCCATGGGATCGAGTTGATAGCACT TGCGCACAAGTGGTACATTCCAACCCCGAGGTTCCCAGTAATCACTTGGTTGCAGCACC TGCTGAGAAGGAGACCCACGAACAGGATGAAAAATTTGAAGATGTCACAAGGGGTAG AGACTTGGAGATTAGTATTCATAGAAATTGTGATCTGACACTGGAGCCAAAAGATGAACC CCTAACCAAAACCACTGGCATTATGAGACAGGATAATTCATTTGAAAAGAGCTCTAGT AAATGGAAATTGAAAGTTGGAAAAGGACCATTGGACCTCAGTAGTGAAAGCCCTTCAA GTAAACAAATGCATGAAGATGGAGGGTCGGGTTGCAAAGCTATCTCTTGCCACCTTC AAGATAACAAAGAACTCGAGGCGCCTAACACACACTGCAAAACTTCAGATACCTGTGAA GCTGCCGTCAAAAACGCTGAAGAGCTAATGGACGTTGAACATAGTTCAAAGAGGCATA GAGGAACTAAAGATGATGGGGTAATAGTTAGAGATGACCGCAATGTGCTGAGGCGTT CAGAGGGTTCAGCTTTCTCAAGGTATAATCCAGCCTCAAATAACAACAAGCTTTCTGGCG GGAACTTGGGAAGCAACTCTCTTCAAGATAATAATTGTCACTCAAACATGAACGAGAGC CTCCCCAATAATCATCACTCACGCGTCGGTAGCAATAACTTAGAAATGAGCTCCACA ACTGAGAACAACGCATTCACAAAGCCTGGAGCTCCAAAAGTAAGCTCGGCAGGATCTTCA TCAGTAAAGCATTCATCGTTTCAGCCTCTACCTTGTGATCATCATCAGTCCTTCTAT AATCTTGTTCACGCCCCTGAGCGGAAGTTACCGCCACAATGTGGATCCTCAAATGTGTAC AATGAGGCGATTGAAGCTAACAACAACACAGTGAATTACAGTGTGAATGGAAGTGTA TCAGGTAGTGGTCATGGAAGCAATGGGCCATATGGAAGCAGCAACGGCATGAATG CTGGAGGGATGAATATGGGAAGTGATAATGGTGCTGGCAAAAGCGGAAGTGGCGATGG TAGCGGAAGTGGAAGTGGAAGCGGAAGCGGGAATGTGGCAGATGAAAATAA GATCTCTCAAAGAGAAGCTGCTTTGACGAAGTTCCGCCAGAAGAGAAAAGAGAGG TGCTTCAGAAAGAAGGTACGATACCAAAGCCGGAAAAAACTAGCGGAACAACG GCCTCGTGTCCGTGGCCAATTCGTCCGTAAGACAGCTGCTGGAACAGATGATAACGACA TAAAAAACGCCGAGGATAGC

TRINITY_DN29253_c0_g1 ATGCATACACCAAAACAAAACATAACCCACAAGACTAAAGATTATTCCAAACACAAAGAT GATATCTTTTTAAGCAAACCCTTAATAAAAAAAATCAAGAGAGGGAAGGGAGATGAA CACAGACCATACAAGTGGTTGAAGAAGATC

TRINITY_DN45325_c0_g1

ATGTTTAACCGGAGTTACAGCCCTTATGAAGTTCCTCTCCTTTTGCATCTTCAGTTTTCCCTT TTTACCTATAAAACCAGACAGACCACAGCAAATTTTTCAAACATGAAAATCGATACTA CTGGGTTAACACCAGTGTAC

TRINITY_DN66742_c0_g1

ATGTCTTATTCTGGCTCTGGTTCTGGTTCTGGTGGTGGTGGTTGTAGTATTGTATGGTTTAGA AGAGATCTCAGGGTTGAAGATAATCCAGCTTTAGCTGCAGCTGTAAGAGCTGGCCCTG TAATTGCAGTGTTTGTATGGGCACCAGAGGAAGAGGGACACTATCATCCTGGTAGGGTT TCAAGGTGGTGGCTCAAGAATAGTTTGGCTCAGCTTGATTCTTCTCTTAGAAGCCTTGG TACATGTCTCATCACCAAGAGATCTACTGATAGTGTTGCTTCACTTCTTGAAGTTGTTAA ATCCACTGGTGCTTCTCAGATCTTCTTCAACCACTTGTATGATCCCTTATCATTGGTCCGT GATCATCGTGCTAAGGACGTTCTGACAGCGGAAGGCATAGCGGTTAAATCATTCAACGC

Continued next page 
Supplemental Table 2. Continued.

\begin{tabular}{ll}
\hline Gene ID & Coding sequence
\end{tabular}

AGATTTGCTTTACGAGCCATGGGAAGTGACCGATGAATTAGGCCGTCCTTTCTCAGTG TTTGCTGCCTTTTGGGAAAGATGCCTTAGCATGCCTTATGACCCTGAGTCTCC TCTTCTCCCACCTAAGAAGATCATTTCAGGGGATGTGTCCAAGTGTGTT GCGGATACATTGGTCTTTGAAGATGAATCAGAGAAAGGAAGTAATGCACTTCTCG CTCGTGCTTGGTCTCCGGGTTGGAGTAATGGTGATAAAGCTCTCACAACGTTCAT CAACGGACCATTGATTGAATACTCTAAGAACCGCAGAAAAGCCGATAGTGCCACGACC TCGTTTCTTTCTCCACACTTGCATTTCGGGGAAGTGAGTGTGAGAAAAGTTTTCCATCTTG TCAGGATCAAACAGGTGGCATGGGCAAACGAAGGGAACCAGGCTGGTGAAGAAAG CGTGAATCTTTTCCTCAAATCTATTGGTCTCAGGGAATACTCAAGATACATAAGCTTTA ACCATCCATACTCTCATGAAAGACCGCTTCTTGGCCATCTAAAGTTTTTCCCTTGGG CTGTGGACGAGAACTATTTCAAGGCGTGGAGGCAAGGCCGGACTGGATACCCTTTGG TCGATGCCGGGATGAGAGAGTTATGGGCTACTGGTTGGTTACACGATCGCATAAGAG TAGTTGTTACAAGCTTCTTTGTTAAAGTGCTTCAATTGCCATGGAGATGGGGGATGAAG TATTTCTGGGACACACTTCTTGATGCAGATTTAGAAAGCGATGCTCTTGGTTGGCAATACA TTACTGGTACTCTCCCTGATAGCCGAGAGTTTGATCGTATTGATAACCCACAGTTTGAAGG ATACAAGTTTGATCCAAATGGGGAATACGTACGGAGATGGCTTCCTGAACTTTCAAGACTC CCTACAGAGTGGATACATCACCCATGGAACGCACCTGAATCTGTGCTTCAAGCTGCTGG TATCGAGCTTGGTTCAAACTATCCTCGACCAATTGTAGGATTAGATGAAGCAAAAGCA AGGCTTCATGAAGCACTTTCACAGATGTGGCAACTAGAAGCTGCTTCAAGAGCTGC AATAGAGAACGGATCCGAAGAAGGACTTGGAGATTCTACTGAGTTTGAGGAAGC TCCTATCGAGTTCCCTAGGGATATCACAATGGAAGAGACTGAACCAACCAGACTCAACCC TATTAGGAGATATGAAGATCAGATGGTTCCAAGCATTACTACTTCTTTGATCAG ACCTGAAGAAGACGAAGAATCGTCTCTGAATGTGAGAAACTCAGTAGGAGATAGCA GAGCAGAGGTTCCTATGAACATGGTCAACGCCAACCAAGCTCAGCAGCAGCCGAGAGCA GAACCGGTTTCAAACCAAATCACAGCTATGATTCCAGAATTCAATATCAGGATTGTTG CAGAGAACACTGAAGACTCGACAGCTGAATCTTCAAGCAGCGGAAGGAGAGAGAGGGA CGGAGGCATAGTCCCCGAGTGGTCAGGGTACTCAGAACAGTTTGCTAGTGAAGAAA ATGGGATTGGAGGAGGAAGTACAACATCTAGCTACTTGCAGAATCACCATGAAATACTC AACTGGAGACGGCTTTCACAAACTGGG

TRINITY_DN67410_c0_g1 ATGATAAACATATGTTCTGAACCCCTAGTAGAGGCTACAGTTCGGACCATGTCTAAGTGGTT CATAAGCTCAGGAAAGCTTGAAAATGATCAGAGGTCTTTGCTTGTGGAGGCTTGTAAACT GGCTCTAATCACTCGTTGGGAAGGAAAACACCATATTTATTTCTGGAAGTACCGGATCT CAGAAGCACTTTTTAGTCTTGTTGTGGAGAATTTTCGTAGTCAATCCTTGGAGAATTATGT GTCGCTGGAAAAAGAAATTTCAGTTGCTGAGAAGGTACTGAATGCGAATTTTCTTCCTAGT CTGAGGTCATATGTTTGGGATATAATTGGATTCTTGGCAGCTCACTGTGAGGAAGAGTTC GATTCCATTTTGCGTGGAGATGAACTCCGCCTCAACTTTCTTGTCACATGCGCATGCTTG ACCTTTTCAAGGTCGGTTCAGAAAGGCTATCAAATATGCCAAAACGATATTATCAGCGCCT CCCAAAGTGAGTCGGCGTCAAGAGCAGTGCTAATGATGATATATTCTCCCTCCAAATATA TATCATCAAGGGCCAGAGTTACCCTCTCGTTTATCCTAGGAGAGGATGGTGAACAGAAT TTGAATTCCCTTGTAAATTTCCTAAGTTATATTCCATCTTCTGGAGGTTATGTACTGCCAAA TATACTGCAAACTACCGTCTGCTTAGTGGGCTTGGCATGCTATTCATCGATCCCTCAGT ATGCGAGTTTCATTTTAAGAAACCAGGGTTTGGAGATACTGATATCCTTCTGTAGTTGGT ATCAAAGGAATCGTGACAATATAGGACCATCGAGTTTTGCTCCTTATCCCCAGAGCATTGC TGAGAAGAGGATATGTTGCTGGGAATGTACGGAAGACTGGGACAACAAAGATGCTATT CTTCTCTATGCTCTCTTGGCTTTGGCCGAGTTGGTTACTCATTCTTTCTCTGAACAGAATCA CGCCGAAGAATTTTCAATGAAGAGAGATAGCGTGAAAGATCTGTTATGCACCACACTTCA GGAGATCCGAGATGGAAGTTATGGCTCTGGTCCAAAGTGGTATGCTGCACACATCCTAA CCTATCTTGGATATTATGGCTTCCAACATAAGTTGGGGAAAAGGCTCATGGGAGCTTACG AAAATGAGGAATGTAGCGATCTGCGATTCCTTTTTGCTAGTGGGAATAGTGCGAGCCTA CACAAAGTTATTCTCGCTGTCAGGTGCCCAACGTTACTGCCTCCCAAGGAGGGAGCTC ACAGTGGCTCAATTGAATCAAATGAGAGATCGCAGAGAACCATTCAAGAAATCCGCATG TCTGCGAATGTGGATACTTGGGCGCTTGTGAAGTTGTTGGAATTTGCTTACTCTGGGTAT GTGGAAGTAGAAAACACGGCACGGAAAAAGTTGAAAACTTTGGCGAGACATTGTAAGT CAAAGGTTCTGCTGCAGATGCTGTGCAGAAAAAGACCCAGATGGGGATCTTCAATACC CAGAATTGATCTACCACTTGCCCTCACTCCTAAACTCATTCATTTCTCGGATGTTATATTA GTACCTAAAGAAACAGACATAGCTGGCTTCAACTGCTGTTCGTGTTCCTTTACATCTCC TCATGCTCACTCTCACCGAGTTATACTGTCGTCAGGCTGTGAATACCTACGTGCCTTGT 
Supplemental Table 2. Continued.

\begin{tabular}{ll}
\hline Gene ID & Coding sequence
\end{tabular}

TCCGCTCCGGGATGCAAGAGAGCCATTTGGATAGACTAAACGTTCCTGTGAGTTGGCT GGGGTTAACCAAGCTTGTGAACTGGTTCTACGGTGATGAATTGCCAACCCCTCCATCGG GTTGCAAATGGAATAACATGGACACCGATGCAAAACTACATGAGCTTCAAGCTTACGT GGAGATATACTCGCTCACCGAGTGGTGGATAATGGAAGATCTTCAGAACGACTGTGCT AAAGTGATTCTCTCTTGCTTGGAATCCGCTAGAGAGTTATCGATCAAAACAATCGAATTA GCTGCAAGTTTCTCGATGTGGAAACTGGTGGAAGCAGCTGCTGAGCATGCAGCTCCTAT ATACCATCAGCTTCGTGACTCAGGGGAGCTCGATGAGCTAGATGATGAGCTGGTTAACT TGATCCGAACCGCTGCTGTTCAGTTTTCTCAACAGGGTGGT

TRINITY_DN69128_c1_g2

ATGGCGAAAACACGACCTGGTGTCGCTTCTCCGAAGAGCAAGCTGGGCAAGAGAGATATCG ATTCGTTTACTATCAGAGGCACCAACAAAGTTGTTCGAGCGGGAGATTGTGTGTTAAT GCGTCCAGTGGATGCTGGTAAGCCTTCTTATGTGGCACGTGTTGAGAAAATTGAAGCGG ATGCAAGGAACAATGTGAAGGTGCATTGTCGATGGTATTACCGTCCTGAGGAATCAGAT GGAGGAAGGAGACAGTTCCATGGAGCCAAAGAGCTTTTCATCTCTGATCACTTCGATGTT CAGAGCGCACACTGCATTGAAGGAAAATGCATCGTTCACACCTTTAAAAACTACACGAG GCTTGAAAACGTTGGAGCCGAAGACTATTATTGTAGGTTCGAGTATAAAGCTGCTAC GGGCGCATTTACCCCTGATCGAGTTGCTGTG

TRINITY_DN62817_c0_g1 ATGGCGAAAACTCGACCTGGTGTCGCCTCGAAAATCAAGCCTGGGAGGAAGGAGCTGG ACTCTTATACCATCAAAGGCACAAACAAAGTTGTGAGAGCTGGAGATTGCGTGTTGAT GCGCCCATCGGATGCTGGTAAGCCACCATACGTGGCCCGTGTTGAGAAGATCGAAGCG GATGCAAGGAACAATGTGAAGGTGCACTGTCGATGGTATTACCGCCCTGAGGAGTCTCT TGGTGGTAGGAGACAGTTCCATGGAGCTAAAGAACTTTTCTTGTCTGACCATTTCGATG TTCAAAGCGCACACACCATTGAGGGAAAATGCATTGTTCACACCTTCAAAAACTACACAA GGCTTGAAAACGTTGGTGCGGAGGATTATTATTGTAGATTCGAGTACAAGGCTGCTACTG GCGCGTTTACCCCTGATCGTGTTGCTGTGTACTGCAAATGTGAAATGCCTTACAATCCAG ACGATCTCATGGTGCAATGCGAAGGCTGCAAAGACTGGTACCATCCTGCGTGTGTTGGC ATGACGATTGAAGAAGCAAAGAAGCTTGATCACTTTGTATGTGCTGAATGCAGCTCTGA TGACGATGTCAAGAAATCGCAGAACGGGTTTACTGCATCTCCGGCTGATGATGTCAAGGT GGAAACGAAGCGCAGGAAAAGA

TRINITY_DN50493_c0_g1

ATGCACTGGGTTGATGGTGCTTTGCTGAATGGGGAAAGGCCAGACAGATACAGTTTGTGGTT CTTGGTCCTGTCTGGATCTGATCTACGCTTAAAGTTGCTCCAGTTCTTGCTGCAGAACATT CTGTCTGTTAAGGTGGAAACGAAGCGCAGGAAAAGA

TRINITY_DN58990_c1_g1

ATGAAGAGAGGGAAAGATGAGGAGAAGATACTGGAACCGATGTTTCCTAGACTTCATGTG AATGATGCAGACAAAGGAGGGCCAAGAGCTCCTCCAAGAAACAAGATGGCTCTCTATG AGCAACTTAGCATCCCTTCTCAGAGGTTTAGTGATCATGGTACCTTGCCTCGTAACACA ACCACTTTGGTTCCTTCCGGACCATCTAACCAGCCTTGTGGCGTGGAAAGAAACTTACCTG CGCAACATCTTGATTCTTCAGCTGCGGGCCAAGCAACTGATAAGTTCGTCTCCCAAATG TCCTTCATGGAAAATGTGAGAACCTTGGCACAGAATGATCAGAGGAAAATAGTAAGA GAGGAAGATGATTTCGCAGTTCCCGTATTTATTAACTCAAGAAAGTTTCAGTGTCATGGCA GAAGCAAGAGTGGCATTGTGAAGGAGAAACACTCCACAATCGTAAGAGACCAAGTCAAG GGGAGTGCAAAGCGAGGTGGCTTTGTGAACTCTTCAGAAATATCAGCTACAGAGGAGATG GATCTTGAAGTATCAGCATCGAGTTGTGACAGAGTAAATGATTGCAGTGCTTCCGTGAG ACAAGAGTCTAGAAATTGGTTAGACCGAGATGGTGGTGAAACACAAGTGATGGACAG TAATAACGGAGTCGAATCTCACTTGGCATCGGAAAGTCATACAGAAGAAGGTCGTGGCA ATCCTAATGACGTTGAGAATGGTCGTGAACACTGCAGAAGTGGAGGATACGCCTCTATG CAGCAGATAAACGAAGAGGCAAGTGATGATGTTTCTGATAATTCGATGGTGGATTCTA TATCCAGTGTAGATGTCTCCCCCGATGACGTTGTGGGAGTATTAGGTCAAAAACGTTTCTG GAGAGCAAGGAAAGCTATTGCCAATCAACAAAGAGTATTTGCTGTTCAACTATTTGAGTT GCACAGACTAGTCAAGGTTCAAAGACTTATTGCTGCATCACCGGATGTCTTGCTCGATGA CATCAGTTATCTTGGAAAAGTTACTGCTAAAAGCTATCCAGTAAAGAAACTCATTCCATCA GAATTCATTGTAAAGCCTCCTCTACCACAGGTTACCAAACACAGAAGCGTCGACTCAGA CAAGACTGACCAACATAAACTGGAATGTTCAGCTGAGAACGTGGTCGGAAAGTTGTCAA ACCAAGCTCATCACCATCATCAACAACCTTCCAACTACATGCCTTTTCCGACCAACC AACCCACCGCTTCACCAGGTGTAAACGGATGTTGCTATCCTCCTCATCCTCCTACCTCCG GAGGAAACCAGCAATGGTTGATCCCTGTAATGTCTCCTTCCGAAGGGCTGATATACAAGC CGCACCCTGGTCCGGGACACACAGGGCCGGTCTGTGGAGGGTATTATGGTCATTTCATG CCTGCACCGATGGTTATGGGTAACTTCATGGGTGGTGGTGGTCCGCCTCCTCCGTTC CATCCTGGTAATGGCTACTTCCCTCCATATGGTATAATGCCTCCTACCATGATGAACCCTT

Continued next page 
Supplemental Table 2. Continued.

\begin{tabular}{ll}
\hline Gene ID & Coding sequence
\end{tabular}

ACTATCCTGGCCAACAACAGCAACAACAACAACATCAACCAAATGAGCAAATGAATCAG TTTCAGAACGCATCTGCTTTGAACACTCAACAACAGAGCTCTGTGAATGAAGCGGCTGC GCAACAGCAGCCGACGAAGTCTTATCCTCGGGCTAGAAAGAGCAGGCGAGGGTCTACA GCAAGCAGTCCAAGTGGTCCAGAGGGAATCTCTGGTAAGAACTCGTTTCGGCCATTCTT AGCGGTTGATGATGACGACAACAACAACAACATCAACAACGCACCTGAGCAAATGATGAC GACAACCACAACGACGACAAGAACTACTGTTACTCAGACAACAAGAGATGGAGCAGGA GTGACGAGAGTGATCAAGGTGGTTCCACACAACCCCAAGCTGGCGAGTGAGAACGCTG CGAGGATTTTCCGGTCAATACAAGAAGAGCGTAAACAGTACGACTCCTTCTCTAACTA CCCT

TRINITY_DN69071_c0_g2 ATGGTGCTGCTATCTCTGCTTCTTCATCTTCAGAGGCAAAAAAATGAACTTGAGGAGGGTC ATTCTGGAGTGCAGAATAAAGAGAGCACTAATCTTTTAGAGGAGTTGAGTTTGTTCATGG AGAAGCTGAAAGATGTATATTACGACGATGATGATGGTCTGCTTAATGACTTCCAAGT TGATTCTGGAACCTTGGCATGTGTGGCGTGTGGCGTTCTTGGCTTCCCCTTTATGTCTGTG GTACAGCCTTCTGAAAATGCATTAAAAGATCTCTCAGAGAGACAAGGCGAGATAGAT GCTCAGGATATTACGGCACTGTCATTAGAAAAGTGTGGCGGTGAATGGAACACGTCATC CAGATATATAAGACCTCGCATTTTCTGCCTCGAACACACTATTGAACTTCAGAGACTG CTGCAGTCAAGGGGTGGACTGAAGTTCCTTGTAATCTGCCATAAAGATTTTCAAAAATTTA AGGCACATGCGGCTATAGTGGCAGAGGAAGTCAAAGTCCCTTTCAGCTATGATGATGT CCTGTTAGAGAGTGCATCTAAAGAAGAGTTGAGTCTAATTGATCTTGCAATCGAAGAT GAAGAAAACAACGAACACGGCGTAGACTGGACCTCAAAACTTGGTATCAATTTACGGTA CTGTGTTAAAGTGAGGAAAAATTCCCCTACTAATAAAATTCAGCATGCACTGTCACTAGGT GGCTTGTTCTCCGACACAAGCCACATGCTAGATATGTCAACTATCAAATGGCTGCAGAGA AAATCACGCTCAAAGGCTAACCCCAGCTCTACCTCAAGTTTCACACCTCGTGAACATCT TGAAGTAAAAGTAGACAGAAATTTAGGGGAGGAAGGAAGAAAAGATCATCCAGTA CTCGAGAAAGAAAATGTTGAATCCCAAGCCGGAAGAAAGGAAGAAAAGATCATCCAG

TRINITY_DN51551_c0_g1 ATGGGTAATGTTGAGATTCCCAATTGGCTAAAAGCCTTGCCATTGGCGCCTGTCTTTAGACC TACAGACACCGAGTTCGCAGATCCTATTGCGTATATATCGAAAATCGAGAAAGAAGCCA GTGCCTTTGGGATCTGCAAGATCATTCCTCCTTTACCAAAGCCGTCGAAGAAGTATGTTT TCTACAACTTGAACAAGTCTCTTTTGAGGTGTCCTGAATTGGCTTCGGATGTAGACAT TTCGAAAGTGTGTCAGGAGGATAGAGCTGTATTCACCACTAGGCAGCAAGAGTTAGGCCA GGCTAGACAAAAAACCAAAGGACGAGAGAGCAGTAATTCTCAAAGGAGTGGTGTTAA GCAGGTGTGGCAAAGTGGTGGCGTATATACATTGGAGCAGTTTGAGTCAAAGTCGA AAACTTTCTACAAAAGCCAGTTAGGAACGATTAAAGAAGTGTCACCAGTTGTGGTTG AGGCATTGTTCTGGAAAGCAGCTTCAGAGAGGCCTATATACATAGAGTATGCAAATG ATGTGCCTGGCTCCGCTTTTGGTGAGCCAGAGGGTCATTTGAGGCATTTCCGGCAGA GAAAGAGGAGGGGGAGAGGATCTTATCAGAGGAAGGCAGAGATCAATGAGGACAG CAGCGTCGAAAAAAGGACGGAAGGAGACAATTATCAAGAACCATCCTGTAAGAATGG TGAGAAAACATTGCCTGAGGTACCAAAGGCATCTCTTGCTTCTCCAAGTTCATTA TCTCAAGATCCGTCCAAGAGGAAGAACATG

TRINITY_DN69458_c0_g3 ATGTCTCCAACTCTAAGTAGTCATGTTGTGGACCTTGTGGTAAGTCCGACCATACATGCT TCTACAGTCGATAATCATGATGGCCCAATAAAGTTGACCGGTGAGTACATCTGCAGCTA TGTGTCTGTACGTAATGTTGATGAAGCGGTTGAAAGGAGCGACCAAGAGTTCGAAGA ACCTAGGTCTACTGTCGCTAACATTGAGGAGGAACAGCAATCGCAGGTGGTGCAACCAAC CGAAAGAGAAGCTGTATTTGGTGATAACACGCAGGTGGAGGGAGAGGAAGCTATGTGT ACCATAGAAAACTTGTGCTCTGAAGATATTATGCATACTGAGCAACAGGAAGAGCAAACA CATTCTTCAGCTCAATTGGATTTAAAAGTTCCTGAAACTAACATTGCCAGTGAGAACAT AGTTGTGGACATAATTCATGATGATGAACCTCTGGCAAGCAACTGTGATCAAGCGTCTTCA AATGGACTGCAAGCTGCAGATAATGAGATTAGCATGGATAGCGAAGTTGCAAGCTCAGG AAACACCGAAGTTATCGAGGCGCCCAATTCTAATATGGGGAGAGCAAAGAAGAAGCG GAAAATGGAATTAGAGTCTGAGACAAATGATAATCCAGAGAGTAGCATTGGTTTCA TAAGGAGTCCTTGTGAAGGGTTGAGGTCAAGGGGTAGGAGGAAAGTGACATGTGAAACA TCATTCAATCTCACTGAAGCGAGCGACGAAGAGAAGAAACCCACTGCGAAAAGGCTCA AGAAAACTCCAAATACTCGC

TRINITY_DN95956_c0_g1 ATGCCCTGTCTTCCGTCTGTGGCGGCTATAATCCGACACAAACCTAAACGACAGTCCACA CTCGTCGACCTTGCATTTGTATGGTCGTTCTCCAGTGTGCAGACGCAAATGCTCGG TCCTCGCCCACTGCCATTTGAAGTTCATGGAACATCCTTTCCAAGGGCACGCGAA AGGTCTATCATCGTTATGAACACGTTGATGAAGCACCAGATATTTGTGAGCCCTGAAT TTCTTTCCACACCCTTCCTGTGTGCAGCGGTTTCTCTTGTGAGCTTGCAAGTCCGCTCTA CTCTTGAAAGTCATCTTGCATCCCTC 
Supplemental Table 2. Continued.

\begin{tabular}{ll}
\hline Gene ID & Coding sequence
\end{tabular}

TRINITY_DN69534_c0_g2

ATGCGTAATGAGAAAAGAAATCCAAAATTAGAAGCGACACATCTGGAGAAGGCCA AGGAACTTTATACTAAAGTCCTGACTCAGCATAATTCCAACATGTATGCTGCCAACG GTTCTGGCATTATATTAGCAGAGAAAGGACAATTTGATATTGCCAAGGATCTTTTCA CTCAGGTTCAAGAAGCTGCAAGTGGAAGTGTGTTTCTTCAGATGCCTGATGTAT GGGTGAATCTGGCTCATGTGTACTTTGCTCAAGGGAATTTTGCCTTAGCCGTGAAA ATGTATCAAAACTGCTTGCGGAAGTTCTTTTACAACACAGACTCTCAAATTCTTCT TTACTTGGCCCGTACCCATTATGAGGCTGAGCATTGGCAAGAGTGCAAAAAGA CGCTGCTAAGGGCCATTCACTTGACTCCTTCGAATTACACATTTCGATTTGATTTGG GTGCTGTAATGCAGAAATCATCATCTTCCACACTACAAAAGAAAAAGAGAACA GCTGATGAGGTGCGCTCAACAGTCACGGAAGCAGAGAATGCTGTTCGCGTATTCA CTCAGTTGTCTGCTGCGTCAGACCTCCATGTTCATGGGTTTGATAGTAAGAAAATAC AAACCCATGTTCAGTATTGCACGCACTTACTGGAAGGAGCGAAAGTTCACCGTGAA GTAGCTGAGCGTGAGGAGCTGCAGAATCGGCAGAGACTAGAAGTTGCTCGTCAGGCTG CTTTGGCAGAAGAAGCACGCCGTAAAGCTGAAGAACATAGGAAATATCAGTTGGAAAA AAGAAAACAGGAAGACGAGCTGAGACGCCTCAAGCAAGAAGAAGAAAAAATTCAACG TATAAAGGAACAATGGAAGAGCAACCCACATGGATCTCATAAGCGTAAGGATAGAG TTGAAGATGATGATGGGGAAGGTAGGCCCAGTGAGCGGAAAAGAAAGAAAGGCGGGA AGAGAAGAAAGAAGGACAAAGGCTCAAGGGCTCGACACTATGAGGACGATGATGAAG AAGCCGCTACTATGGATGATCATAATGAAGTGGAAGACGAAGACGCCAACACTA ATTATAATAGGGAAGAGGAGAATCAAGAAGCTGAGGAGCCTGTGGATGATGATGCTCAT GACCTTCTTGCCGCTGCAGGGCTCGAAGATCCTGATGCTGATGATGAGGTACCTGCTTC AGTTGCGAGGCGAAGAACGGCGCTTTCGTCATCAGATGAAGAAGGTGAATTGATGGAG AGTCAGCCAAATTCAAGCCCCCAAAAAGAGAATTCTCCGGGGAGGCAAGAAGAGAGC AATGTGGAAGAAGAAGAGACTAACCTGGACAACGAGGAGGCCAAT

TRINITY_DN67027_c0_g1 ATGACCTTTGCTGAAGAAGACGAAGTAATTCCGACAATAAACATTCTTGATACTTCTGTTG ATAAGGAGGATGCCTCGAAGGATGATGTGGCTGCTAAAAAAGAAGAAGATGGCTCTG ATCAGACCGCGGAAATAAACAACAACGGCAAGAGGAAGCTGCCAGAAAAAGAAAC TGAAAAGAAGGAAGCAAACAAGCCTCCGGAATCGTGGTTTGAGTTAAAAGTAAACCCAC ATATATATGTGACTGGGTTACCCAAGGATGTCACCATCGAGGAAGTAGTTGAAGTTT TTTCTAAATGCGGCATAATTAAGGAGGATGACACTGGTAAGCCTCGCATAAAGCTTTACA GTGACAAGGCAACAGGAGAGTTAAAAGGCGATGCTCTTATCACGTATATGAAGGAGCC GTCAGTTGATCTTGCAATTCAAATCTTAGACGGGGCTCCTTTACGTCCCGCTGACAAGCT CCTCATGTCAGTTTCTCGAGCTAAATTCGAGCAAAAAGGGGAGAGATTTATAACGAAGCA AACCGACAACAAGAAGAAAAAAAAGCTTAAAAAGGTCGAGCAAAAGTTGCTTGGAT GGGGTAAGTTTTTTTTTTTTTCTTTGAAT

TRINITY_DN67027_c0_g2

ATGTCCAGCTCCGAGAATCTACAGGTTCCACAATCATCTTCAGACTCTACTGATGTTGGTT GGTACATTCTTGGTGAAAACCAACAAAACCTCGGTCCATATACCTTCTTGGAGCTCTGTG ACCATTTCAGGAATGGTTACCTACAGGAAACTACCCTAGTCTGGGCTGAAGGAAGA AGCGAATGGCAGCCACTTTCTACCATCACTGAGTTGATGTCAAGGATTTCTGGAGCTGA GGTTGACTATTCAGCTGGAGGTGCTATAAATGGATATAATGCTGGAACCAACCAAGAGAA GCAAGATAATACTGCCTCT

TRINITY_DN57304_c0_g1 ATGGGATCCTCAATAAAGATCAACGCAATATCCATAGATCTCGCAGGCGCTGCCAACGA GATTGATATGGTAACATGTGATCATTTCTCCATACGTGGATTCGTAGCTGAAACGCG CGAGAGAGATCCTAAGAAATGTTGGCCGTTTTCGGAGGAGAGTGTTAGTTTGGGAGA CCAACAAAGCCATTCCCTTCCTTCTTTGTCTGTTCCAAAGTTTAGATGGTGGCGTTGCA TGAGCTGCATCAGAGATATCGATGCTGATGGTACAAACGATTGTGGACTGCCTTCAAAC TCGAGAAGTATAATTAGCGGGAAAAAGCTCGATGGAAGCTCTTCGGTCATACCTAGCC AAAGCAAACCGAATACTCTAACTGTCGTTGATCAGGAGAAAGAAAGGAATATTAATAT CGCAGGTAGTGCTATTGTGGAGAAGGAGGATGTAAACTGCGAAAGTTCTCGGAAGAAT GATCAGAGAGCTACTACGTCTCTCAAGAAAGTTCATCCTCCATCTATGAATGTTTCT ACTGTTAGGAGCAAGGGCAGAAAGCTGGCGAGTCCAGAGCAAGTAGGAAACAAGAGA CCTAAAGTCAACAAATCCTCCACGGATATTAGCAGCGTGAAAGAGACACAAAACGTGGA TCAGCTGGCTGTGACAACTGTTGCCTCATCTGAGATCGCTCGTGTGGTTGAGGATAGGCC GCCTAATGAGTCATCAGAGAGTAGGAAGCTTGCCACAAGTGGGTTGCAGCGTAGGA AAACTCGTCGAATGAGCGAGCTGGTTTACAATGATACCCAGAAAAAAGCCGAAAGGAAGC CGACTACCAGCAAAAAAGAGTCTTGTTCGAAAAAGGAACCGGTTAAAGGTACAAA AAGAAAGGTATCACCTGAAAAAATTTATGCCAGCAGGAAAGCCGGCGCAACTTCTG 
Supplemental Table 2. Continued.

\begin{tabular}{ll}
\hline Gene ID & Coding sequence
\end{tabular}

AAAATGCCTCCAAAAGCCGTGGCTCTGATCAAGGTGATAGTGAATCAACTGATAGTGGGT TTGACAGAGATCCTATTAACGGTAAGCAAAAGAACAGAAGATTCCAGATTGTTGAC GAGTTTGTAGTACCATCATGTCCTTCCCAAGAGGGCGTCCGTGAGAATGATGCAGAT CCCGGTAAGAGTGCTTTATCAACTCCTGTGCACTCTTTAGTCAAGGAAAAAGATTCTGT TCCTTCTCCTCTGAGAGCTCAGAGAACAGATCAGAAGCTCAGTTTAGCTAGGAA GAAGAAAAATAAGTCAGAAATAGATAGCGTGAAGAGTAGCCTAATCAGTTTTAGT TCCGGAGTGGATGAAGGAAGTCAGGTCAAACCGCGAACCGGTCCTTCCATAAACGC AAGTTCCCAACCTACTCGGGAATTCTTGAATGAGAAAAGGGTGGGCAGTGTACTTG ACGATCGTCTTGCTTCACAAGGATATGTCAGAAAACCTATCCCTCAGGTTAATGACAG GCCAGTCCTTTCTTTGCATATGCCAGACAATGAGCATATGAGGTCAACAGATGCAGAA GCAAACTGTGTTCGAGAGTTCGGTTCCTCTTCTAATTCCAACACAGGTGGATGGCTGA GTGCTCTAGTGGATGCTGTTGACTTCAGCAGCAACAGCAACTCTAATCTAAGAATC ACCTCGTCTTCTACACAAGTTGCAGACCCATCTTCTGTGACGCAAAAGGATGCTTCTGGT GCAGACAGAAAGGGGAAGAGAGTAATGGTCCAAAATCATCATGGAGCACTCGGAA GCCAAAGCCATGACAGAAAGGAGAATAGGCCAGAAGAGCAAAACGGTGATATTCTCAAG GCTATAGCCGAACTCCTGGCCAAAAAAACTAGACGAAAAATGTCTAACCAACAAAGA AGAGGACGTTGGCAACAAGCAGCCACCACAAGAACCAGCACACAAATCCAAGAATGCTC TACTGAT

TRINITY_DN66245_c2_g3 ATGCCAGGCATTCCTCTCGTGACTCGTGAAACTTCTTCTTGTTCAAGAAGCACAGATCAGA TGTGCCATGAAGACTCCCGTGTGCATATGTCTGAAGAGGAGGAGATTGCTGCTGAAGAGA GCTTGGCTGCGTATTGCAAGCCTGTAGAACTCTACAACATTCTTCAGCGGCGTGCTATTA GGAATCCCTTGTTTCTTCAAAGATCTTTGCATTATCAGATTGAGGCAAAACATAAAAGG AGAATACAAATGACTGTGTTCCTTTCGGGGACTATAGATGCTGGGGTACAAACTCAAAA GCTGTTCCCTCTCTATATCCTGTTGGCAAGACTCGTTTCTCTTAAGCCTGTTGCCGAGT ATTCTGCAGTATATAGGTTCAGTCGAGCATGTATTCTTACTGGTGTCCTGGGGGTTGATG GAGTTAGTCAAGCACAAGCCAACTTTCTTCTCCCTGATATGAATAAACTCGCGTT GGAGGCAAAATCAGGATCACTCGCTATCTTATTCATTAGCTTTGCTGGTGCGCAAAATT CACAATTTGGCATTGATTCAGGCAAGATCCATGCAGGAAATATAGGAGGACATTGCC TATGGAGCAAAATACCTCTTCAATCACTCTATTCTTCGTGGCAGAAATCTCCAAACATGG ACTTGGGACAGAGAATAGACTCAGTCTCTCTTGTTGAAATGCAGCCTTGCTTCATAAAGT TAAAGTCTATGAGTGAGGAAAAGTGTGTCTCGATTCAGGTTCCCAGCAATCCCCTCACCTC GAGCTCACCGCAGCAAGTGCAAGTCACCATATCTGCAGAAGAGGTTGGGGCAACGGA AAAATCTCCTTATAGTTCATTTTCATATGATGACATCTCTTCCTCTTCACTGTTGCAAA TTATCAGGTTGAGAACACGGAATGTAGTTTTCAATTACAGATACCATAACAACAAAC TGCAGAGGACTGAAGTAACTGAAGACTTTTCTTGTCCATTTTGCTTAGTAAAATGTGCCA GTTTGAAGGGCCTCAAATATCATTTGCCATCAACCCATGATCTCTTCAATTTCGAGTT TTGGGTAAGTGAAGAATATCAGGCTGTAAATGTGTCTCTCAAGAGTGAGACAATGATC GCCGAGATTAATGAGGACGGCGTTGACCCGAAGCAGCAAACATTCTTTTTCTCTTCA AAGAAAGTCAGACGTAGGAGGAAAAAGAGTCAAGTACGGAGCTCAACGCAGGGCA ATCATCTTGGACTAGGCTGCGAGGTGCTGGATAAGACTGATGATGCTCATTCTGCGAGAA GTGAGAAGAGCCGAATACCAGCTGGAAAGCAGTGCGAAAGAATTGGGGGTGCTGA GTCGTCTGGTCAAAGAGTTCCTCCTGGCACGAGTCCTGCAGACGTGCAATCAGGTGC TGATCCAGATAATGTGCAGTCGATAGCTGGAAGTACGATGTTGCAATTCGCAAAAAC GAGGAAGCTATCTATAGAACGGTCGGACTTGAGGAACCGTAGCCTCCTTCAGAAGAGACA GTTCTTCCACTCTCATCGAGCTCAGCCCATGGCTCTAGAGCAAGTACTTTCTGACC GAGATAGTGAAGATGAAGTTGATGATGATGTGGCAGATTTTGAAGATAGACGGATGCTCG ACGATTTCGTTGATGTGACCAAGGATGAGAAGCAGATGATGCACATGTGGAACTCG TTTGTGAGGAAGCAGCGAGTATTAGCAGATGGTCACATTCCATGGGCATGTGAGGCGTT CTCAAGATTACATGGACCCGTCATGGTTCGAACACCCCATTTGATCTGGTGCTGGAG AATGTTTATGGTGAAACTGTGGAACCATGGCCTTCTTGACGCCCGGACCATGAACAGC TGTAATACCTTTCTCGAACAGCTCCAAAGT

TRINITY_DN70231_c1_g3 ATGCTTGATTCCTTGTCTTTAGGTAGAGAAGAGACGAGCCTCGAAGCCTATAGGAAGCTTTT GCAAAGTGCAGAGAGGCGGAACCCGAAGTTGGAAGCTTTGGGTTTTGAGATTTTGT TTAATGAGAAGCGCTTGTCACAGCTTCGCCAGTCTCGCCCAAAGCCTGTGGAGAAGCC TCTCAAGAAGGTGCCTCTTGAACCTTTTATACCTCTCACAAAGGAGGAGGAGGCTGAAGTC TATCGAGCCTTTTCTGGCAAAAACAGAAGGAAAGTCTTGGCTACTCATGCAAATTCG AACATTGATATTACTGGAGAAGTTTTACAATGCCTTACACCATCTTCATGGCTCAACGACG AGGTTATCAATGTCTACCTTGAACTACTCAAAGAGAGAGAAACTAGAGAGCCCAAAAAGT ATTTGAAGTGTCATTTCTTCAATACCTTT

Continued next page

J. Amer. Soc. Hort. SCI. 143(1):1-38. 2018. 
Supplemental Table 2. Continued.

\begin{tabular}{ll}
\hline Gene ID & Coding sequence
\end{tabular}

TRINITY_DN64502_c0_g2

ATGTTGTCATCAGCAAAGCATCAGAGAAACCATAGAGTCTCTGCAACAAACAAGAACAAGA TTCTCAACAAAGTTTCTTCCATTTCATCCTCATCATCACCACCATCATCATCACCATCATCA TCCTCATCATCCTCATCCTCATCCTCATCCTCATCATCTCCTTTACACTCACAGGACTCCCA AGGCCAGAAGAGATCTCTAATCACCATGGAAGAAGTATGGAAAGACATCAACCTTGCTTC CATCCACCACCTTAACCGCCACAGCCAACATCCACAACACAACCATGAGCCAAGGTTCAG GAGCCACAACCACCAGAACCAAAACCCTAACTCCATCTTCCAAGATTTTCTTAACAGACCT TTGAACCAGGAACCAACAATACCCACAAGCCTCACCATGGGTTCTTCCTCTAATGGCGATA CCACCACTGTCACTGCTCTCTTCAGCAGCTCTCCTTTGGCACCTCCTGCAACTGTTCTGA GCTTGAATTCCGGTGCTGGCTTCGAGTTTCTCGATAACCAAGATCCTCTTGTCACCCCTAA CTCTAATCTGCATAGCCACAATCACCTCCCAAACGTTTCTTCATTCAACACCCCTTTT GAGGCTCTCGTTACATCCACTTGTTTTGGTAAGAAAAGAGGCCAAGATTCCAATGAAGG TTCAGGGAATAGAAGACATAAGCGTATGATCAAGAACAGAGAATCTGCGGCTCGTTC CAGAGCTAGGAAACAGGAATGTGCCTCTCCTCCTCTCCTGACCTTCTACTCGAAAAA GAAATGTGGTTTTGCCATCAAAATGCTTTTGATC

TRINITY_DN69998_c3_g1 ATGGCGAGAGAACATCCGAATGAAACGATCGGAAAACGGAAGAAGCGTGGCAGAGTTG AAGAAGAAGAAGAAGAAGAATACATGGGCGATGGAATCGGAAAGGAAGTAGAAGAAGA TGTAGGAGACGAGAAGCTTCCATTACAAGTTGGGATGTTCTATTACCCAATGACACCA CCTTCGTTTATCGTATCCGATGCTCTGGAGCCAGATTTTCCTTTGATCTACGTTAACA GAGTCTTCGAGGTCTTCACTGGTTATCGAGCCGACCAAGTTCTTGGTCGGAACTGTCG ATTCTTACAGTACAGAGATCCTCGTGCTCAAAGGCGACACCCATTGGTAGATCCTGTG GTTGTGTCTGAGATTAGAAAATGTCTTGAGGAAGGTATCGAGTTTCAAGGAGAGCTTC TCAATTTCAGAAAAGATGGTACTCCTTTGGTTAACAGACTACGGCTTGCTCCAATACG CGACGATGATGGAACCATTACACACGTGATTGGGATACAGGTCTTCTCTGAGACGACT ATAGACCTTGACCGTGTCTCATATCCTGTCTTCAGGCAACAGTTTGACCAGACATCTC AAGACTTGGCTCCAAGCGGGAGCCCGAGGTCCAGAGAGGATCATCAACATGTCTGTGG GATACTCCAGCTGTCTGATGAAGTCTTGGCCCATAACATCTTATTCCGGTTAACTTCA AGGGACGTTGCTTCCATTGGTTCTGCCTGCAGGAGGATGAGACAGCTCACGAAAAACG AAAGCGTGAGGAAGATGGTTTGTCAGAATGCGTGGGGAAAAGAGATAACCGGAACGTT GGAGATCATGACTAAGAAGCTAGGATGGGGTCGGTTAGCTAGAGAGCTCACTACCCTT GAAGCTGTGTGTTGGCGCAAATTCACTGTTGGAGGGATTGTACAACCTTCGCGATGCA ACTTCAGTGCGTGTGCTGTTGGGAACAGGCTTGTGCTATTTGGTGGGGAAGGGGTTAA TATGCAGCCAATGGATGATACTTTTGTTCTCAATCTTGATGCAGAGTTTCCCAAGTGG CAACGTGTGAAAGTGAAGTCATCTCCTCCAGGACGTTGGGGACACACTCTCTCGTGCC TCAACGGGTCGTGGTTAGTAATATTTGGAGGGTGTGGAAGACAAGGATTGCTTAATGA CGTATTTGTACTTGATCTAGACGCTAAGCATCCGACGTGGAAGGAAGTAGCTGGAGGG ACTCCTCCATTGCCTAGATCTTGGCACAGCTCTTGCACCATTGAAGGCTCGAAGCTGG TCGTCTCAGGTGGCTGTACAGACGCTGGGGTGCTTCTCAGCGACACCTTTTTGTTGGA TCTAACGACAGATAAACCGACATGGAGAGAGATCCCGACATCATGGGCTCCTCCTTCT AGACTTGGCCACTCTCTATCCGTCTTTGGTCGGACTAAAATCTTAATGTTTGGTGGAC TTGCAAATAGCGGCCATTTAAAACTAAGGTCTGGAGAGGCATACACTATAGACTTGGA AGACGAGAAACCGAGATGGAGAGAGCTCGAGTGTAGCGCGTTCACAGGCGAGGTTGCA CCGCCTGCAAGACTAGACCACGTGGCTGTGAGCATGCCCTGTGGTCGAGTAATCATCT TTGGTGGGTCTATCGCAGGGCTTCACTCACCTTCGCAGCTGTTCCTTATAGATCCTGC GGAAGAGAAACCATCATGGAGGATCCTGAATGTCCCGGGGAAACCGCCAAAGTTTGCT TGGGGACATAGCACTTGTGTTGTCGGAGGGACTCGAGTATTAGTTCTGGGTGGTCACA CCGGTGAGGAATGGATACTCAATGAGTTACATGAACTCTGCTTGGCTAGCCGCCAGGA CTCGGATATG

TRINITY_DN69832_c1_g1

ATGGGAAGGAGAAAGGTGGAGATCAAACGAATTGAGAACAAAAGCAATCGACAAGTCA CTTTTTGTAAACGACGCAATGGTCTCATGGAGAAAGCTCGCCAACTCTCTATTCTCTG CGACTCCTCCATCGCTATTCTCATAGTTTCATCCACCGGAAAACTCTATACCTCCTCC TCCGGCGATAGCATGGCCAAGATCCTCAAGCGTTATGATATACAACATGCTGATAAAC TTAAGAAAATGGGTCTTGCAGAAAAAACTCGGATTTATCTTTCACACAAGGAGTTGCT AGAAATAGTCCAGTGCAAGCTTGAAGAAGCGAAAAGTGATAACATAAGTGTGGAGTCC CTAACTGCCCTGGAAGAGCAGTTCAAGACTGCTGTGTCTGTGACTAGAGCTAGGAAGA ACGAACTAATGATGGAGATAGTGAAGACCCTTCAAGAAAAGGAGAAGCTGTTGAGAGA AGAGAACCAGGTTTTGGGTAGCCAGCTTACAAAGATGGAGAAGAAAAGGTTTCTG 
Supplemental Table 2. Continued.

\begin{tabular}{ll}
\hline Gene ID & Coding sequence
\end{tabular}

TRINITY_DN67304_c0_g1

ATGATGAATCCACCGGAGAAGAACGAAGATAATGTCCCTTCTGTATCTCACTTTCAAT TTTCCACTCTTACTCCACAATCGAACTCGAATCCTAATCCAGACCCAAGTTTGAATCA ATTCCCGAGCTCGATCTCCAGCTCAAGCCCTAGTTTTGAGCAGATGGTATCATTCTCC GCGCCAAAGAAACGAAGGAGAGGACGGTCTCAGCGCTCAGCGTCCTCGTTTCACATCC TTCCCGTCCCTAACGTCGGCGTCCTTCCCGGTAACAGCAATTTCGTGTCACCTTCAGC TTCTTCTTCCGGTAGACCGAACGTAGAGGGCAATGGATCGAACCAGACTGTAAAAATT TATCCTGGGATAGGTGACGAGATCATCACGATCAACAAGGAAGCTACCACCGAAGCTT TGCTTGCGCTCACAGCTGGGTTCCCTGCTGATTCACTTACAGAGGAAGAGATTGAGTT CGGTGTGGTTCCTGTGGTTGGAGGAATCGAACAAGTGAATTACATATTGATTAGGAAC CACATCATATCGAAATGGAGAGAAAATGTATCTAGTTGGGTCACAAAGGACATGTTTC TCGATTCGATTCCTAAACACTGCACTAGTCTCTTGGATTCGGCTTATCATTACTTGGT CATGCATGGATATATCAATTTCGGTGTAGCACAGGCAATCAAAGACAAGATTCCTGCT CAATCAAGCAAGCCTAGTGTGGTTATAGTTGGTGCTGGGTTATCTGGTTTGGCTGCAG CGAGGCAGCTTCTGAGGTTTGGGTTTAAAGTCACGGTTTTGGAGGGTAGGAAACGACC CGGTGGACGGGTTTATACAAAAAAGATGGAAGGTAATAGAGTAGTCGCTGCAGCGGAT TTAGGAGGAAGTGTTCTGACTGGAACGTTAGGAAACCCTCTGGGGATTATAGCCAAGC AGCTAGGTTGTTCTCTTTTTAAGGTACGAGATAAGTGTCCTCTTTATAGAGTGGATGG TAAACCGGTTGATCCCGATCTGGATATGAAGGTCGAGGCAGCTTTTAACCGGCTTCTT GACAAGGCAAGTAAACTTAGGCAGTCGATGGGTGATGTTTCCATGGATGTCTCCCTTG GGGCAGCGCTCGAGACATTCAGGCAGGTTCATGGGGACGCGGACGATGTGGCCACCGA GGAGATGAGTTTGTTTAATTGGCATCTTGCTAACTTGGAATATGCAAATGCGGGTTTA GTATCAAAGCTCTCTCTTGCATTCTGGGATCAGGATGACCCATACGATATGGGTGGAG ACCACTGCTTTCTGCCTGGAGGAAATGGAAGGCTGGTTCAGGCTTTGGCTGAAAATGT ACCAATCTTATATGAGAAGACCGTTCAAACAATCAGATATGGTGCAGATGGTGTGAAG GTTACTGCTGGAAATCAAGTGTATGAGGGTGATATGGTGCTGTGTACGGTTCCCCTTG GTGTTCTTAAGAACGGGTCCATCAAATTTTTGCCGGAGTTGCCTCAGAGAAAGCTTGA TTGTATAAAGAGACTAGGTTTTGGGTTATTGAACAAAGTTGCAATGCTCTTTCCGTAT GGATTTTGGGGCACAGATCTCGATACCTTTGGACATCTTACTGAGGATCCGAACTATC GAGGTGAATTCTTTCTGTTCTATAGCTATGCACAAGTTGCAGGTGGTCCTCTACTGAT CGCGTTGGTTGCGGGAGAAGCTGCTCATAAGTTTGAGACAATGCCACCCACTGACGCA GTGACTCGTGTTCTTCAGATTCTTAGGGGTATGTATGAACCACAAGGAATAAATGTCC CTGATCCAATTCAAACGGTCTGCACCAGATGGGGCGGGGATCCATTCAGTTTCGGATC TTACTCTAATGTTGCAGTGGGAGCTTCAGGTGATGATTACGACATATTAGCAGAAAGT GTGGGAGATGGAAGGCTTTTCTTTGCCGGAGAAGCCACGACAAGGCGGTACCCTGCAA CCATGCATGGAGCTTTCGTAACTGGTCTGCGAGAAGCTGCTAATATGGCTCATTCTGC AAAGGCCCGAGGCATAAGGAAGAGAATCGATAGGAACCCTTCAAAGAATCCCTATTCT TGTGCTACCCTTCTTGCAGATTTGTTCAGGGACCCTGATCTGGAGTTTGGGAGTTTTT CCATAATCTTTAGTCGAAGGAATCCGGACCCAAAGTCTCCTGCAATTATGAGGGTAAC GCTAAGCGAGCCTCTTAAGAGGAACGAAGACCCAATAGCAGATCAGCATTCAAACAAG ATACTGTTTCAGCAGCTTCAGTCTCATTTCAACCAGCAGCAACAGATTCAGGTGTATA CGTTATTGACTAGACAACAGGCTCTTGACCTGAGAGAAGTCAGAGGTGGTGATGAGAA GAGACTTCACTACCTATGTGAGACACTTGGAGTGAAGCTGGTGGGAAGAAAAGGTCTA GGAATTGGAGCTGATTCCGTCATCGCTTCTATTAAAGCTGAGCGAACCGGTCGCAAGC CAGCGTCATCATCTTCCGGCACAAAATCTGGTTGGGCTGTA

TRINITY_DN40841_c0_g3 ATGGCCGAAGCTGAGGAACAACAAAATTTTGTCGCACACAATGGAGATGAGGTGCATA ATGGTTTACAGTACCAGGTCCATGACGAGACCCTTGCTCACCAACCATACGAGGTTCA AGACCCGATTCTTGAACCTCAGCAGTACGAGGTTCAAGATCCGACCCTTGAACCCCAG CAGTACGGTGGTCAATTAGAGTACCAAGACTTCCAGGTGCAGGACCAGGCTAATGAAG AAGTCCACGATCAGCCGCAAGATGAACTACAATATCAACCTCAAGAGCAGGAGCAGTA TCAGTTGCACGACCAGGCTCATGGTCAGGCGCAGTATCAGGTTGAAGGCGAGGCACAG GATCAGGATGGTGATGAAGTGCAAGATCAAGTTGACGGTGAGGAAGAAATTCCTGAGC ATGTTGAGTCTCTACAGAAATCAGAGCTTGAAAGTGATATAAGTATAACAGGCGGAGG TGAGGAGAAGCGATGGCCGGGGTGGCCCGGAGAAACAGTGTTCCGTATGCTGGTTCCT GCACAGAAAGTGGGTAGCATCATTGGTCGCAAAGGTGATGTCATTAAGAAAATAGTTG AGGAGACAAGGGCTCGGATAAAGATTCTTGATGGTCCTCCAGGCACAACCGAAAGAGC TGTTATGGTTTCTGGAAAAGAAGAGCCCGAATCATCTCTGCCTCCTTCTATGGATGGC CTTCTGAGAGTCCACATGCGGATAGTTGATGGTCTAGATGGTGAAGCTTCTCAGGCCC

Continued next page

J. Amer. Soc. Hort. ScI. 143(1):1-38. 2018. 
Supplemental Table 2. Continued.

$\begin{array}{ll}\text { Gene ID } & \text { Coding sequence }\end{array}$

CTCCGCCTACAAAGGTTTCAACAAGATTACTAGTCCCAGCATCTCAGGCTGGAAGTTT

GATTGGAAAACAAGGAGCAACAGTTAAAGCCATTCAAGAAGCATCTGGCTGTATAGTT AGAGTCCTCGGATCAGAGGATTTGCCTGTTTTTGCTCTTCAAGACGATAGGGTTGTTG AAGTTGTGGGGGAGCCAACAAGTGTTCATAAAGCCTTGGAGCTAATTGCATCACATCT CAGAAAGTTCTTGGTTGACCGTAGCATCATCCCATACTTTGAAAATCAGATGCAAAAG CCAACTCGCCAGATGGATCATATGCCGCCACCACACCAGGCCTGGGGTCCACCTCAAG GCCATGCCCCAACTGGTGGCTATGGTAGTGTCAACCCACCTCCATATATGCAACCACC ACCAAGACATGATAATTACTATCCTCCTCCTGAAATGCGTCCACCTACAATGGAGAAA CAGCCTCATCAAGGTATATCTGCATACGGAAGGGAGCCTCCTATGAATGTGCATGTAT CATCGGCCCCACCTATGTCCGCTCAGCAAGTTACGCAGCAAATGCAGATTCCACTGTC TTACGCAGATGCTGTGATTGGGACATCAGGTTCAAACATAAGCTACACTCGACGTCTT AGTGGAGCAACAGTAACCATTCAGGAAACCAGAGGAGTACCTGGTGAAATGACTGTTG AGGTTAGCGGAACTGGTTCACAAGTCCAGACCGCTATGCAGCTTATCCAGAACTTTAT GGCTGAAGCTGGAGCGCCGGCACCAGCACAGCCGCAAGCTGTAGCACCAGAGCAGCAA GGCTATAACCCCTATGCAACCCACGGGTCAGTCTATGCAACGGCTCCGACGAACCCGC CTGGAGGATACGCTACGGATTACAGCTCAGGCTATGGTTAC

TRINITY_DN40841_c0_g2 ATGGCCGAAGCTGAGGAACAACAAAATTTTGTCGCACACAATGGAGATGAGGTGCATA ATGGTTTACAGTACCAGGTCCATGACGAGACCCTTGCTCACCAACCATACGAGGTTCA AGACCCGATTCTTGAACCTCAGCAGTACGAGGTTCAAGATCCGACCCTTGAACCCCAG CAGTACGGTGGTCAATTAGAGTACCAAGACTTCCAGGTGCAGGACCAGGCTAATGAAG AAGTCCACGATCAGCCGCAAGATGAACTACAATATCAACCTCAAGAGCAGGAGCAGTA TCAGTTGCACGACCAGGCTCATGGTCAGGCGCAGTATCAGGTTGAAGGCGAGGCACAG GATCAGGATGGTGATGAAGTGCAAGATCAAGTTGACGGTGAGGAAGAAATTCCTGAGC ATGTTGAGTCTCTACAGAAATCAGAGCTTGAAAGTGATATAAGTATAACAGGCGGAGG TGAGGAGAAGCGATGGCCGGGGTGGCCCGGAGAAACAGTGTTCCGTATGCTGGTTCCT GCACAGAAAGTGGGTAGCATCATTGGTCGCAAAGGTGATGTCATTAAGAAAATAGTTG AGGAGACAAGGGCTCGGATAAAGATTCTTGATGGTCCTCCAGGCACAACCGAAAGAGC TGTTATGGTTTCTGGAAAAGAAGAGCCCGAATCATCTCTGCCTCCTTCTATGGATGGC CTTCTGAGAGTCCACATGCGGATAGTTGATGGTCTAGATGGTGAAGCTTCTCAGGCCC CTCCGCCTACAAAGGTTTCAACAAGATTACTAGTCCCAGCATCTCAGGCTGGAAGTTT GATTGGAAAACAAGGAGCAACAGTTAAAGCCATTCAAGAAGCATCTGGCTGTATAGTT AGAGTCCTCGGATCAGAGGATTTGCCTGTTTTTGCTCTTCAAGACGATAGGGTTGTTG AAGTTGTGGGGGAGCCAACAAGTGTTCATAAAGCCTTGGAGCTAATTGCATCACATCT CAGAAAGTTCTTGGTTGACCGTAGCATCATCCCATACTTTGAAAATCAGATGCAAAAG CCAACTCGCCAGATGGATCATATGCCGCCACCACACCAGGCCTGGGGTCCACCTCAAG GCCATGCCCCAACTGGTGGCTATGGTAGTGTCAACCCACCTCCATATATGCAACCACC ACCAAGACATGATAATTACTATCCTCCTCCTGAAATGCGTCCACCTACAATGGAGAAA CAGCCTCATCAAGGTATATCTGCATACGGAAGGGAGCCTCCTATGAATGTGCATGTAT CATCGGCCCCACCTATGTCCGCTCAGCAAGTTACGCAGCAAATGCAGATTCCACTGTC TTACGCAGATGCTGTGATTGGGACATCAGGTTCAAACATAAGCTACACTCGACGTCTT AGTGGAGCAACAGTAACCATTCAGGAAACCAGAGGAGTACCTGGTGAAATGACTGTTG AGGTTAGCGGAACTGGTTCACAAGTCCAGACCGCTATGCAGCTTATCCAGAACTTTAT GGCTGAAGCTGGAGCGCCGGCACCAGCACAGCCGCAAGCTGTAGCACCAGAGCAGCAA GGCTATAACCCCTATGCAACCCACGGGTCAGTCTATGCAACGGCTCCGACGAACCCGC CTGGAGGATACGCTACGGATTACAGCTCAGGCTATGGTTAC

TRINITY_DN40841_c0_g1

ATGGCCGAAGCTGAGGAACAACAAAATTTTGTCGCACACAATGGAGATGAGGTGCATA ATGGTTTACAGTACCAGGTCCATGACGAGACCCTTGCTCACCAACCATACGAGGTTCA AGACCCGATTCTTGAACCTCAGCAGTACGAGGTTCAAGATCCGACCCTTGAACCCCAG CAGTACGGTGGTCAATTAGAGTACCAAGACTTCCAGGTGCAGGACCAGGCTAATGAAG AAGTCCACGATCAGCCGCAAGATGAACTACAATATCAACCTCAAGAGCAGGAGCAGTA TCAGTTGCACGACCAGGCTCATGGTCAGGCGCAGTATCAGGTTGAAGGCGAGGCACAG GATCAGGATGGTGATGAAGTGCAAGATCAAGTTGACGGTGAGGAAGAAATTCCTGAGC ATGTTGAGTCTCTACAGAAATCAGAGCTTGAAAGTGATATAAGTATAACAGGCGGAGG TGAGGAGAAGCGATGGCCGGGGTGGCCCGGAGAAACAGTGTTCCGTATGCTGGTTCCT GCACAGAAAGTGGGTAGCATCATTGGTCGCAAAGGTGATGTCATTAAGAAAATAGTTG AGGAGACAAGGGCTCGGATAAAGATTCTTGATGGTCCTCCAGGCACAACCGAAAGAGC TGTTATGGTTTCTGGAAAAGAAGAGCCCGAATCATCTCTGCCTCCTTCTATGGATGGC

Continued next page 
Supplemental Table 2. Continued.

\begin{tabular}{ll}
\hline Gene ID & Coding sequence
\end{tabular}

CTTCTGAGAGTCCACATGCGGATAGTTGATGGTCTAGATGGTGAAGCTTCTCAGGCCC CTCCGCCTACAAAGGTTTCAACAAGATTACTAGTCCCAGCATCTCAGGCTGGAAGTTT GATTGGAAAACAAGGAGCAACAGTTAAAGCCATTCAAGAAGCATCTGGCTGTATAGTT AGAGTCCTCGGATCAGAGGATTTGCCTGTTTTTGCTCTTCAAGACGATAGGGTTGTTG AAGTTGTGGGGGAGCCAACAAGTGTTCATAAAGCCTTGGAGCTAATTGCATCACATCT CAGAAAGTTCTTGGTTGACCGTAGCATCATCCCATACTTTGAAAATCAGATGCAAAAG CCAACTCGCCAGATGGATCATATGCCGCCACCACACCAGGCCTGGGGTCCACCTCAAG GCCATGCCCCAACTGGTGGCTATGGTAGTGTCAACCCACCTCCATATATGCAACCACC ACCAAGACATGATAATTACTATCCTCCTCCTGAAATGCGTCCACCTACAATGGAGAAA CAGCCTCATCAAGGTATATCTGCATACGGAAGGGAGCCTCCTATGAATGTGCATGTAT CATCGGCCCCACCTATGTCCGCTCAGCAAGTTACGCAGCAAATGCAGATTCCACTGTC TTACGCAGATGCTGTGATTGGGACATCAGGTTCAAACATAAGCTACACTCGACGTCTT AGTGGAGCAACAGTAACCATTCAGGAAACCAGAGGAGTACCTGGTGAAATGACTGTTG AGGTTAGCGGAACTGGTTCACAAGTCCAGACCGCTATGCAGCTTATCCAGAACTTTAT GGCTGAAGCTGGAGCGCCGGCACCAGCACAGCCGCAAGCTGTAGCACCAGAGCAGCAA GGCTATAACCCCTATGCAACCCACGGGTCAGTCTATGCAACGGCTCCGACGAACCCGC CTGGAGGATACGCTACGGATTACAGCTCAGGCTATGGTTAC

TRINITY_DN70605_c0_g2

ATGGCGTCAGTTACGAAGCCGTTCAGAGCACGTGAATCCGAAGATGCCGGTTTCACAT CGAACAATCTATGGGTGGGTAGCCTGACGATGGATACGACGGAGTCAGATCTGACGGA GTTGTTTGGAAGGTTCGGCGATATCGACAGAATCACGGCGTACTCGTCCCGTGGCTTC GCCTTCATCTACTACAGACACGTCGAGGAAGCTGTCGCGGCGATGGAGGCCCTCCAAG GGGCGAATTTGAACGGAAGTCAGATTAAGATCGAATACGCGCGACCGGCAAAACCTTG TAAGAGTCTCTGGGTGGGCGGAATCAGCCCGAGTGTTTCAAAGGATGACCTGGAGGCA GAATTCAGTAAATTTGGAAGAATCGTGGATTTTAGGTTTCTTAGGGAACGCAAGACAG CTTTCATTGATTATTACGAAATGGAAGATGCCGTACAGGCAAGAAGCATGAGTGGCAA GCGAATGGGTGGTAGCTATTTGCGCGTTGATTTTCTCCGGTCACAAGCGCCCAGAAAA GAACAATGGGGTGGCTCTTACGATAACAGGAATGGCAATGTGAATCATAAACCGCAGT ATCCTCACTCGCATGAAGATGCCAAAGGAGATGACCAGCCAAGTAAGGTTCTGTGGAT TGGGTACCCTCCTTCTGTTCAGATAGATGAGCAGATGCTACACAATGCAATGATACTC TTTGGTGAGATCGAGAGGAAAAAAAGTTACCCGTCAAGGCATTTTTCACTTGTGGAGT TTAGGAGCGTGGAAGAAGCTCGCCAAGCCAAGGAAGGGCTACAGGGGAGGTTATTCAA AGATCCTAGAATCACAATTATGTACTCAAACGATGAGATACCTCCTGAGCAAGATGAT ACTAGTTTTTATTCTGGCGTGAAACGGTCAAGGCCAGATATGTTCATCAATGATCCTA CTGGGTCTGTGAGGCCCTTCAGAGGTAGCAATGAGCGTCCTTATAATGGTTCAGAATA CAGTGACGTTGCTGGTAAGGAGCCAAACTGGAGGAGGCCGTCTCCAAATGGAACTGGA ATACTCCCATCTCCAACGGGACATGGAATTCTCCCATCTCCTGCACAAGGTATGAGGA ACCCTATGAGGTCAAACCCCGGTTCATGGGAAGGATATGATCCTGCTCAGTTGGATAG AGAAAGCAAACGAACCAGAAGAGATGGATCGGTTGACGGTTTTACTCCAATGGGTGTG GATGAGAGGTCATTTGGGCGTGGTTCAGTTGCTGCTAGGCCTATCCGTGGTTACCCTG ATTCTGACTACATGTGGAGAGGAATGATTGCCAAGGGTGGAACTCCCGTCTGTTGTGC TCGTTGTGTACCTATTGGAAAGGGGATCGAAACTAAGCTCCCCGAGGTCGTCAATTGT TCAGCAAGAACTGGTCTGGATATGCTCGCCAAACATTACACCGAAGCCATTGGATTTG AGATAGTTTTCTTCTTACCAGACAGTGAAGAGGATTTTGCGTCTTACACTGAATTTCT CCGCTACCTTGGCTCGAAAAATCGAGCTGGTGTTGCTAAATTAGATGATGGAACAACT TTATTCTTGGTACCTCCTTCAGATTTTTTAACTGACATACTCAAAGTGTCCGGTCCAG AACGGCTATATGGTGTTGTTCTCAAGTTGCCTCCGCCAGCCGTTCCTGTCGCAGCAAC ATACAGACAAGAATCTCAATCCAATCCTCTGCCTTATATGGATCAAGCGCGGGATTCA CCTGCCAATGCCGGTCACAGTTTATATCCTCCTCGGGAAAATTACAATAGGGGTGCAC TAGAACAGTTGACAGCTCCTTCAAGACCATCGGTTAGCGAGCCACTCAGAATACCTAA CAATGCAGCGGCGTCTCAAGCTGGGGTTAGTTTAACTCCGGAGCTTTTAGCCACCCTG GCATCTATTCTCCCTGCAACTTCTCAATCTGCTGCTCCCGAGAGTCACCAACCTGTGT CAGGTACTTCAACAGTCGTTTCCACAGTACCTCAGTACAATGGAGAAGCACAGCCTCA AGCTTGGAATAGAGATCCGCAAACAGTGCATGATGCCTCAAATCAGTCTTTCCAACAA TATGGAAATCAGTACAATCCAGCCGGGCAACTACCTCCTCCTCCTGCGCGTTATCCTC CAGCTTCAAACAGCCCTAACCCCAACTACTCTAGTGGAATCGTCCGTGGCAACATGCA GTATCAAGGCCAAGGACAATCTGTTAACATGCCTCAGATGTCTCCGTTACCGAATATG CCTCAAAATAATTATGCCATGTACACTCAGGGTTCGTTAAATCATCCTGTTTCTCAGC

Continued next page

J. Amer. Soc. Hort. ScI. 143(1):1-38. 2018. 
Supplemental Table 2. Continued.

\begin{tabular}{|c|c|}
\hline Gene ID & Coding sequence \\
\hline & CCATGACGCAGCAATACCAACCAGAAGCTTCCGTACCAAACCAAAATTATGGTGCCGT \\
\hline & TCCAAGTTACCAGCAAGGTAACTATTATGGTTTAACAACAAATCAGGCGCATAATATA \\
\hline & AATCСТTCCCAATTTCAAGCTGCCATGCAACCACCAGCTGACAAGGCAAACTTAGAGC \\
\hline & CACAAAGCCAAGCAGCACAGCCTATGCTCTCTGGGGCTGGTCAGGGTACAACAAATGG \\
\hline & GGAGGTCGAGAAGGACGAGAGATATCAGAAAACACTACAGTTTGCAGCAAGCCTTCTT \\
\hline & CTCCAGATAAAGCAGAATCAGCAGCAGCCGCCTTCAGGTACTCCGGCTGGACAGGGGCCT \\
\hline \multirow[t]{6}{*}{ TRINITY_DN60601_c0_g2 } & ATGTCAGGAGTTTGGGTGTTCAACAACGGAGTGATACGTCTAGTAGAAAACCCAAACC \\
\hline & AGTCCGGTGGAGCGACCACTCAGACGCACGGCCGGAGGAATGTCTTGGTTTACTTGCC \\
\hline & TACGGGTGAAGTAGTCTCTTCTTACTCGTCACTGGAACAGATCCTAAGGAGTCTTGGG \\
\hline & TGGGAAAGATACTTCAATGGAGACTCCGATCTCATCCAATACCACAAACGTTCCTCTA \\
\hline & TCGACCTCATCTCCTTACCAAGAGACTTCTCCAAGTTCAACTCTGTTTACATGTACGA \\
\hline & CATCGTCGTCAAGAACCCTAATTCCTTCCACGTCCGTGATTTCAAT \\
\hline \multirow[t]{6}{*}{ TRINITY_DN47820_c0_g1 } & ATGTCAGGCGTGTGGGTGTTCAAGAACGGAGTGATAAGGCTTGTGGAGAATCCTAACC \\
\hline & AGTCAGGAGGCGACACACACAGCCGAAGGAAAGTGATGGTCTATTTACCGACAGGAGA \\
\hline & AGTGATCTCATCTTACTCGACGCTCGAGCAGATCCTTCGCAGTCTTGGGTGGGAGAGG \\
\hline & TACTTCGGTGGCGGCGACACAGATCTCCTCCAATTCCACAAACGCTCATCCATTGATC \\
\hline & TCATCTCTCTCCСTAAAGATTTCACCAAATTCAGCTCCGTTTACATGTACGATATCGT \\
\hline & CGTCAAGAACCCTAATTACTTCCACGTCCGGGACTCCAAT \\
\hline \multirow[t]{39}{*}{ TRINITY_DN67831_c2_g1 } & ATGTCGTCCGAGTTGAAACAGCTAATCGTCGTTGCTGAAGGCACTGCAGCGTTGGGTC \\
\hline & CTTATTGGCAAACCATCGTCTCCGACTATCTCGAGAAAATCATCAGGTCTTTCTGTGG \\
\hline & CAGTGAGGTAAACGGAGAGAGGAACCCTGTTTCTAATGTTGAGCTATCACTGGTCATC \\
\hline & TTCAATTCTCATGGTTCATATTGTGCTTGCTTGGTACAAAGGAGTGGCTGGACAAAAG \\
\hline & ATGTCGATATTTTCTTGCATTGGCTTTCGTCCATACAATTTGCCGGTGGTGGTTTTAA \\
\hline & TGAGGCTGCCACAGCTGAAGGGCTTGCCGAAGCATTGATGATGTTTTCTCСТCСTTCA \\
\hline & GGCCAAGCACAGCCAAGCAATGATCTGAAAAGGCACTGTATCCTAATCACAGCCAGCA \\
\hline & ATCCTCACTCGTTGCCAACACCTGTATATCGTCCAAAAGTGCAAAATGCGGAACGGAA \\
\hline & TGAAAATGGTGATGCGCATTCTGAAAGTCGTTTATCAGATGCCGAAACAGTGGCATCA \\
\hline & TATTTTTCTAGGTGCTCTGTTTCATTGTCTGTTGTGTGTCCAAAGCAGCTTCCGAAGA \\
\hline & TAAGAGCACTATACAATGCGGGAAAGCTCAATCCACAAAGTGCGGACTTGTCGATTGA \\
\hline & CACGGTTAAGAACGCATTCTATCTTGTCCTAATCTCGGAGAATTTTGTGGAGGCACGT \\
\hline & GCTGCCTTGAGTCATTCTGCTACAAATTTGCCACAGACCACCCAAAGCCCTGTGAAAG \\
\hline & TGGACAGGGCCACTGTTGCTCCATCTCTTCCAGTCACTGGGCAACCTCCAGCTCCTGT \\
\hline & GCCATCAGCTAATGGACCTATTCTTAATCGGCAACCAGTTTCTGTTGGACCAGTTCCA \\
\hline & ACTGCTACTGTGAAAGTTGAGCCTAGCACTGTACCATCTATGGCAGCAGTTCCAACTT \\
\hline & TTCCCCATATCCCCTCCGTAGCTCGGCCTGCTACACAAGCAAATCCTTCTATTCAAAC \\
\hline & ATCTACAGCATCTCCAATTTCTCAAGAAATGGTCACCAATGCCGAGAATGCACCAGAT \\
\hline & ATTAAGCCTGTGGTCGGTGGAATGACGCCACCATTGCGTACTGGTCCTCCTGGTGGAG \\
\hline & CTAACGTGAATCTGCTGAACAATCTTTCTCAAGTTCGACAAGTCATGAGCTCTGCAGC \\
\hline & TCTGGCGGGTGCAGCCTCGTCGGCTGGGCAAAGTGCGGTTGCAATGCATATGTCAAAT \\
\hline & ATGATATCAACAGGAATGGCTACATCTCTGCСTCСТTCACAAACTGTGTTTTCATCTG \\
\hline & GACAGCAGGGAATTACTTCAATGGTTGGTTCGGGTGCATTAATGGGAACTGCACAAGC \\
\hline & GGGACAAAGCCCAGGTCTTAATAATTCCTTTAGTCCTCAAACAACGTCAAATGTTGCT \\
\hline & TCAAACCTTGGTGTTTCTCAACCAATGCAAGGGGTGAACCAAGGAAGTCATTCTGGAG \\
\hline & CACAGATGATGCAATCCATGAACCAAAACATGATGAGTGGTCTCGGCCAAGGAAATGT \\
\hline & СТССТCTGGAACAGGTGGAATGATGCCTACTCCAGGAGTTGGCCAACAAGCGCAGCCA \\
\hline & GGAATACAACAACTCGGTGGCAGTAACAGTTCAGCTCCTAATATGCAGTTGTCACAGC \\
\hline & CATCATCGGGGGCTATGCAGACTCCTCAATCCAAATATGTTAAAGTCTGGGAGGGAAA \\
\hline & CTTATCTGGGCAAAGACAAGGGCAGCCTGTTCTTATCACCAGACTTGAGGGTTACCGA \\
\hline & AGCGCTTCTGCCTCTGATTCGTTGGCAGCCAATTGGCCACCGACTATGCAGATCGTTC \\
\hline & GTCTCATATCCCAGGATCATATGAATAACAAGCAATATGTTGGCAAAGCTGACTTCCT \\
\hline & TGTTTTTCGGGCCATGAGCCAACATGGGTTTTTGGGACAACTTCAGGATAAGAAGCTT \\
\hline & TGTGCAGTCATCCAGCTGCCATCACAGACGCTGCTTCTCTCCGTCTCTGACAAGGCTT \\
\hline & GCCGCTTGATTGGAATGCTTTTTCCAGGGGATATGGTTGTGTTTAAGCCGCAAATTCC \\
\hline & GAATCAGCAACAACAACAGCAACAACAGCTGCAGCAGCACCAACAACAGCAGCAGCAG \\
\hline & CAGCAACAGCAACAGATCCAGCAGCAGCAGCAGCAACAACAACACCACCACCAACAGC \\
\hline & AACAGCTGCCACAACTCCAGCAGCAGCAGCAGCATCAAATGTCACAGCTCCAACATCA \\
\hline & TCAGCAGCAACAGCAACAGCAGCATCAGTTGTCACAGCTCCAACATCATCACCAGCAG \\
\hline
\end{tabular}

Continued next page 
Supplemental Table 2. Continued.

\begin{tabular}{ll}
\hline Gene ID & Coding sequence
\end{tabular}

CAGCAGCAGACGTCACCGCTGAATCAGATGCAGCAACAGACTTCGCCACTGAATCAGA

TGCAGCAGCAGCAGCAGCAGCCTCAACAGCTAGTTGGGTCAGGAGTGATGGGTGGTCA

AACTTTTGCACAAGCTCCTGGAAGATCTCAACCAGGCGGCGGTGGGCAGCCTACCATG CCGGGAGCTGGCTTCATGGGA

TRINITY_DN67682_c0_g1

ATGGCTACTACTTCATCTGAGAGGTGGACCGATGGTCTTCAGTTCTCCTCCTTGTTAT GGCCTCCGCCACGAGACCCTCAACAACATAAGGATCAAGTCGTTGCTTACGTCGAGTA CTTTGGTCAATTCACATCAGAGCAATTCCCTGATGACATTGCTGAGTTGGTCCGCCAT CAGTATCCATCAACCGAGAAGCGCCTTTTGGATGATGTTCTGGCAATGTTTGTCCTTC ATCATCCGGAGCATGGTCATGCGGTTATCCTTCCAATTATTTCATGTCTCATTGATGG CTCTCTGGTGTACAGCAAGGACGCTCATCCCTTTGCCTCTTTCATATCGTTAGTTTGC CCAAGTAGTGAGAATGACTATTCGGAGCAATGGGCTTTGGCGTGCGGAGAAATCCTCC GCATTTTGACTCATTACAATCGTCCCATATATAAGACCGAGCACCAAAATGGAGAAAC AGAGAGAAATTGCCTGAGCAAAGCTTCGACTAGTGGGTCTACGCTTTCAGAGCCTAAG GTTGTATCACCGGTACAGCATGAAAGAAAACCGTTAAGGCCTTTGTCTCCATGGATCA GTGATATACTACTTGCTGCGCCCTTGGGTATAAGAAGTGACTACTTTCGTTGGTGTAG TGGGGTTATGGGTAAATATGCTGCTGGAGAGCTCAAGCCGCCAACCATTGCTTCTCGA GGATCTGGTAAACATCCTCAATTGATGCCTTCAACACCAAGATGGGCGGTTGCTAATG GAGCTGGTGTCATACTGAGTGTTTGCGATGATGAAGTTGCTCGATATGAGACTGCTAC GCTAACAGCGGTTGCTGTCCCTGCACTTCTTCTTCCTCCCCCCACGACATCCTTAGAT GAGCATTTAGTTGCTGGCCTTCCAGCTCTTGAGCCATACGCACGTTTGTTTCATAGAT ATTATGCCATTGCAACTCCAAGTGCTACTCAGAGACTTCTTCTTGGACTTTTAGAAGC CCCGCCGTCGTGGGCTCCAGATGCACTTGATGCTGCTGTACAGCTTGTGGAACTCCTT CGAGCTGCTGAAGATTATGCATCTGGTGTAAGGCTACCCAGGAACTGGATGCATTTGC ACTTCTTGCGTGCAATAGGAATTGCTATGTCTATGAGGGCAGGTGTTGCTGCTGATGC TGCAGCCGCTTTGCTTTTCCGCATACTCTCACAGCCGGCACTGCTTTTTCCTCCGCTA AGCCAAGTTGAGGGAGTAGAAATTCAACACGCGCCTATTGGTGGCTACAGTTCAAATT ACAGAAAGCAGATAGAAGTTCCTGCAGCAGAAGCAACCATTGAAGCCACTGCTCAAGG AATTGCCTCAATGCTTTGTGCGCATGGTCCTGAAGTCGAGTGGAGAATTTGCACTATA TGGGAAGCTGCCTATGGATTGATTCCTTTAAATTCCTCAGCGGTTGATCTCCCCGAAA TCATTGTCGCTACCCCACTGCAACTTCCTATCTTGTCATGGAATCTATACATTCCACT CCTGAAAGTACTTGAATATCTTCCACGGGGGAGTCCTTCGGAAGCATGCTTGATGAAA ATATTTGTCGCCACCGTGGAAACAATCCTCAGTAGAACTTTTCCGCCAGAAATTTCCG GGGAACATATCAGGAAAGCCAGATCGAGTTTTACCGCGAGATCAGCGACCAAAAACCT TGCTATGGCTGAGCTTCGTGCTATGGTCCATGCTCTCTTTTTAGAATCATGCGCTGGT GTTGAGATAGCGTCACGCCTACTTTTTGTTGTTTTGACTGTATGTGTTAGCCATGAAG CACAGTCTAGTGGGAGCAAGAGACCGAGAAGCGAATATGCTAGTACTGCAGAGGAGAA TCAATCTGTATCTGACAATCAAACTAGTAACCGTAAAAGTAGGAATGTCAAGGGACAG GGACCTGTGGCAGCATTTGATTCATACGTTCTCGCTGCTGTTTGTGCTCTTGCTTGTG AGGTTCAGCTGTATCCTATGATCTCTGGAGGGGGAAACTTTTCCAATACTGCAGTTGC TGCGACGATTCCGAAGTCTGTAAAAATAAATGGGTCATCTAACGAGTATGGAGCTGGG ATTGATTCGGCAATTAATCATACACGCCGAATCTTGGCAATTCTAGAGGCACTCTTTT CACTGAAACCGTCTTCTGTGGGGACTCCATGGAGCTACAGTTCTAACGAGATAGTTGC TGCGGCCATGGTTGCAGCTCATATCTCCGAATTGTTTAGACGTTCAAAGGCTTTGACG CATGCGTTGTCTGGGTTGATGAGATGTAAGTGGGACAAGGAAATTCACAAAAGAGCAT CGTCCTTGTATAACCTCATAGATGTTCATAGCAAAGTCGTAGCATCAATTGTCGACAA AGCTGAACCCTTGGAAGCATATCTTAAGAATGCACCGGTCCAGAAGGATTCTCTGACA TGTCTTAACTGGAAACAGAACACGTGTGCAAGCAGCGCAGGCTTTGGTACAGCAGTGA CGTCCACGTCACGTAGTGAAATGACTCCAAGAGGCAACCATAAGTATGCTAGACATTC AGATGAAGGCTCAGGGACTAGACCCTCAGAGAAGGGTATCAAAGATTTCCTCTTGGAT GCTTCTGATCTAGCGAATTTCCTCACAGCTGATAGGCTCGCGGGGTTTTATTGTGGTA CACAAGTTCTTTTGAGGTCAGTACTTGCAAAGAAACCGGAGCTTTCTTTCTCCGTTGT TTCACTGTTATGGCACAAACTGATCGCTGCTCCGGAAATCCAGCCCACAGCGGAAAGC ACCTCTGCTCAACAAGGATGGAGACAGGTAGTTGATGCACTATGCAACGTCGTATCTG CAACGCCAGCAAAAGCAGCTGCAGCAGTTGTTCTTCAGGCTGAGAGAGAGTTGCAGCC TTGGATCGCCAAAGATGATGAAGAAGGTCAGAAAATGTGGAAAATCAACCAACGGATA GTGAAAGTGATGGTGGAGCTGATGCGAGATCATGAGAGGCCAGAGTCACTGGTGATTT TGGCAAGTGCATCGGATCTTCTTCTGAGGGCAACTGATGGAATGCTTGTTGATGGAGA 
Supplemental Table 2. Continued.

\begin{tabular}{ll}
\hline Gene ID & Coding sequence
\end{tabular}

AGCTTGCACATTACCTCAACTTGAGCTACTTGAAGCCACGGCAAGGGCAATACAGCCG GTGCTAGCTTGGGGGACATCTGGACTAGCAGTGGTTGACGGTTTATCCAATCTATTGA AGTGTCGTCTACCGGCAACAATACGGTGCCTTTCACACCCAAGTGCACACGTACGTGC CTTGAGCACTTCAGTACTACGCGACATCATGAACCAAAGCTCCATAACCATCAAGGTA ACTCCAAAACCGCCGCCAACAACAGAGAAGAACGGGATGCACAGCCCGTCCTATCGGT TCTTCAACGCGGCCACCATAGACTGGAAAGCAGACATCCAGAAATGTTTGAACTGGGA AGCTCACAGCTTGCTCTCAACGACCATGCCTACTCAGTTTCTTGACACCGCGGCTCGA GAACTAGGCTGTACCATATCGTTGTCGTCCCAA

TRINITY_DN84722_c0_g1

ATGTCACGAACAGAAAATTCGTGTAAAATGTTTATGGCTTGTCTTGTCTCAAAGGTTA TACTTTTATGCTCTAGTATATTGAATGGTCGGTCTTGTGTTATTAACAGGCTGAGAGA GAGTTGCAGCCTTGGATCGCCAAAGATGATGAAGAAGGTCAGAAAATGTGGAAAATCA ACCAAC

TRINITY_DN69595_c0_g1

ATGGCTCCTGGGCGTAAAAGAGGAGCGAGCAAGGCCAAGGACAAGGGGCAGTTGATTC TCGGTGATCTCGTTCTTGCCAAAGTCAAAGGCTTCCCTGCTTGGCCTGCTAAGATAAG CCGGCCTGAAGAGTGGGATCGTGCTCCAGACCCGAAGAAGTACTTTGTTCAGTTCTTT GGAACACAGGAAATAGCTTTTGTTGCACCGCCTGATATCCAAGCCTTCACTAGTGAGG CAAAGAGCAAACTATTAGCAAGATGTCAAGGTAAAACAGTAAAGTATTTTGCACAAGC TGTAACGGAAATATGCACCGCATTTGAAGAGTTGCAAAACCACAAGTCTAATGTTTTG GGCAATGGGGATCCTCCCGATGCTACGGAGCCTGGGTTGACAAAGGCTAAGATAGTAG ATGGAATAGATCATATAGTCACTGATTCTGATGGAACTGATAACGGTGATTCTAGGGC GGATCCTTGCGTCCCCAAATTAGAAAAGAATAGTGGTGAAGAGACAGAGGCTGAGACA GGGAAGCAGGAATCATCTCCGTTTCGGGAGTCAAAGATCACAACCACATCCTCGGGTA GCGAGTCATTGGAGCATGGTTCATATGACTCTAAAATAAAGGAAGAAGATATTGATAA AGGAACCGACGGCGATGATTGCATTGATGGTCAAAAGAATCTGGCCAACGGTAAGGGA ATAAAGAAGGTAGCAGGTGGCTCGGACAAAAAAGGTGATACAGTTCATATAGAAAAAA GCAGTAACTCACATGTGCATGATGGTCGAGCTGCTAGTGGTAAATCTGATTCCAAGAA GTCAAAAGGTTTGTTGACAGAGAAATCTAGTTCCAAAGTGTCTGGAGGTAAGCGTGAA AGTTCGCCTGGCGTTAAGGGTGGTCTTTCCGGCAAGAAAAGGAGGCTTGAAAGTGAAC TAGGGAAGCATGCACCAAGAGTAGAAAAAAGCTCACCTGCTTCTAAAAAGCGGCGATG TGATGGTAAAACAGACAAAGGAAAGTGTGAGATTGATGATGAATCTGATTGTACTGAG GCTGTGTCAGATATTAAGCGTGAAATTGTTTTAGGAGTAAGTGCTCGTGGAGGAAATT TACAATATGATAAAGAAGTAGTTGCATATACAAAGCGCCGCAGACAAGCAGTTGAGCA TGATACCTCTCCTCCTTTTTCTGGGTCTCGGGATAAATCCGGGAAAGGTCAACTAGAA CAGAAAAACCGTTCATCTTCTATTAGTGATAAATCCGGG

TRINITY_DN69302_c0_g3 ATGTGTTTCCTGGTAGATTCATCCGCAGAAGTTAGCAACAGTTTTGGGCCCTGCGACGATTT TAGCCTCCTGCCGTTCTCTGACCTTCCATTTGAGAGACTAAGAATATCTTCCTCTGTT TCAGAAGCAATTGACTCTGCGAATCAACTTGCGAAATGTTACGATTTTGTTTCTTCA

TRINITY_DN69302_c0_g1 ATGAAGAAACAAAATCGTAACATTTCGCAAGTTGATTCGCAGAGTCAATTGCTTCTGA AACAGAGTATTGCTGAAATCATGGGTGATAGTAGCAATCCTGAAGATATTCTTAGTCT CTCAAATGGAAGGTCAGAGAACGGCAGGAGGCTTAAATCGTCGCAGGGCCCAAAACTG TTGCTAACTTCTGCGGATGATTCCACCAGGAAACACATGCTTGGTTCAACTCCATCAT ATAATAAAGAACGCAGGAAAGTTCAGATGGTGGAGCAACCAGGCCAAAAAGCTGCTGG AAGAAGCCCTCAGACAGTTAGAATAGGAACTTCAAGTCGAAGCCGCCCTATGTCTGCT GATGATATTCAGAAAGCGAAGATGCGTGCTCTGTATATGCATAGCAAGAATAGTAGAA AGGATCCCTTATCAAGTGCGATTAGTGATTCGAGAACCATTATTCCTGAGAAGCCCTT GGCTCTTCTGTCAGCCAAGGATTCTCCACCTAGTGAGAACAATGAAGCTAAGACTGAA GATACACCTGAACCTTCTGCTGTACAGCCTGTCAATTCACCGCCTGTAAATCTCCCGG TTCAGCCTGTCAATTCACCGGTTGTAGATGTCCCGGTTCAGCCTGTCAGTTCTCCGGC TGTAAATGTGCCGGTACAAGCTGATGAATTTAGAAAACCTTCAACACCTCCTAAAAGC ATTTCTAGTAAGGTGGGAATCTTGTTGAAAATGAGCCCACAAACTATTCTAAAGAATT GCAAGAAAAAACAGACTGATTGGCATGTACCACCAGAAATGAGACTTGACGACCTCTG GAGAGTAGCTGCTGGTGGTAATAGCAAGGAGGCAGATGTTCAGAAAAACCGAAACCGG AGAGAAAAAGAAACAACATATCAATCTCTTCAAACTATACCGTTGAATCCTAAGGAAC CATGGGACAGGGAACTGGACTTTGATGACAGTTTGACACCTGAAATCCCGTCTCAGCA GCCACCAGAAGAAAGTATAGCAGAACCAGAGGAATCACTTGATGATCGGCGAACTGCT GCTGGTGCTGCCACAACCTCTTCATCTCAAAGTAGTACCATGGAACCTGATTTTGAGT TATTAGCCGCCTTACTTAAGAACCCGGATCTTCTATATGCGCTGACTTCTGGTAATCC

Continued next page 
Supplemental Table 2. Continued.

\begin{tabular}{|c|c|}
\hline Gene ID & Coding sequence \\
\hline & $\begin{array}{l}\text { CGGTAATTTAGCCGGCCAAGATATGGTAAAACTGCTTGATGTGATTAAAACTGGTGCA } \\
\text { CCAAATTCAAGCAGTAGCTCATATAAGAAGGTTGAAGAAAAGGTTGAAGTTTCCCTTC } \\
\text { CATCTCCCACTCCATCAACCAATCCTGGAATGAGCGGGTGGGGACAAGAAGTGATTCG } \\
\text { GAATCCATTTCAAGGCAAAACCAAGTTGGTGCGGCAGCTGCTAGAATGAGTACTCAG } \\
\text { CTTGCCACTCCAATGCAGTGGCAATCATCAAACGAACAATCGATTCCTCGACATGCTC } \\
\text { CATCAGCATATAACTCTCTCACATTGCCTCGTACAGAAAGACAAGTACAACAACAACA } \\
\text { ACCTATGCAACCAAGACTGCTTCATCAGAGTCAACATCTTCAACAACAACAACCAATG } \\
\text { CCGACAAACTCGTACGCGGTTAGGGAATCAGTAGGACAAATGGGCACAGGTACATCGG } \\
\text { CTTCATGGAGGTCCCAGCAGAGTCAGAATAGTTACTACACACCTCAAGCAAACGGGAT } \\
\text { TGCGGCGGCTTCTTCTTACCAAGTGAGTGAGCAGTACATGAGTAGTGAACCAGGATTT } \\
\text { GAATCATGGAGTCCTGATCATAGCCCAAGTAGGAACCACACTAATATGAGGGGACAAC } \\
\text { AACAACAACCGCCATCTAGGAAACATGATTATTCATCTTCTCATCCTTATTGGAACCA } \\
\text { AAACAGAAGATGGCGT }\end{array}$ \\
\hline TRINITY_DN69302_c0_g2 & $\begin{array}{l}\text { ATGTGTTTCCTGGTAGATTCATCCGCAGAAGTTAGCAACAGTTTTGGGCCCTGCGACG } \\
\text { ATTTTAGCCTCCTGCCGTTCTCTGACCTTCCATTTGAGAGACTAAGAATATCTTCCTG } \\
\text { TTACAACACGAAAAAAGGTTTAATAGAACTGATGTGGAGCACC }\end{array}$ \\
\hline TRINITY_DN68323_c1_g1 & $\begin{array}{l}\text { ATGGATACAAATACATCTGGAGGAGAAGAACTATTAACTAAGGCAAGAAAGCCATATA } \\
\text { CGATTACAAAGCAGCGGGAACGATGGACAGAGGATGAGCATGATAGGTTTCTAGAAGC } \\
\text { CCTGAGGCTTTATGGAAGAGCTTGGCAACGTATTGAAGAACATATTGGGACAAAGACT } \\
\text { GCTGTTCAGATCAGAAGTCATGCTCAAAAGTTCTTCACAAAGTTGGAGAAAGAAGCTG } \\
\text { AAGCTAAAGGCATTCCTGTTTGTCAAGCTCTGGACATCGAAATTCCACCTCCTCGTCC } \\
\text { CAAAAGAAAACCCAATACTCCTTATCCTCGGAAGCCTGGCAATAACGGTCAATCGTCC } \\
\text { TCACAAGTATCATCAGCAAAAGATGCAAAACTTGTATCGTCGGCCTCTCTTCACAAT } \\
\text { GTAACCAGGCGTTCTTGAATCTGGAGGAAGTGCCGATTTCTGAGAAAACATCAACTGG } \\
\text { GAAAGAGAATCAAGATGATAACTGCTCAGGGGTTTCCACTGTGAACAAGTATCCCTTA } \\
\text { CCAAAAAAAGTAACTCCAGGGAACCGAAAGACGTTCATGGAATCTGACCCGTCTCTAA } \\
\text { GAAAGGCAAGTGCTGATAATGAAACAAGTAAGGACTCAAATGTAGACAACATGGTTCA } \\
\text { AGATGTTCCCGAGAAGAGCAAAGACAGAGATGGTGACGGCATGCACAGCGCAAGGAAC } \\
\text { TACCCTTGGCATTTCCCGGCGGATTTTGTAAACGGAAATATGGCAAAATGCTCTCAAT } \\
\text { TCCATCCCCCAGGTATGGTATCTCAAGACTTCATGTTTTGTCCTATGGGAGATCGAGT } \\
\text { TCACGGGCACTCTAGTTCGCACGTGAATCTTCCAGCTACAACAACATCATCTGCGACT } \\
\text { ACTACTACAGCTTCTCAGCAAGCGTTTCCAGCGTGCCATTCCCAGGATGATTTCCTT } \\
\text { CGTATCTCCAGATCTCTTCTACTTTCTCCAATCTTATTATGTCAACTCTCCTACAGAA } \\
\text { TCCTGCAGCTCATGCTGCAGCCACATTCGCTGCTTCTGTCTGGCCTTATACGAATGTC } \\
\text { GGTAATTCGGGAGATTCATCAACGCAAATGAACTCTTCTCCTCCAAGTATAGCTGCCA } \\
\text { TCGCTGCTGCTACAGTAGCTGCTGCAACTGCTTGGTGGGCTTCTCATGGACTTCTTCC } \\
\text { TGTATGTGCTCCTACTCCAGTCGCATGCCTTCCATTACCAACCGTTGCAGTTCCAACT } \\
\text { CCAGCGATGGATAAGATGGATACCGTTGAAAATGATCAACTACCGCTTGAGAAACAAA } \\
\text { ACACAGCTCTGCAAGATCAAAACCTGGCCTCAAAATCTCCAGCTTCATCATCCGACGA } \\
\text { TTCAGAAGAGACTGGAATAACCAAGCTAAACGCTGACTCAAAGACAAATGCTGACAAG } \\
\text { GAGGAAGTTGTTGTTGGTGCTGCTGCTTTGCATGATTCAAACGTTTCCCAGAACAAAA } \\
\text { AGTTGGTGGACCGCTCATCGTGTGGGTCAAACACACCTTCAGGGAGCGACGCGGAAAC } \\
\text { AGACGCTTTAGATAAAATGGAGAAAGATAAAGAGGATGTCAAGGATGTCAAGGAGGCA } \\
\text { GATGCAAATCAGCCAGGTGTTATTGAGTAAGTAACCGTCGGAGTAAAATCAGAGACA } \\
\text { CCAACAACCAAACTACTGATTCATGGAAGGAAGTCTCCCAAGGGGGTCGTATAGCGTT } \\
\text { TCAGGCTCTCTTTGCAAGAGAGAGACTGCCTCAGAGCTTTTCACCTCCTCAAGTGGCA } \\
\text { GAGAATGTGAATGGAAAACAAAGTGACACTTCAATGCCAATGGCTCCTGATTCAACA } \\
\text { AAATTCAAGAATCTTGTGATGCAGACCAAGAAAGTGTAGTAATGATCGGTGCTGGACC } \\
\text { GGGGAAGAGCCTCAAAACGAGACAAACAGGGTTTAAGCCATACAAGAGATGTTCAATG } \\
\text { GAAGTGAAAGAGAGCCAAGTTGGGAACACAAACAATCAAAGCGATGAAAAAGTCTGCA } \\
\text { AGAGGCTTCGATTGGAAGGAGAAGCGTCAACA }\end{array}$ \\
\hline TRINITY_DN63261_c1_g1 & $\begin{array}{l}\text { ATGCAAAATCAAATGGAGTGGGATAGTGATTCCGATCTCAGCGGCGGAGATGAGGTAG } \\
\text { CTGAAGATGGTGAATGGTTCGGCGGAGATGACGGACCGATTCCGTTCCCGGTGACTAG } \\
\text { TCTTCCTGGAACTGGGCCGTGTGGGTTCGTGGTCAGCGACGCTCTTGAGCCTGACCAA } \\
\text { CCTATCATTTATGTTAACACCGTCTTCGAGATTGTCACTGGGTTTCGAGCTGAGGAAG } \\
\text { TTATTGGTAGAAATTGTCGGTTCTTGCAGTGTAGAGGTCCGTATGCTAAAAGAAGGCA }\end{array}$ \\
\hline
\end{tabular}


Supplemental Table 2. Continued.

\begin{tabular}{ll}
\hline Gene ID & Coding sequence
\end{tabular}

TCCATCAGTAGATTCTACAGTTGTCTCGAAGATGCGAGAATGTCTTGAAAAAGGCATC GAGTTTCAAGGCGAGTTGCTTAACTTCCGAAAAGATGGGTCTCCTTTTATGAACAAGC TGCGTCTAGTCCCTATCCGTGAAGAAGAAGAGATCACTCATTTCATAGGTGTTCTCTC CTTCACAGACGCAAAAATCGATCTCGGCCCATTTCCTGAGTTATCTACAAAAGGAATC TCAAGAAGGCCTCGCTCATTTTCCTCTGCGTTACCAATCGGAGAGCGTAATGTTTCTC GTGGACTATGTGGGATATTCGAGCTGAGTGATGAGGTAATAGCTCTCAAGATATTGTC AGAGTTGACTCCGCGTGATATTGCATCGGTTGGTTGTGTGTGTCGGCGGCTCAATGAG TTGACAAAGAATGATGATGTATGGAGAATGGTCTGCCAAAACACGTGGGGGACCGAGG CTTCACGTGTTCTGGAGAGTGTTCCTGGTGCAAAGAGGATCGGTTGGGTGCGACTGGC CCGAGAGTTTACCACACATGAAGCAACGGCATGGAGGAAATTTTCGGTTGGAGGTACT GTTGAGCCTTCCCGGTGTAATTTCAGCGCATGTGCGGTTGGGAACAGGATTGTTATCT TTGGTGGGGAAGGTGTGAATATGCAACCGATGAATGATACATTTGTGTTGGACCTTGG CTCGAGTAGTCCCGAGTGGAAGTCCGTTCTGGTTAGCTCTCCTCCTCCTGGTCGCTGG GGTCACACGCTTTCTTGTGTCAATGGTTCTCGTTTAGTAGTCTTTGGAGGTTACGGGA GCCACGGATTGCTCAACGATGTCTTCTTATTAGACCTTGATGCAGACCCTCCGACATG GAGAGAAGTATCCGGTTTGGCTCCTCCAATACCAAGATCGTGGCATAGCTCTTGCACA CTCGATGGAACCAAGCTGATTGTATCTGGTGGTTGTGCTGATTCAGGAGCTCTACTCA GCGATACATTCCTGCTTGACCTGTCGATGGATATACCAACATGGAGGGAGATACCGGT TCCGTGGTCTCCTCCATCTCGCCTTGGGCATACTTTAACCGTCTATGGTGACCGCAAG ATCCTCATGTTTGGTGGTCTTGCTAAGTCCGGTTCTTTGAGGTTCCGCTCTAACGACG TATACACGATGGATCTCAGCGAGGATGAACCGTGCTGGAGACCTGTGATTGGTTATGG ATCTAGCCTTCCCGGAGGCATGGCGGCTCCACGACCGAGGCTGGATCATGTGGCGATT AGCCTTCCCGGTGGTAGAATCTTGATCTTTGGTGGTTCGGTTGCAGGGATTGACTCGG CTGCTCAGCTTTATCTTCTTGATCCAACGGAGGAGAAACCAGCATGGAGAATACTGAA CGTTAAAGGAAGCCCACCGCGGTTTGCGTGGGGACACACCACTTGTGTGGTCGGAGGA ACTAGGTTGGTCGTGTTAGGTGGCCAAACCGGAGAAGAGTGGATGCTAAATGAAGCTC ATGAACTGTTATTAGCTACCTCTACCACTACAATCACCTCACCAAGACATGAAGAGAA GAGGGTCTTT

TRINITY_DN31883_c0_g1 ATGATCCTAGCACTGTGTCTTGACCGCCTCGAGCCCTCAGAGGCCTGGCTCGGCCTAG ACCCTGACATGTTTTTCCTAGCTAAACCCAGAAACACGACGCAACTGCTGCAGGAATC ATCCTCCCGTGTTCCACTATTCAATGATTCATCATTCACAAAGCAACAAGCAACTGAA CCACTT

TRINITY_DN76871_c0_g1 ATGTCAGGGTCTAGGCCGAGCCAGGCCTCTGAGGGCTCGAGGCGGTCAAGACACAGTG CTAGGATCATTGCCCAGACCACTGTTGATGCAAAACTCCATGCTGATTTTGAGGAGTC AGGCAGTTCCTTTGATTACTCAACGTCAGTGCGTGTTACTGGCCCGGTTGCGGAGAAC CAGCCACCGAGGTCTGACAAGGTGACCACGACTTATCTTCATCACATACAGAAGGGAA AGCTGATTCAGCCCTTCGGTTGTTTGCTCGCCTTGGATGAGAAGACCTTCAAAGTCAT TGCATACAGCGAGAATGCTCCTGAGCTGTTGACAATGACCAGTCATGCAGTTCCTAGT GTTGGCGAGCACCCTGTTCTAGGCATTGGGACGGATATAAGGAGCCTTTTCACTGCTC CTAGTGCCTCTGCATTGCAGAAAGCCCTTGGATTTGGAGATGTCTCCCTGTTGAATCC CATTCTTGTGCACTGCAAGACTTCTGCAAAGCCCTTTTATGCGATCGTACACAGGGTT ACAGGGAGCATCATTGTCGACTTTGAACCGGTGAAGCCTTATGAAGTTCCCATGACAG CTGCTGGTGCTTTGCAATCATACAAACTCGCTGCGAAAGCAATCACTAGGCTGCAATC TTTGCCCAGCGGGAGCATGGAAAGGCTTTGCGATACGATGGTTCAGGAGGTTTTTGAA CTGACGGGGTATGACAGGGTGATGGCTTATAAATTTCATGACGATGATCATGGTGAGG TTGTCTCTGAGGTTACAAAACCTGGACTGGAGCCTTATCTTGGGCTGCATTATCCAGC CACCGACATTCCTCAAGCAGCCCGTTTTCTGTTTATGAAGAACAAGGTTAGGATGATC GTCGATTGCAATGCAAAACATGTTAGGGTGCTTCAAGATGAAAAGCTTTCCTTTGATC TTACCTTGTGTGGCTCCACCCTTAGGGCACCACACAGCTGCCATTTGCAGTACATGGC CAACATGGATTCAATAGCATCTCTGGTGATGGCGGTTGTAGTAAACGAGGAAGATGGA GAAGGGGATGTCCCTGATGCTACTACACCTCAAAAGAGAAAGAGGCTATGGGGTTTGG TGGTTTGTCATAACACGAGTCCGAGGTTTGTTCCGTTTCCTCTCAGGTATGCCTGTGA GTTTCTAGCTCAAGTGTTTGCGATACACGTCAATAAGGAGGTAGAATTGGAGAATCAG ATTGTGGAGAAGAACATTTTGCGCACACAGACGCTCTTGTGCGATATGCTGATGCGTG ATGCTCCGCTGGGTATTGTGACACAGAGCCCCAACATAATGGACCTTGTGAAATGTGA TGGAGCAGCTCTCTTGTATAAAGACAAGGTATGGAAGCTGGGAGTAACTCCAAGTGAG TTCCATCTGCAAGAAATAGCTTCATGGTTGTGTGAATACCACACGGACTCAACGGGTC

Continued next page 
Supplemental Table 2. Continued.

\begin{tabular}{ll}
\hline Gene ID & Coding sequence
\end{tabular}

TGAGCACTGATAGCTTGCATGACGCTGGATTTCCTAGGGCTCTAGCTCTTGGGGATTC AGTATGTGGAATGGCGGCCGTGAGGATATCATCGACAGACATGATTTTCTGGTTCCGT TCTCATACCGCTGGTGAAGTGAGATGGGGAGGTGCAAAGCATGACCCAGATGATAGGG ATGATGCAAGGAGAATGCACCCGAGGTCATCTTTCAAGGCTTTCCTTGAAGTGGTCAA GACAAGGAGTTTACCTTGGAAGGACTATGAGATGGATGCCATACACTCCTTGCAGCTT ATTCTGAGAAATGCTTTCAAGGATGGTGAAGCTACTGATGTGAATACAAAGATCATCC ACTCGAAGCTTAATGATCTAAAAATTGATGGTATACAGGAGCTAGAAGCTGTGACCAG TGAGATGGTTCGTTTAATTGAGACTGCTACGGTGCCAATATTGGCGGTTGATTCTGAT GGATTGGTTAATGGTTGGAACACGAAAATTGCTGAGCTGACTGGTCTTCCGGTTGATG AAGCAATCGGGAAGCATCTCCTCACACTTGTTGAAGATTCTTCAGTGAAAATCGTTAA GAGGATGTTAGAGAATGCATTAGAAGGAACCGAGGAGCAGAACGTCCAATTTGAGATC AAGACGCATCTATCAAGAGCTGATGCTGGACCAATAAGTTTAGTTGTAAATGCATGCG CAAGTAGAGATCTCCATGAAAACGTGGTTGGGGTGTGTTTTGTAGCCCATGATCTTAC TGGACAGAAGACTGTGATGGACAAATTTACTCGGATTGAAGGTGATTACAAGGCGATC ATCCAGAATCCAAACCCGCTGATCCCGCCTATATTTGGTACCGACGAGTTCGGGTGGT GCACGGAGTGGAATCCGGCAATGTCAAAGTTAACCGGTTTAAAGCGAGAGGAAGTGAT GGACAAGATGCTGTTAGGAGAAGTCTTTGGTACGCAAAAGTCATGTTGTCGTCTAAAG AATCAAGAAGCATTTGTGAACCTTGGGATTGTGCTAAACAATGCTGTGACCAGTCAGG ATGCAGAGAAAGTGTCCTTTGCTTTCTTTACAAGAGGTGGAAAGTATGTGGAGTGTCT CTTGTGTGTGAGTAAGAAACTGGACAGGGAAGGTGTAGTGACCGGCGTCTTCTGTTTC CTGCAACTTGCCAGCCACGAGCTGCAGCAAGCACTCCATGTTCAGCGTCTAGCTGAGC GAACTGCATTGAAGAGATTGAAGGCTCTAGCTTACATAAAAAGACAGATCAGGAATCC GCTATCCGGGATCATGTTTACACGAAAAATGATGGAAGGTACTGAGTTAGGACCGGAG CAGAGACGGATTTTGCAAACTAGCGCTTTATGTCAGAAGCAACTCAGCAAGATCCTGG ATGATTCCGATCTCGAAAGCATCATTGAAGGGTGCTTGGATCTAGAAATGAAAGAATT CCGCTTAAACGAAGTGTTGACTGCTTCCACAAGTCAAGTAATGATGAAGAGTAACGGA AAGAGCGTTCGGATAACAAATGATACCGGAGAAGAAGTAATGTCTGATACTTTGTATG GAGACAGTATCAGGCTTCAACAAGTCTTGGCAGACTTCATGCTGATGTCTGTTAACTT TACACCATCCGGAGGCGAGCTAACTGTTACAGCTTCCTTGAGGAAAGATCAGCTCGGG CGATCTGTGCATCTTGCTTACTTAGAGATAAGAATAACGCATACCGGAGCTGGGTTAC CAGAGTTTTTGCTAAACCAAATGTTTGGAACTGAGGAAGATGTGTCGGAGGAAGGATT AAGCTTGATGGTTAGCCGGAAACTGGTGAAGCTGATGAATGGAGATGTTCAGTACCTG AGACAAGCTGGGAAATCGAGTTTCATTATCACTGCAGAACTCGCTGCAGCAAACAAG

TRINITY_DN76871_c0_g2 ATGTCAGGGTCTAGGCCGAGCCAGGCCTCTGAGGGCTCGAGGCGGTCAAGACACAGTG CTAGGATCATTGCCCAGACCACTGTTGATGCAAAACTCCATGCTGATTTTGAGGAGTC AGGCAGTTCCTTTGATTACTCAACGTCAGTGCGTGTTACTGGCCCGGTTGCGGAGAAC CAGCCACCGAGGTCTGACAAGGTGACCACGACTTATCTTCATCACATACAGAAGGGAA AGCTGATTCAGCCCTTCGGTTGTTTGCTCGCCTTGGATGAGAAGACCTTCAAAGTCAT TGCATACAGCGAGAATGCTCCTGAGCTGTTGACAATGACCAGTCATGCAGTTCCTAGT GTTGGCGAGCACCCTGTTCTAGGCATTGGGACGGATATAAGGAGCCTTTTCACTGCTC CTAGTGCCTCTGCATTGCAGAAAGCCCTTGGATTTGGAGATGTCTCCCTGTTGAATCC CATTCTTGTGCACTGCAAGACTTCTGCAAAGCCCTTTTATGCGATCGTACACAGGGTT ACAGGGAGCATCATTGTCGACTTTGAACCGGTGAAGCCTTATGAAGTTCCCATGACAG CTGCTGGTGCTTTGCAATCATACAAACTCGCTGCGAAAGCAATCACTAGGCTGCAATC TTTGCCCAGCGGGAGCATGGAAAGGCTTTGCGATACGATGGTTCAGGAGGTTTTTGAA CTGACGGGGTATGACAGGGTGATGGCTTATAAATTTCATGACGATGATCATGGTGAGG TTGTCTCTGAGGTTACAAAACCTGGACTGGAGCCTTATCTTGGGCTGCATTATCCAGC CACCGACATTCCTCAAGCAGCCCGTTTTCTGTTTATGAAGAACAAGGTTAGGATGATC GTCGATTGCAATGCAAAACATGTTAGGGTGCTTCAAGATGAAAAGCTTTCCTTTGATC TTACCTTGTGTGGCTCCACCCTTAGGGCACCACACAGCTGCCATTTGCAGTACATGGC CAACATGGATTCAATAGCATCTCTGGTGATGGCGGTTGTAGTAAACGAGGAAGATGGA GAAGGGGATGTCCCTGATGCTACTACACCTCAAAAGAGAAAGAGGCTATGGGGTTTGG TGGTTTGTCATAACACGAGTCCGAGGTTTGTTCCGTTTCCTCTCAGGTATGCCTGTGA GTTTCTAGCTCAAGTGTTTGCGATACACGTCAATAAGGAGGTAGAATTGGAGAATCAG ATTGTGGAGAAGAACATTTTGCGCACACAGACGCTCTTGTGCGATATGCTGATGCGTG ATGCTCCGCTGGGTATTGTGACACAGAGCCCCAACATAATGGACCTTGTGAAATGTGA TGGAGCAGCTCTCTTGTATAAAGACAAGGTATGGAAGCTGGGAGTAACTCCAAGTGAG 
Supplemental Table 2. Continued.

\begin{tabular}{ll}
\hline Gene ID & Coding sequence
\end{tabular}

TTCCATCTGCAAGAAATAGCTTCATGGTTGTGTGAATACCACACGGACTCAACGGGTC TGAGCACTGATAGCTTGCATGACGCTGGATTTCCTAGGGCTCTAGCTCTTGGGGATTC AGTATGTGGAATGGCGGCCGTGAGGATATCATCGACAGACATGATTTTCTGGTTCCGT TCTCATACCGCTGGTGAAGTGAGATGGGGAGGTGCAAAGCATGACCCAGATGATAGGG ATGATGCAAGGAGAATGCACCCGAGGTCATCTTTCAAGGCTTTCCTTGAAGTGGTCAA GACAAGGAGTTTACCTTGGAAGGACTATGAGATGGATGCCATACACTCCTTGCAGCTT ATTCTGAGAAATGCTTTCAAGGATGGTGAAGCTACTGATGTGAATACAAAGATCATCC ACTCGAAGCTTAATGATCTAAAAATTGATGGTATACAGGAGCTAGAAGCTGTGACCAG TGAGATGGTTCGTTTAATTGAGACTGCTACGGTGCCAATATTGGCGGTTGATTCTGAT GGATTGGTTAATGGTTGGAACACGAAAATTGCTGAGCTGACTGGTCTTCCGGTTGATG AAGCAATCGGGAAGCATCTCCTCACACTTGTTGAAGATTCTTCAGTGAAAATCGTTAA GAGGATGTTAGAGAATGCATTAGAAGGAACCGAGGAGCAGAACGTCCAATTTGAGATC AAGACGCATCTATCAAGAGCTGATGCTGGACCAATAAGTTTAGTTGTAAATGCATGCG CAAGTAGAGATCTCCATGAAAACGTGGTTGGGGTGTGTTTTGTAGCCCATGATCTTAC TGGACAGAAGACTGTGATGGACAAATTTACTCGGATTGAAGGTGATTACAAGGCGATC ATCCAGAATCCAAACCCGCTGATCCCGCCTATATTTGGTACCGACGAGTTCGGGTGGT GCACGGAGTGGAATCCGGCAATGTCAAAGTTAACCGGTTTAAAGCGAGAGGAAGTGAT GGACAAGATGCTGTTAGGAGAAGTCTTTGGTACGCAAAAGTCATGTTGTCGTCTAAAG AATCAAGAAGCATTTGTGAACCTTGGGATTGTGCTAAACAATGCTGTGACCAGTCAGG ATGCAGAGAAAGTGTCCTTTGCTTTCTTTACAAGAGGTGGAAAGTATGTGGAGTGTCT CTTGTGTGTGAGTAAGAAACTGGACAGGGAAGGTGTAGTGACCGGCGTCTTCTGTTTC CTGCAACTTGCCAGCCACGAGCTGCAGCAAGCACTCCATGTTCAGCGTCTAGCTGAGC GAACTGCATTGAAGAGATTGAAGGCTCTAGCTTACATAAAAAGACAGATCAGGAATCC GCTATCCGGGATCATGTTTACACGAAAAATGATGGAAGGTACTGAGTTAGGACCGGAG CAGAGACGGATTTTGCAAACTAGCGCTTTATGTCAGAAGCAACTCAGCAAGATCCTGG ATGATTCCGATCTCGAAAGCATCATTGAAGGGTGCTTGGATCTAGAAATGAAAGAATT CCGCTTAAACGAAGTGTTGACTGCTTCCACAAGTCAAGTAATGATGAAGAGTAACGGA AAGAGCGTTCGGATAACAAATGATACCGGAGAAGAAGTAATGTCTGATACTTTGTATG GAGACAGTATCAGGCTTCAACAAGTCTTGGCAGACTTCATGCTGATGTCTGTTAACTT TACACCATCCGGAGGCGAGCTAACTGTTACAGCTTCCTTGAGGAAAGATCAGCTCGGG CGATCTGTGCATCTTGCTTACTTAGAGATAAGAATAACGCATACCGGAGCTGGGTTAC CAGAGTTTTTGCTAAACCAAATGTTTGGAACTGAGGAAGATGTGTCGGAGGAAGGATT AAGCTTGATGGTTAGCCGGAAACTGGTGAAGCTGATGAATGGAGATGTTCAGTACCTG AGACAAGCTGGGAAATCGAGTTTCATTATCACTGCAGAACTCGCTGCAGCAAACAAG

TRINITY_DN68291_c2_g2 ATGGTTTCCGGAGGCAGTGGTGGCGACCGTGGAGGAGAAGCAGCGTCGTCAAGTCACC GCGTCGACCAGAACCTCCACACAACTCATAACCGGAGAGAACAAGCTCAGTCGTCAGG GACGAAATCTCTCAGGCCGCAAAGCCACACGGAATCAATGAGCAAAGCCATTCAGCAG TACACGGTCGACGCGAGGCTCCATGCCGTCTTCGAACAGTCCGGCGAGTCCGGCAAGT CGTTCGACTACTCGCAGTCTCTCAAAACCACGACCTACGGCTCCTCCGTTCCTGAGCA GCAGATCACGGCGTATCTCTCGAGGATCCAGCGTGGCGGCTACATACAGCCTTTCGGA TGTATGATCGCCGTCGACGAATCCACCTTCAGAATCATCGGATACAGCGAAAACGCGC GGGAAATGCTAGGGCTTATGCCTCAGTCCGTTCCTAGCCTCGAGAAACCGGAGATTCT AGCCATGGGAACGGATGTCCGCTCTCTCTTCACCGCCTCGAGCTCGGTTCTCCTCGAG CGTGCGTTCGTGGCTCGAGAAATCACGCTGCTGAATCCGGTTTGGATCCACTCCAAGA ACACTGGTAAGCCCTTCTACGCAATTCTACACAGGATCGACGTTGGTGTCGTTATCGA TTTAGAGCCAGCTAGAACAGAAGACCCGGCTCTTTCTATCGCCGGAGCTGTCCAGTCG CAGAAACTCGCCGTTCGCGCGATTTCGCAGTTACAGTCGCTTCCCGGTGGAGACATCA AGCTCCTCTGTGACACTGTTGTGGAAAGCGTCAGAGACTTAACTGGATACGACCGTGT AATGGTGTACAAGTTTCACGAAGACGAGCACGGAGAGGTTGTGGCTGAGAGTAGGAGA GACGATTTAGAACCTTACATCGGTCTACATTATCCGGCAACCGATATTCCGCAAGCGT CACGGTTCTTGTTTAAGCAGAACCGTGTCCGTATGATCGTAGATTGCCACGCCACGCC GGTTCTTGTGGTCCAAGACGATAGGCTCACTCAATCTATGTGTTTGGTTGGTTCTACT CTTAGGGCTCCTCACGGTTGTCACTCTCAGTATATGGCTAACATGGGATCTATTGCAT CTTTAGCAATGGCGGTTATAATTAACGGCAATGAAGAAGACGGGAGCAATGTCGGTGG TGGGAGAAGCTCTATGAGGCTTTGGGGTTTAGTCGTTTGCCACCACACCTCTTCTCGC TGCATACCGTTCCCTCTTAGGTATGCTTGTGAGTTTCTAATGCAGGCGTTTGGTTTAC AGTTAAACATGGAATTGCAGTTGGCTTTGCAAATGTCAGAGAAACGCGTTTTGAGAAC

Continued next page 
Supplemental Table 2. Continued.

\begin{tabular}{ll}
\hline Gene ID & Coding sequence
\end{tabular}

GCAGACACTGTTATGCGATATGCTTCTGCGTGACTCGCCTGCCGGAATTGTTACACAG AGTCCTAGTATCATGGACTTGGTGAAATGTGACGGTGCAGCGTTTCTTTACCACGGGA AGTATTACCCGTTGGGTGTTGCTCCGACTGAAGCTCAGATAAAAGACGTTGTGGAGTG GTTGCTTGCGAATCACGCGGATTCAACTGGGTTAAGCACAGATAGTTTAGGCGATGCT GGATATCCCGGTGCAGCTGCGTTAGGGGATGCTGTGTGCGGTATGGCGGTTGCGTATA TCACGAAAAGAGATTTCCTTTTCTGGTTCCGATCTCACACCGCTAAAGAAATCAAATG GGGAGGTGCTAAGCATCATCCCGAGGACAAAGATGATGGGCAACGGATGCATCCTCGT TCCTCTTTCAAGGCTTTTCTTGAAGTTGTTAAGAGCCGGAGTCAACCATGGGAAACTG CGGAAATGGATGCGATTCATTCGCTCCAGCTAATTCTAAGAGACTCTTTTAAAGAATC GGAGGCAGCTATGAACTCTAAAAATGCAGATGGTGCGGTTCAGCCTTATAGGGATATG ACAGGAGAGCAGGGAATCGATGAGTTAGGTGCAGTTGCAAGAGAGATGGTTAGGCTCA TCGAGACAGCAACTGTGCCGATATTCGCTGTGGACGCTGGAGGATGCATCAATGGATG GAACGCTAAGATTGCGGAATTGACTGGTCTCTCAGTTGAAGAAGCTATGGGGAAGTCT CTGGTTTCTGATCTAATATACAAAGAGAATGAAGAAACTGTCGATCAGCTCATTTCTC GTGCGCTGAGAGGAAACGAGGACAAGAATGTGGAGATAAAGCTGAAAACTTTCAGTCC CGAACTACAAGGGAAAGCGGTATTTTTGGTCGTGAATGCGTGTTCGAGCAAGGACTAC TCAAGCAACATTGTCGGCGTGTGTTTTGTGGGACAGGATGTTACTGGTCAGAAAATCG TAATGGACAAGTTCATCAACATACAAGGAGACTACAAAGCCATTGTTCATAGCCCAAA CCCGCTGATTCCACCAATCTTTGCGGCGGACGAGAACACTTGCTGCTTGGAGTGGAAC ACTGCGATGGAAAAGCTCACGAATTGGTCTCGCAGTGAAGTGATTGGGAAAATGCTTG TTGGGGAGGTTTTTGGTAGCTGTTGCAGGCTAAAGGGTCCTGATGCTTTAACCAAGTT CATGATTGTATTGCATAATGCGATCGGTGGCCAAGATACGGATAAGTTCCCTTTCCCG TTTTTTGACCGCAACGGGAAGTTTGTTCAGGCTCTATTGACTGCAAACAAGCGGGTAA GCCTCGACGGAAAGGTTATTGGGGCTTTCTGTTTCTTGCAGATCCCGAGTCCTGAGCT GCAGCAAGCTTTAGCAGTCCAACGAAGGCAGGACACAGAGTGTTTCACGAAGGCGAAA GAGCTAGCTTACATTTGCCAGGTGATAAAGAATCCTTTGAGCGGTTTGCGTTTCACAA ACTCATTGTTGGAAGCTACGGACTTGAACGAGGATCAGAAACAGTTTCTTGAAACGAG TGTTTCTTGCGAGAAACAGATCTCGAGGATTGTCAGTGACATGGATCTTGAAAGAATT GAAGACGGTACGTTTGAGCTAGATAGAGCGGAGTTTTTACTCGGAAGTGTCATAAACG CGATTGTAAGCCAGGCGATGTTCTTATTGAGGGAGAGAGGTGTTCAGCTGATCCGTGA CATTCCCGAAGAGATCAAATCGATAGCTGTTTATGGAGACCAGACAAGGATTCAACAG CTCCTAGCTGAGTTTCTGCTGAGTATAATCCGGTATGCACCGTCCCAAGAGTGGGTGG AGATCCATCTAAGCCAAGTTTCGAAGCAAATGGCTAATGGATTCTCCGCTATCCGCAC AGAATTTAGGTACATTTCATTGCACCCGCTTTTGTCTCCTCATCTCTGTAACCATCAT TACGCAATCTGGTTTACAGTTCCATATTTTTGGTTTATGAAGAAGGTTTTGATCGATT CCTTTTTAAAAAGGTTTGATTGTGTCTTGGTTGTTGCTTGTTTGGATATAGAATGGTT TGGTTTTTGGATCGTTATC

TRINITY_DN27276_c0_g1 ATGGTCTGCGGAGGCGGTGGCGAAACCAGCGGTAGAGGTCGCAACGGAGAGGCATCTT CGTTGAGCCACCGTGTGGATCAGAACAATCACCGCGGAAAACAGGCTCAGTCGTCGGC AAGCAAACCCCTAAGGTCACAGAATCAGCCGCGAAGCCACGCCGGCACGGAGTCCGTG AGCAAAGCTATCCAACAGTACACTGTCGACGCGAGGCTCCACGCCGTCTTCGAGCAGT CTGGAGAGTCCGGCAAGTCGTTCGACTACTCCCACTCCCTTAAAACGACGACGTGCGC TTCTTCCGTACCGGAGCAGCAGATCACGGCTTATCTCTCGCGGATCCAGCGCGGCGGC TACATCCAGCCTTTCGGTTGCTTGATCGCCGTCGACGAATCCACCTTCACCGTCATCG GACACAGCCAAAATGCGCGGGAAATGCTAGGGCTCATGTCCCAA

TRINITY_DN27276_c0_g2

ATGGTCTGCGGAGGCGGTGGCGAAACCAGCGGTAGAGGTCGCAACGGAGAGGCATCTT CGTTGAGCCACCGTGTGGATCAGAACAATCACCGCGGAAAACAGGCTCAGTCGTCGGC AAGCAAACCCCTAAGGTCACAGAATCAGCCGCGAAGCCACGCCGGCACGGAGTCCGTG AGCAAAGCTATCCAACAGTACACTGTCGACGCGAGGCTCCACGCCGTCTTCGAGCAGT CTGGAGAGTCCGGCAAGTCGTTCGACTACTCCCACTCCCTTAAAACGACGACGTGCGC TTCTTCCGTACCGGAGCAGCAGATCACGGCTTATCTCTCGCGGATCCAGCGCGGCGGC TACATCCAGCCTTTCGGTTGCTTGATCGCCGTCGACGAATCCACCTTCACCGTCATCG GACACAGCCAAAATGCGCGGGAAATGCTAGGGCTCATGTCCCAA

TRINITY_DN68291_c0_g1

ATGGATGCGATTCACTCGCTGCAGCTTATTCTAAGAGATTCTTTCAAAGAGTCCGAAG CGATGGACTGTAAAACCAATGCAGTTGGGGAGGTTCAGCCACATGGAGAGTATATGAC AGAGCAAGGGATGGAGGAGATAGGTGCAGTTGCGAGAGAGATGGTTAGGCTCATTGAG ACTGCGACGGTCCCTATATTTGCTGTGGACATTGACGGCTGCATCAATGGGTGGAACG 
Supplemental Table 2. Continued.

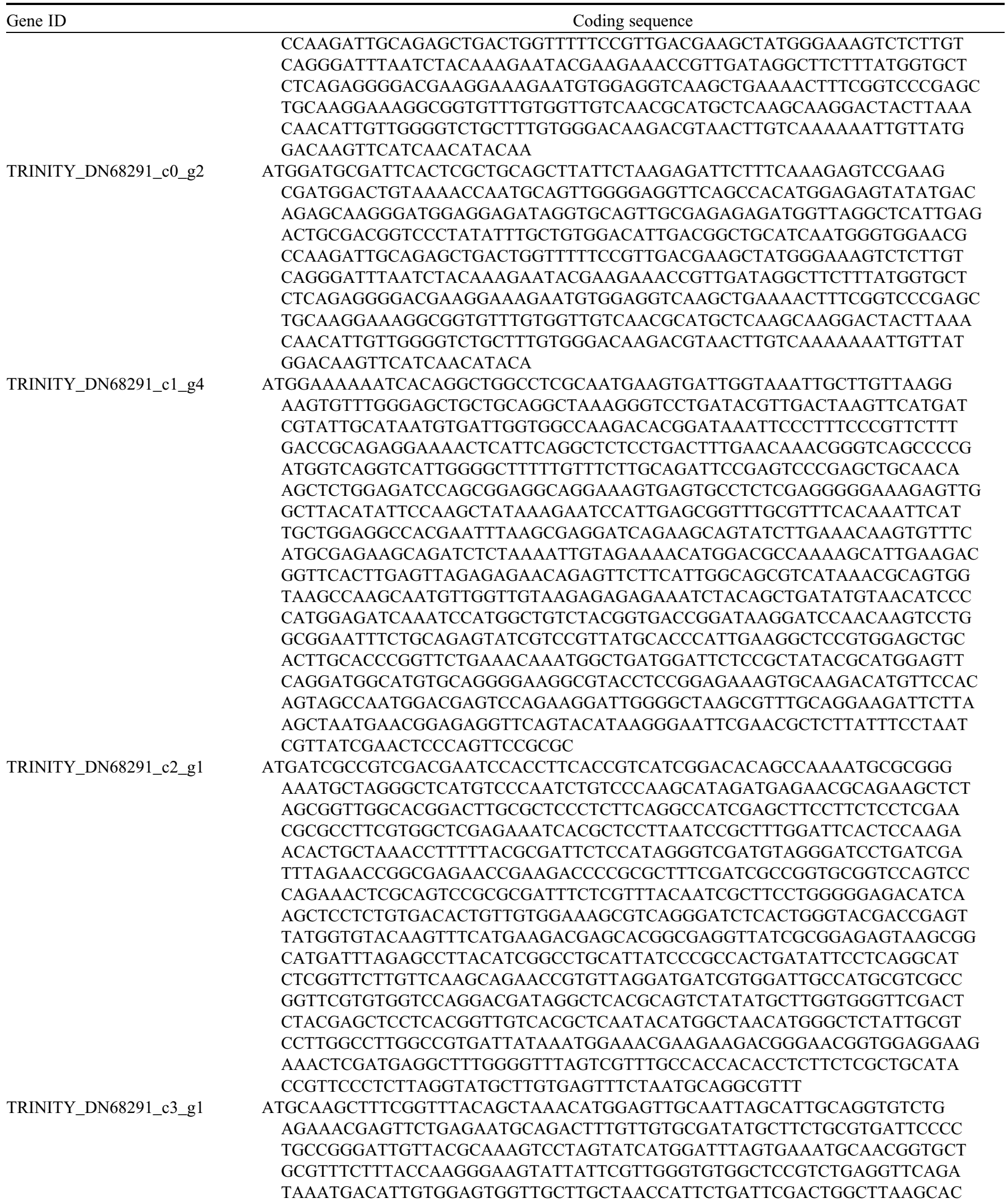

Continued next page 
Supplemental Table 2. Continued.

\begin{tabular}{ll}
\hline Gene ID & Coding sequence
\end{tabular}

CGATAGTTTAGGTGATGCGGGATATCCTCGTGCAGCTGCTTTAGGGGACGCTGTGTGC GGTATGGCGGTCGCGTATATCACGAAAAGGGACTTCCTTTTCTGGTTCCGGTCTCACA CCGAGAAAGAAATCAAATGGGGAGGAGCTAAGCACCATCCTGAGGACAAAGATG ATGGCCAGCGGATGCACCCGCGTTCTTCGTTCAAGGCTTTTCTTGAAGTTGTT

TRINITY_DN69481_c0_g1

ATGGGATTCGAGAGCTCTTCATCAGCTGCAAGCAACATGAAACCTCTTGGCGGCGATT CAAGACCACAAAATTCCAACACTGCTCAATACTCCGTCGATGCTGGCCTCTTCGCTGA CTTCGATCACTCCGTTTACTCCGGCAAGTCTTTTAACTACTCCAAATCCGTGATTTCG CCCCATAACAACGTTCCTGATGAACACATCACTGCTTATTTGTCTAACATCCAAAGGG GCGGTCTTGTTCAGCCCTTTGGTTGTTTGATTGCTGTGGAAGAGCCTAGTTTTAGGAT ACTCGGTGTTAGTGAGAACTGTATTGAGTTTCTTGGTCTGTCTCTTGCTTCGAGCTCT CATTCTAGCGAGTTTAAGGTTAAGGGTTTGATTGGAATCGATGCGAGGTCGCTTTTTA CTCCTTCTTCAGGAGCTTCTTTGGTTAAAGCTGCTTCCTTTACTGAGATTTCGTTGAT GAATCCGGTTTTGGTTCATTCTAGGACGACCCTGAAGCCGTTTTACGCTATACTTCAC AGGATCGATTCAGGGATTGTTATTGATTTGGAGCCTGCTAAATCAGCTGACCAGGCTT TGACTCTTGCCGGTGCAGTTCAGTCTCAGAAGCTTGCCGTTAGAGCCATTTCTAGGCT GCAGTCACTTCCCGGAGGAGATATTGGTGCTTTATGTGATACTGTTGTGGAAGATGTT CAGAGACTTACCGGTTACGACCGTGTTATGGTCTATCAGTTTCATGAAGATGATCATG GTGAAGTGATATCTGAGATTAGAAGATCTGATTTGGAGCCTTATTTGGGTTTGCATTA CCCTGCAACAGATATTCCTCAGGCTGCTCGGTTCTTGTTCAAACAGAACCGTGTCCGA ATAATTTGTGATTGCAATGCAACACCGGTTAAGGTTGTTCAGAGTGATGAACTCAAGA GACCACTTTGTTTAGTCAATTCTACACTAAGATCTCCTCATAGTTGCCACACGCAGTA TATGGCGAATATGGGCTCTATGGCTTCCCTAGTCCTCGCAATTGTGGTAAAAAGCAAA AACTCAACCAAGCTTTGGGGATTAGTAGTGGGTCATCATTGTTCTCCTAGGTACGTTC CGTTCCCATTACGGTATGCTTGTGAGTTTCTGATGCAAGCATTTGGACTTCAGCTTCA AATGGAGCTTCAGTTAGCGTCACAGTTAGCTGAAAAGAAAGCTATGCGGACACAGACT CTACTGTGCGATATGCTTCTCCGTGATACTGTTTCAGCTATTGTGACGCAGTCTCCTG GTATCATGGACCTTGTCAAGTGCGATGGAGCGGCTTTATACTACAAAGGGAAATGTTG GTTGATTGGTGTTACCCCTAACGAGTCGCAAGTTAAAGACTTGGTGAATTGGCTGGTG GAGAATCACGGTGAAGACTCGACGGGTTTAACCACTGATAGTTTGGTGGATGCGGGAT ACCCTGGTGCCATCTCACTTGGACATGCGGTTTGTGGTGTGGCTGCAGCGGGGATTTC TTCGAGAGATTACTTGATTTGGTTCAGGTCCAATACTGCAAGTGCCATCAAATGGGGA GGAGCTAAACATCATCCAAATGATAAAGATGACGATGGAAGAATGCATCCGAGGTCAT CGTTTAAAGCGTTTCTTGAAGTTGCCAAGAGCAGGAGCTTGCCGTGGGAAACCTCTGA GATTGATGCTATTCATTCCCTGAGGATTATAATGAGAGAGTCATTTACTAGCTCTAGG CCGGTATTGTCTGGTAATGGTGTGGCGAGAGATGCAAATGAGCTTACTTCTTTTGTGT GTGAAATGGTTAGGGTGATTGAAACCGCAACGGCGCCTATCTTTGGGGTTGATTCTTC TGGATGTATCAATGGTTGGAACAAAAAGATAGCTGAAATGACGGGGTTACAAGCTAGC GAAGCCATGGGGAAGTCACTTGCCGATGAGATTGTTCAAGAGGAATCACGCGCAGCTC TTGAAAGTGTCTTACGCAAAGCCCTACAAGGTAAAGAGGAGAAGAACGTAATGCTTAA ACTGAGAAAGTTTGGTCACAATGATCATCCTCCGGATTCTTCTTCTGATGTATGTGTT CTCGTTAACTCCTGCACGAGCCGGGATTACACAGAGAAAATCGTCGGTGTCTGCTTCG TTGGTCAAGACATCACTATCGAGAAAGCAATAACAGATAGATTCATCAGACTACAAGG AGACTACAGGACTATTGTTCAGAGCTTAAACCCGTTGATTCCACCGATATTTGCCTCG GATGAGAGTGCTCGTTGTTCAGAGTGGAACGCAGCCATGGAAAAGCTTACCGGGTGGT CAAAACACGAGGTGGTTGGGAAAATGCTTCCCGGTGAAGTCTTTGGAAATTTTTGTAA AGTGAAAAGTCAAGATTCACTCACAAAGTTCTTGATCTCTCTGTACCAAGGAATTGCT GGTGATAACGTTCCAGAGAGTTCACTGGTTGGTTTTTTTAACAAGGAAGGGAAGTACA TAGAAGCATCCTTAACCGCCAACAAGAGTACAAACACCGAAGGGAAAGTCATCGGATG TTTCTTCTTCTTGCAGATTATCAATAAAGAATCGAGTTCGAGCTGCCCAGAGCTGAAA GAGAGCGCTCAAAGCCTCAATGAATTAAGTTACATAAAGCAAGAAATCAAGAATCCTC TCAACGGTATCCGGTTTGCACATAAGCTTCTTGAATCCTCAGAGATTTCAGAGAGCCA AAGGCAGTTTCTCGAGACTAGCGATGCTTGCGAGAAGCAGATCACGACAATAATCGGA GTCACGGACTTAAAAAGCATCGAGGAAGGCAAGTTGCAATTGGAAACAGAGGAGTTTC GACTTGAAAGCGTCTTGGACACAATCATTAGCCAAGTGATGATTATGTTGAGAGAGAG GAAGTCACAACTCAGGGTTGAAGTCGCCGAGGAGATCAGAACTCTACCTCTCTACGGT GACAAAGTAAAGCTTCAGCTCATTCTTGCTGATCTTCTGCGCAACATTGTGAATCATG CCCCGTTTCCGGATAGTTGGGTAGGAATCAAGATCTCACCACACCACAAGCTTGCACG 
Supplemental Table 2. Continued.

\begin{tabular}{ll}
\hline Gene ID & Coding sequence
\end{tabular}

TGACGATGATGGCTATGTCCATCTACAGTTCAGGATGATACATCCTGGAAAAGGACTT CCTTCAGAGATGTTAAGTGATATGTTTGAGACTCGAGATGGATGGGTCACGCCTGATG GGTTAGGACTTAAGCTTTCAAGGAAACTGCTGGAGCAGATGAATGGTCGTGTGAGTTA TGTCCGAGAAGACGAACGTTGTTTCTTTCAAGTGGATCTTCAAGTCAAAACAAGGTTA GGTGTTGAGACCAGAGAAACAGAAGATGGTTCAAGCATAAAG

TRINITY_DN67850_c0_g2 ATGCCTATTGAAGAATTGCTTGCAAGGTATAAGGAGGATTTCGGTGACAAGGATTTGT CTGAAAATGACTCTGGTTATACATGTGCTCTATCTGAAGACTTTGTAGTAGAATCTCA CAAAACCAGGCAGCAAGCTGATTCAGATGACGAAAACGTTGATTTAGCAGAATGTAAG CCAGATTTGCAGCCTTGCACAGAGAAAGATGAAGGCACATCCAATGAACTGAAAGAAG ATAACGAGAAAGATAGTAGCGATAAAATTGCCGATGCCGCAGCAGCAGCAAGATCTGC GCAGCCAACTGGATTTACATACTCTACAACAAAAGTTCGAACGAAATTGCCGTTTCTT CTTAAGCATTCTTTGCGTGAGTACCAACACATTGGTTTGGACTGGCTTGTCACGATGT ATGAGAAAAGATTGAACGGGATTCTAGCTGATGAAATGGGTCTTGGAAAGACCATCAT GACCATCGCTCTTCTCGCACATCTTGCTTGTGATAAAGGGATATGGGGGCCCCATCTG ATTGTGGTTCCAACAAGTGTGATGCTTAATTGGGAAACTGAATTTCTTAAATGGTGTC CTGCTTTTAAAATTCTCACCTACTTTGGGAGTGCAAAGGAGCGGAAACTCAAGAGACA AGGATGGATGAAGCTGAACTCCTTTCATGTATGCATAACAACCTATAGGCTAGTTATC CAGGACAGTAAAATGTTCAAGCGCAAGAAGTGGAAGTACTTGATTCTTGACGAAGCCC ATTTAATTAAGAATTGGAAGTCACAGAGATGGCAAACTCTGTTGAACTTCAATTCTAA ACGCAGGATTTTGTTGACTGGTACACCGCTGCAGAACGATCTTATGGAATTGTGGTCA TTAATGCATTTTCTGATGCCACATGTCTTCCAGTCTCACCAGGAATTCAAAGATTGGT TCTGTAACCCGATAGCAGGGATGGTGGAGGGACAAGATAAGATCAACAGAGAAGTTAT TGATCGCTTGCATAATGTCCTCCGTCCTTTCCTTCTACGGCGCCTAAAAAGGGATGTG GAGAAACAACTTCCTTCGAAACATGAGCATGTTATTTTATGTAGATTATCTAAGAGGC AGCGCAACTTGTATGAGGACTTCATAGCAAGCACAGAAACACAGGCTACACTTAACAG TGGAAGCTTTTTTGGGATGATAAGTATCATAATGCAACTGCGGAAAGTTTGTAATCAT CCTGATCTCTTTGAGGGTCGGCCTATTGTTAGTTCTTTTGATATGGCTGGCATTGATG TGCAGTTGAGTTCGAGAATCTGCTCTCTGCTTATGAAAAGTCCATTTTCCAGGGTCGA TCTTGAGGCTCTGGGATTCTTATTCACCCATCATGATTTTTCTATGACTGGTTGGGAA GGGGATGAGATCAAAGCTATTTCAACGCCCTCAGATTTGATCAAGCAGCGCGTGGACT TGAAGGATAATTTAGAAGAAATCCCTCTGAGTTTAGTAAACCGTAAAAACCTGCAAGG GACAAATATTTTTGAAGAAATTCGAAAGGCCGTCTTTGAGGAAAGGGTTAAAGAATCT AAGGATCGGGCGGCAGCCATTGCATGGTGGAACTCTTTGAGATGCCAGAGAAAACCTA CTTACTCTACATCTCTAAGAAGTCTTTTAACCATTAAGGGTCCTCTTGATGACATACA CCATCTTAAGGCAAATCGTCCATCTTACATGTATTCCTCAATGCTGGCTGACATAGTT CTTTCACCAATCGAGCGTTTTCAGAAGGTAATTGAGCTAGTCGAAGCTTTTACGTTTG CGATTCCAGCTGCACGAGTGCCTTCACCTGCTTGCTGGTGCAGCAAAAGTGACTCTCC TGTTTTTCTTAGTCCATCGTACAAGGAGAAAGTTACAGATCTATTGTCACCTCTTCTG TCTCCGATTAGACCTGCTATTGTTAGGAGACAAGTATATTTTCCAGATAGGCGCCTGA TACAGTTTGACTGTGGTAAGCTGCAGGAGCTTGCCATGTTACTGAGAAAATTAAAATT TGGGGGTCACAGAGCGTTGATATTCACGCAAATGACCAAGATGCTTGACATCTTGGAG GCTTTCATTAGTTTATACGGTTATACTTACATGCGTCTTGACGGCTCTACTCCACCAG AAGAGAGGCAGACATTAATGCAGCGGTTTAACACAAATCCCAAGATTTTTCTTTTCAT TTTGTCAACTCGAAGTGGGGGTGTTGGCATAAACCTCGTTGGTGCAGACACGGTTATT TTCTATGACAGTGACTGGAATCCCGCAATGGATCAACAAGCACAAGATCGCTGCCACA GGATTGGTCAGACACGGGAAGTGCATATTTACCGGTTGATAAGTGAGAGCACCATTGA AGAGAATATCTTGAAGAAAGCAAACCAGAAGCGTGCGCTTGATAACCTAGTGATTCAG AATGGTGAATATAACACCGAGTTTTTCAAGAAGCTCGACCCCATGGAATTGTTCTCTG GGCATAAGGCTCTGACCACGAAGGATGAAGAGGTCAAAAGCAAGAATTGTGGGGCGGA GCTCCCTCTGTCAAATGCAGATGTTGAGGCTGCTTTAAAACATGCAGAAGATGAAGCG GATTATATGGCTCTCAAAAGAGTAGAAGAAGAAGAAGCTGTGGATAATCAAGAGTTTA CAGAAGAACCTGTAGAGAGACCAGAAGACGATGAGCTAGTAAATGAGGATGACATAAA GTCAGATGAGCCTGCTGATCAAGGTGTAGCTGCAGCAGGGTCGACGAAAGAAGAAATT CCATTGTTACCTACTGATAACAGGGATGAGAGAGCTGACATCACTATTTTAAGCCAAG AAGAAGATAATGATGTACCAGACGATGTCAAGCAAATGGCTGCAGCAGCCGCCGCTGC TGGACAAGCTATCTCATCTTTTGAGAATCAGCTGCGTCCTATTGATAGATATGCGATT AGATTTTTAGAGGTTTGGGACCCCATCATTGTTGAGGCTGCGTTGGAAAATGAAGCGG 
Supplemental Table 2. Continued.

\begin{tabular}{ll}
\hline Gene ID & Coding sequence
\end{tabular}

GATTTGAAGAGAAGGAATGGGAACTTGACCACATTGAGAAGTACAAGGAGGAAATGGA AGCTGAGATTGATGATGGCGAAGAGCCTCTTGTTTATGAGAAATGGGATGCTGATTTT GCAACCGAGGCATACAGGCAGCAAGTTGAAACTTTAGCTCAGCACCAGTTGATGGAAG ATTTGGAAAATGAAGCTAGAGAGAGGGAAGCTGCAGAGGAGGCTGAAATGTTTTTGAC ACAGAACGAAAGTGCTCATGTACTTAAACCTAAGAAGAAGAAAAAGGTGAAGAAAGCC AAATATAAATCCCTGAAAAAGGGATCACTAGCTGCCGAATCAAAACATGTGAAACCAG TGGTGGAAATTGAAGCTTCAATTGATGATGACAATGAAGAATGCGATTATGTAAGTAG CAGTGACTCAGACATGGTCTCACCGCATTTGCAGACAGGTATGAAAGGCAAGAAAAGA GAATTAATCTTCGACACTGAAGAAGAGAAGACCCCAAAGAAGAAAGCAAAGAAGCACA AGAAGTCTCTTTCACATTCAGATATCAAATGCAAGCAACAAAATACTCCACATGATGA ATTGGTTCTATCAAAGCCGTCTGACAGTATGGCTGTTGATAATGAATTGAAACCGGCA AACAGGGGTAAAACTATAGGGAAAAAATCCATCACTTCCATGCCAATAAAGCGTGTTC TTATGATCAAGCCAGAGAAATTGAAGAAAGGAAATCTTTGGTCTAGAGACTGTGTTCC CTCGCCTGATTCTTGGTTGCCTCAGGAAGATGCTATATTGTGCGCCATGGTTCACGAA TATGGGCCTAATTGGAGTTTTGTTAGCGGGACTCTTTATGGAATGACTGCTGGTGGGG CCTTCAGGGGAAGGTATCGCCATCCTGCATATTGTTGCGAGAGATATAGAGAGCTCAT CCAACGACATATTTTGTCTGCTTCCGACAGTGTAGTGAACGATAAGAACCTTAACACG GGTTCTGGGAAGGCTCTCCTAAAAGTCACTGAGGAAAATATGCGGGCACTCCTAAATG TTGCAGCCGAGCAGCCAGATACGGAGATGCTCCTTCAAAAACATTTTACTTGCTTGCT GTCATCAATTTGGAGGACGCCGACACGCACTGGGAACGATCAAATGCTCTCCTTAAAT AATCCTATATTCAATAGACAGTTTATGGGTTCTGCGAATCACACTCAAGAATTAGCTG GAAAACCTCTTCAGGGGATGAAGATCACCAGCTTAAGTAGCAAGTTGTTAGAATCCGC TCTTGAGGATTCAAGCACGAGTCAGCCACTGGATACTGTTTCCCGTTCTAGATTACAG GAGGATCAACCTATCAATAAAGTGGGATTAGACCTAACCCTGGAATTCCCACGTGGCA ATGATGATTCCCCCGTTGATTTTCCACCCATCATAAAGCTATCCATTGTTGGGTCAGA TTCATTCAATAACGTGAATGATCCTCCAGGGGAAGATAAGCTCAAAGCATCTCGGGTT GGTGCAGAGAACCGTTACAGGAATGCTGCAAATGCGTGTATTGAAGATTCATTTGGAT GGGCTTCAAACACGTTCTCCGCCTATGATCCCAAGTCGAGAACTGGAATGAAAGCACA GCCCCTGGGGAAGCACAAGCTATCAGCCTCCGACACACCCAAGTCCTCTAAAAGCAAA CAGCGAAAACTCTCAGCAGCAGAACAATCGGAAGTGGCATGGGTAAGGCCAAATGACC CGAATCTCAAGTTTGATTTTACACCTGCGGATACTGAGGAGGAAGAGGAGATGGCGGT TTCAGAAGAGATGGAGATGATAGGTTGTTCACATTGGTATGATCCTCTGTTAACCTCA GGCCTTGAGGATTGCTCATTAGCTTCCGAACTCTCAGAAATAGAG

TRINITY_DN46533_c0_g1

ATGCTTCGAGGATCTCGCTTTCTCTCTTCCCCGACTTCGATCTCTTCACTTTCCACCA CCCTCTTCTCTCAGGATCAACTCTCTGTGAAAGATTCTGTCTGTAAGCGCAACACCAT GCCTCTGTTTGAGCTATTCAGGCTCGCCAACGCTAAGCTTGAATCTGCTCAAGACAAT AACACTCCTCCACCTGTGGATGAAGTTGTCGAGCTGGTTTGGGAAAACGGTCAGATTT CTACTCAAAGTCAGTCGAGCAGATCGAGGAACATTCCTCCACCACAATCTGTTCATCA AGCAAGCTCTTCGAGACCTAGAGAGATTGGAAATGGCTCGAAGACGACGATGGTGGAC GAGATCCCTATGTCAGTGCCGTCTCTAATGACGGGTTTGAGTCAAGACGATGACTTGG TTCCATGGCTGAATCATCATCAATCCCTAGATGGATACTGCTCTGATTTCTTGCACGA CGTGTCTCCTGTTACTGTCAACGAGCAAGAGAGCGACATGGCGGTAAATCAAACTGTT TTCCCGTTGTTCCAGAGAAGAAACAATGGCAATGAATCAGCTCCTGCTGCTTCTTCAT CGCAGTATAACGGTTTCCAATCACATTCTCTGTATGGAAGTGATAGAGCTAGAGATCC TGTGAGCCAACCAGCCAAACCTGATCGATTTACTCAGCCACAGGAACCAGTAGTCACT ACTAGTAACAAGCCTGGTTTGATCAACTTTTCGCATTTCTTACGCCCTGCAACTTTGG CTAAGACTAATAACCCTCATTGCACTAAAGAGAAGAGTCCTCAAAGCCCGCCGAATGT GTTTAAGACCAAAGTTCTTGGAGCTAAAGACTCTGAAGTTAAGGTTCTTGACGAGTCT GCTCCTTCTGCAATGCCGAAGGATAACCAAAAGGCTTGCCTAATAACAGAAGACTCAG GTAGAAAAGAACAAGAGAGTGAAAAGGCCGTTGTATGTTCTTCTGTTGGCTCGGGTAA TAGTCTCGATGGCCCATCGGAAAGTCCTTCGCTTTCTTTAAAAAGAAAACATTCGGAT GTTCAGGACATGGACTGTCATAGCGAAGATGTTGAAGGAGAATCGGGAGATGGAAGAA AAGAAGTAGCTCCATCTCGAACGGGCATGGGTTCGAAGAGAAGCCGCTCGGCCGAAGT ACATAATCTGTCTGAAAGGAGACGACGTGATAGGATTAACGAGAAGATGCGTGCGCTT CAAGAACTAATACCAAACTGCAACAAGGTCGGCCTTTTTCTCCTTTGTGTATACTTGG TCACTCCTTTTCCGCCTTTCAAGGTATTGCTGCAACCAGTTTTTTCGTATGTGATCAG CTTCACTAACTTGAATGCTTTTTTTTATTGTTGTAAAAGGTGGACAAAGCTTCTATGC

Continued next page

J. Amer. Soc. Hort. ScI. 143(1):1-38. 2018. 
Supplemental Table 2. Continued.

\begin{tabular}{|c|c|}
\hline Gene ID & Coding sequence \\
\hline TRINITY_DN46533_c0_g2 & $\begin{array}{l}\text { ATGTCAATGGCGTCGGGTTACTACATGCCACCGGTTATGTTCCCACCGGGTATGGGGC } \\
\text { ATTACCAAGCAGCAGCAATGGCAATGGGTATGGGAATGCCTTATGCAATGGGCTTGCC } \\
\text { TGATATGAGCCGTGGCGGCCCCTCGGTTAACCAAGGACCACAGTTCCAGGTCCCGGGG } \\
\text { ATGCAACAACCAGTGGCAATGGCGATTCCACGTCTCTCTGGTGGTGGTTTCTTCACGG } \\
\text { GTTCTTCAACGACAGAGATGAATAAGAGTGACGATGGTTCGACGAGAGATATACCGGG } \\
\text { TACTAAAGGTCAGACGACGACAAAGAGCAACGATAGCTTGAAACCAATTAAAAGAAAA } \\
\text { CAAGCGTCTTCTGATCAGTTTTGTGGATCTTCG }\end{array}$ \\
\hline TRINITY_DN44852_c0_g2 & $\begin{array}{l}\text { ATGTTGGCTATGGACATCATCAGGCTCAGTCTCATGGATTATTCCAGTCTCTTGAATG } \\
\text { TGATCCCACTTTGCAAATTGGGTAAACCAAACAACTTCTTTGCATTTTTACACATCAA } \\
\text { CTGG }\end{array}$ \\
\hline TRINITY_DN51213_c0_g1 & $\begin{array}{l}\text { ATGTTTCCTACGCGCATCATCAAGCTCAGTCTCAAGGACTATATCAGCCTCTTGAATG } \\
\text { CAATCCAACTCTGCAGATCGGGTAAATCCTTTGTGATCTTTGCCTTGAAAAATCATCT } \\
\text { GCAAACCAGCTTGTGTACTTCACTACCAAGATTGTATTTGTTTGAGGCTCTT }\end{array}$ \\
\hline TRINITY_DN51213_c0_g2 & $\begin{array}{l}\text { ATGTTTCCTACGCGCATCATCAAGCTCAGTCTCAAGGACTATATCAGCCTCTTGAATG } \\
\text { CAATCCAACTCTGCAGATCGGGTAAATCCTTTGTGATCTTTGCCTTGAAAAATCATCT } \\
\text { GCAAACCAGCTTGTGTACTTCACTACTAAGATTG }\end{array}$ \\
\hline TRINITY_DN38410_c0_g3 & $\begin{array}{l}\text { ATGCAAGATACCATAGATCGTTATCTGAGGCATACCAAGGATCGAATCAGCAGCAAAC } \\
\text { CGGTTTCTGAAGAAAATATGCAGGTTAATTATGATATTGCGTACCAATTTCTTATAAC } \\
\text { AACTTTAGTACAGAAT }\end{array}$ \\
\hline TRINITY_DN69209_c0_g2 & 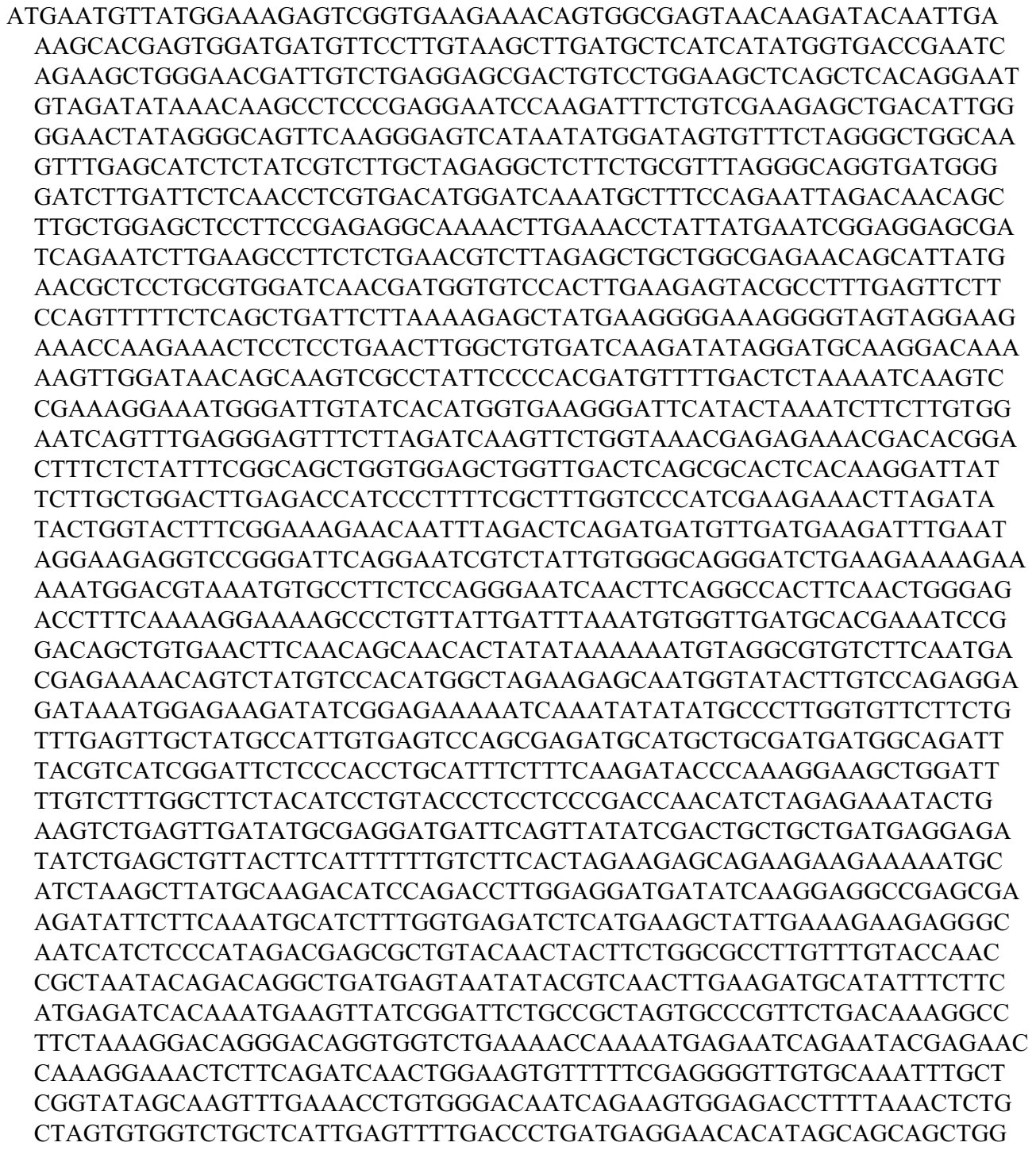 \\
\hline
\end{tabular}

Continued next page 
Supplemental Table 2. Continued.

\begin{tabular}{|c|c|}
\hline Gene ID & Coding sequence \\
\hline & GATATCAAAGAAAATCAAGATTTTTGACTTCAATGCGTTTATGAATGAATCTGTCGGT \\
\hline & GTTCATTATCCACTAGTGGAGATGGTCAACAAATCGAAATTAAGCTGTGTTTGCTGGA \\
\hline & ATAGTTACATCAAAAACTACTTGGCCTCGACTGACTACGATGGTGTAGTTCAGATATG \\
\hline & GGATGCCGGGACTGGACAAGGATTTTCGCAGTACACAGAACACCAGAAGAGAGCCTGG \\
\hline & TCAGTTGATTTTTCTCCATCTGACCCGACAAAATTTGTCAGTGGAAGCGATGACTGTT \\
\hline & CGGTAAAGCTTTGGAGCGTCAATGAGAAACGGTCACTAGGCACAATCTGGAGCCCAGC \\
\hline & AAATGTGTGTTGCGTGCAATTCTCTTCATATTCCAATCACTTATTGGCATTTGGGTCA \\
\hline & GCTGATTACAAGGTCTATTGTTATGATCTTCGGTATGTCAAAACTCCTTGGTGTACCT \\
\hline & TGGCGGGCCATGAGAAAGCTGTTAGCTATGTGAAATTTATGGATTCAGAGACCATTGT \\
\hline & TTCTGCTTCCACGGATAATTCTCTCAAGCTTTGGAATCTTAACAAGACGAATTCCTCT \\
\hline & GGTTTGTCTCCTGGTGCTTGTTCATTGACGTATAAAGGACATACAAATCAGAAGAATT \\
\hline & TTGTCGGATTATCGGTCTTGGATGGATACATAGCCTGTGGTTCAGAGACCAATGAGGT \\
\hline & ATATAGTTACTATAGATCCTTACCGATGCCAATGACTTCGTACAAGTTTGGATCAGTG \\
\hline & GATCCCATTTCTGGAAACGAATACTTTGATGACAATGGACAGTTTGTGTCGAGCGTCT \\
\hline & GTTGGAGAAAAAAGTCTAATATGCTTGTAGCTGCGAATTCGACTGGAAACATGAAGCT \\
\hline & ACTTAAACTGGTT \\
\hline \multirow[t]{5}{*}{ TRINITY_DN52784_c0_g2 } & ATGGAAGCTGTGAGTAGTATTGACGAGAGCTCGACAAGTACTGATTCCATTCACACTC \\
\hline & CGGCGAAGATTCCGCCGCCGACGACGAGAAAGTCGTCATCTCCGGCGAGTTTATACAG \\
\hline & AATGGGAAGCGGATCAAGCGTGGTTTTGGATCCCGAGAACGGCGTCGAAGCCGAATCA \\
\hline & AGAAAGCTCCCGTCGTCGAGATTCAAAGGCGTCGTCCCTCAGCCAAACGGAAGATGGG \\
\hline & GAGCTCAGATTTACGAGAAGCACCAGCGCGTGTGGCTCGGCACTTTCAACGAGGAA \\
\hline \multirow[t]{8}{*}{ TRINITY_DN33790_c0_g1 } & ATGCATGGAACAACCGATATTGTCCGGGAGCGGAGAAATCTAGTTACGACGGCCCGCG \\
\hline & GAAGTCAGTCAACGGCGTCTAGCGGCGGTTTCACGCTGGCAGTTGAAGAAAACAGCTG \\
\hline & CAACGGGGAGGCCGAGATCGTTCTCATGTGCAAACATTCTGGTGTTGAACTGGTCTCT \\
\hline & GTAAGACGGCACAGAAACCACACTTCCTCTTCGGTTCTGCTTGAAAAGCAAATACACA \\
\hline & AATCGGTGGATCCCTATGTTTGGCCGAGGCATCTCGTACCCTACAATCTCCTTTCCAA \\
\hline & ATGTCACGTCGGTTGTCCCTGGGATATCGGTAACAATCCAGTGCAAGTGCTCTCTCAG \\
\hline & ATAAGGATCACTAGGTCCAGGAACATCAGGATCAGTCATGACCTTAGGTTTGTTTGTA \\
\hline & ACTGCAGAAGGGAAAAGTTCATGGCCGTTGTAGAC \\
\hline \multirow[t]{10}{*}{ TRINITY_DN21486_c0_g1 } & ATGGAGAATATGGGAAGTAGAGTGATAGAGCCATTGATAGTGGGAAGAGTGGTCGGAG \\
\hline & ATGTTCTCGATTTCTTCACTCCAACAATTAAAATGAATGTGAGTTACAACAAGAAACA \\
\hline & AGTCTCCAATGGCCATGAGCTTTTCCCTTCGTCTGTCTCCTCCAAGCCTAGAGTTGAG \\
\hline & ATTCATGGTGGTGATCTCAGATCCTTCTTCACCCTGGTGATGATAGACCCTGATGTTC \\
\hline & CAGGTCCTAGTGACCCCTTTCTAAAAGAACACCTGCATTGGGTCGTCACAAACATCCC \\
\hline & CGGTACAACAGATGCTACATTTGGAAAAGAGGTGGTAAGCTATGAGTTGCCAAGGCCA \\
\hline & AGCATAGGGATACACAGGTTCGTGTTTGTTCTGTTCAAGCAGAAGCAAAGACGTGTTA \\
\hline & TCTTCCCAAATATTCCTTCCAGAGATCACTTCGACACTCGCAAATTTGCGATCGAGTA \\
\hline & TGATCTTGGTCTCCCTGTCGCGGCTGTTTTCTTTAACGCTCAGAGAGAAACCGCAGCT \\
\hline & CGCAGACGT \\
\hline \multirow[t]{11}{*}{ TRINITY_DN61367_c0_g1 } & ATGGTCCGACAAGTTAGTTTGGTTGAAGAGGAGAAAGAATACGATCCGACACTCAGCG \\
\hline & AGCTAAGGGGACCAGTCATCAACAGTAACGGTGCGGGGTGTTCCCAAGGAGGAGGCAT \\
\hline & TGGTTCAGAAGGTGATAATGTAAGACCGAATGGCCTTCTCAAGGTATATTCTAAGGAG \\
\hline & CTTGATAAGAACAGTCGTTTCATGGGTGCTAAGCGGAGAAAGTCTGGTTCTGTGAAAA \\
\hline & GATTCAAACAAGACGCATCCACGAGTAACAACCACACAACAACTGCAGATCAGAATGC \\
\hline & AACACCAGAGTTGGCCACATTAGACTCTTTTGGTGGAATCGCGAGGATAGGGAATGAG \\
\hline & TATCCTGGTGTGCTGGAAAATAATAATTTATCTCAGAAAAGCAAAATGGAAGAACTGG \\
\hline & ACATCGCGAGGATCCTTAAACCAGTGAAATTTTCTTCATCTGTAACAAACAATGTACA \\
\hline & AGACGTGTTGGTGACCTTTTTGGCGCTAAGGTCTGATGGGAAAGAAGTGATGGTAGAC \\
\hline & AACAGATTTCTCAAGGCTCACAATCCTCTTCTGCTGATTGAATTCTACGAGCAACATC \\
\hline & TCAAGTACAATCCCGAAAGG \\
\hline \multirow[t]{7}{*}{ TRINITY_DN70446_c2_g2 } & ATGGATTTGAACGGCGAGTGTAAGGGAGGAGATGGGTTCATTGACAGAAGCAGAGTCA \\
\hline & GGATTTTGCTTTGTGACAATGATTCAAAGAGCTTGGGAGAGGTTTTCACCCTCCTTTC \\
\hline & ACAGTGTTCTTATCAGGTGACATCAGTGAAATCAGCAAGGCAAGTGATTGATGCACTA \\
\hline & AATGCAGAGGGACCTGATATCGATATAATTCTGGCGGAAATTGATCTCCCAATGGCCA \\
\hline & AGGGTATGAAGATGCTGAGGTACATCACAAGAGACAAAGATCTTCGGAGAATCCCTGT \\
\hline & GATAATGATGTCGAGGCAAGACGAGGTACCTGTTGTCGTAAAGTGCTTGAAGCTTGGT \\
\hline & GCAGCTGACTACCTTGTGAAGCCTCTTCGGACCAACGAGCTTCTGAACTTGTGGACAC \\
\hline
\end{tabular}

Continued next page

J. Amer. Soc. Hort. SCI. 143(1):1-38. 2018. 
Supplemental Table 2. Continued.

\begin{tabular}{|c|c|}
\hline \multirow[t]{24}{*}{ Gene ID } & Coding sequence \\
\hline & ACATGTGGAGAAGAAGACGCATGCTAGGACTTGCTGAGAAGAATATGTTTAGCTATGA \\
\hline & TTTTGATCTCGTGGGATCTGATCCAAGTGATCCAAACACAAACAGTACCACCCTGTTC \\
\hline & TCTGATGATACAGATGACAGAAGTATTAGGTCCACCAACCCGCAGAGAGGAACTTTAA \\
\hline & GCCATCAGGAAAAGGAGTGGCCTGTTGCTACGGGTTCCGTTTGTGCTGGCGATGGTGC \\
\hline & TGATGGCACAGCCACTTCAAGTCCTGCTGTTGCTATTATAGAGCCTCCATTGAATCAT \\
\hline & CTTCCTGGGTCTCACCATGAGCCTACGAAAAGAAATACCAATCCAGCGCAATTTTCTT \\
\hline & CAGTGCCAAAGAAAAGTAGATTGAAGATTGGAGAGTCCTCCGCGTTCTTCACATATGT \\
\hline & CAAGTCTACTGTCCTTGGTACCAACTGTCAGGATCCTCCCCGTGTCAATGGAAATGGC \\
\hline & TCACTTCATCTTCATCCAGGTATGGCGGAGAAGTTTCAAGCGGTGGCCAGTGAGGTGA \\
\hline & TCAACAACACTAAACAAACACGCCGAGGTAGAGAGGCCGAGAAAACCTGTTCTCAAGG \\
\hline & AGAGAACTTACAGAATGGCGCCAGCTATCCAAATTCCCTTGAGCGGTCTCGCACGCTT \\
\hline & CCAACACCAATGGAACTTCATGGTAGGAGTTACCAAGAAGGAAATATGAATACTCCCC \\
\hline & GGGATGCTATAAGCAGAAGTAAGGATTCATCTCAAGTTGATGCTTCGAGTTTCGCTGC \\
\hline & ACAGAATGCCTATCCTTACTATATGCATGGTGTCATGAACCAAGTTATGATGCAATCA \\
\hline & GCAGCCATGATGCCTCAGTATGGTCATCAACATCCTCATTGCCCACCTAATCATCTGA \\
\hline & ATGGAATCACAGGATTTCCTTATTACCACCACCCCATGAACACATCGTTACAGCACAG \\
\hline & CCAGATGCCACAGAATGGTCAGGTGCCTTTACAGAGTGGTCAGATGTCTTTACAAAAT \\
\hline & GGTCAGATGTCTATGGTTCATCATCATTCGTGGCCACCGGTGGGAAATCCGTCTTCTA \\
\hline & ACGAGGTGAGAGTGACTAAACTTGACAGAAGAGAGGAAGCTTTGCTCAAATTTAGACG \\
\hline & TAAAAGAAACCAAAGGTGTTTTGATAAGAAGATTAGGTATGTGAATAGGAAGCGCCTT \\
\hline & GCTGAGAGGAGACCGCGTGTTAAGGGTCAGTTTGTTAGGAAAATGAACGGCGTAAATG \\
\hline & TTGACTTAAACGGGCAGCCTGACTCTGCTGACTATGATGACGAGGAAGAGGAGGAAGA \\
\hline & AGAAGAAGAAGAGGAGAATCGGGACTCATCTCCTCAGGATGATGCTCTGGGAACT \\
\hline \multirow[t]{3}{*}{ TRINITY_DN34145_c0_g1 } & ATGCGATTGATCGTGTATTTGTTTTGGCAGCTAGGACTTGCTGAGAAGAATATGTTTA \\
\hline & GCTATGATTTTGATCTCGTGGGATCTGATCCAAGTGATCCAAACACAAACAGTACCAC \\
\hline & CCTGTCCTCTGTA \\
\hline \multirow[t]{3}{*}{ TRINITY_DN55625_c0_g1 } & ATGCATCTAAAAACTATGAATATGGTAGGAAAATCAACAAAAGAGGTTACTTCAAAAT \\
\hline & GTCTATTTTTTTTTATCACATCTGATCAAGGAACCAGGGCAAGGAACAACAACTCATT \\
\hline & TAAAAGCTTGCGTAGCACAAGAACATGGCGAAGGCCACAAGAACCC \\
\hline \multirow[t]{4}{*}{ TRINITY_DN55625_c0_g2 } & ATGTTCTCGTGCTACGCAAGCTTTTTAATGAGTTGTTGTTCCTTGATCAGATGTGATA \\
\hline & AAAAAAAAAGACATTTTGAAGTAAACTCTTTTGTTGATTTTCCTACCATATTCATAGT \\
\hline & TTTTAGATGCATTTATAATGTCTGTTGCCTTCTTTTGAACTTTTTGACAATGAATATT \\
\hline & GTGGATGTGTGCTTTTTATTGGAATGCAGTGACATCAGTGAAATCAGCAAGGCAAGTGAT \\
\hline \multirow[t]{23}{*}{ TRINITY_DN64509_c0_g1 } & ATGGATTCGTCTCTCGATGGAGCTGCAGGTGACTCATCGAAGTGCAGTGAAATGAGTG \\
\hline & TTGATGAGAAGAGACAGCTTGTTTATGAACTGTCGAAGCAATCACATCTAGCTTCTGA \\
\hline & AGTGTTGCAGGCATGGAGCCGCCAAGAGATTCTACAGATCTTATGCGCAGAAATGGGA \\
\hline & AAAGAAAGGAAATATACTGGCTTGACCAAAGTCAAGATCATAGAGACTCTATTGAAAA \\
\hline & TTGTATCTGAGAAAAAATCTGGAGAGTATGATGGGAAGAAGAGAGATTCTGACTGTGT \\
\hline & GCCTGTTCAGAGAAACTCAAAGAGACAAAGGAAGGTCGATAATCCTACTCGTTATGTG \\
\hline & ATTCCAACGAGCAACAATGCCTCAGGAAGCTGCAACTCGGTTAATACTAAAGGTGAGG \\
\hline & AGAATACTATCTACTGCAACAATTTGGCTTGCCGGGCCATATTGAGACAGGAAGACTC \\
\hline & GTTTTGCCGAAGGTGTTCTTGTTGCATTTGTCGTAAGTATGATGATAACAAAGATCCT \\
\hline & AGTCTCTGGTTGACATGTAGCTCAGATCCTCCATTTGAAGGTGATTCTTGCGGATTCT \\
\hline & CGTGTCATCTTGAGTGTGCTTTCAAGAGTGAAAAATCTGGTCTTGCAAAAGACAAGCA \\
\hline & AAGTGAGGGTTGCTGCTTCTACTGTGTCTCTTGCGGTAAACCAAATAGCTTGCTTGAA \\
\hline & TGTTGGAAGAAGCAACTGACGATTGCTAAAGAAACCAGAAGGGTAGACATACTATGCT \\
\hline & ACCGTCTTCTATTGGTCCAGAAACTAATAAAAGGCTCTACTAGATACCCAAATCTGTG \\
\hline & TGAATTTGTGGATGAAGCTGTGAAGAGTCTTGAAGCTGATGTGGGTCCTCTCACTGGT \\
\hline & СTTCCTATGAAAATGGGTAGAGGAATTGTAAACAGACTACAATCAGGACCCGATGTGC \\
\hline & AGAAACTTTGTTCTTCTGCTCTTGAAACACTAGAAACTACACCTCCCGAAGTCGCTGC \\
\hline & ATTGCCTTCACCTCGCAGCTACAAGATGCAGCACGATTGTTCTTACGGATTGAGCAAT \\
\hline & GAGATATCTGCAGATACAGCAACCACAGTATCAACCAAGATCAGATTCGAGGATGTTA \\
\hline & ACGCGACTTCGCTCACAGTGATTCTCGCCTCTAACGAAGTCGCCTCACCTGCAAACAT \\
\hline & CGTTCACTACAGTATCTGGCATCGTAAGGTCACCGAGAAAGATTACCAGGAGAAGTCG \\
\hline & ACTTGCACATTGTTTACTCCAAACGCAAGATTTGTTGTCTCTGGATTAGCTCCAGCGT \\
\hline & CTGAGTATTGCTTCAAAGTTGTGTCCTTTAGCGGTACACGAGAACTAGGCGTCGATGA \\
\hline
\end{tabular}

Continued next page 
Supplemental Table 2. Continued.

\begin{tabular}{ll}
\hline Gene ID & Coding sequence
\end{tabular}

AATCAATGTTTTTACGCGTAGCCCTGAAGAAGGAGCGAATTGCTCTTCGGTAGTCGAA AGAAGCGAAAGCCCTCTCACAAATTGCAGCACGTTGTCTTCGAATCCTTCTTCTGTTG AAGCTGAATCCAACAACGGTTACATTGCTCCTCAGAATCCCTCGCAGAACGACAAAAA AGATTCTCCTTCGACTGAGGAAAATGCTGCGAAAAGGACAACCGATTCAGACATTGTG CAGACTGAGAAAAACGTTGAAGTGTTGTTGGATGAAGAAGCTGTTCCCGACAAGAACG GAGGAACTCTAGTCCCGGTTAGTACGACAAATCTTGTTAGTAATCGAAACAGTTCAGA TGCGAGTTTGCCAATTACTCCCTTCAGGTCAGACCAAACCAAGAACCGTCAAGCTAGA AACGGAAAATCGGTTAAAGAAAACGGTAATAACGGTGATCATCACTCAGCGAACGGAG GATCAGAGAGCGGATTAGAGCATTGTGTGAAGATCATAAGACAGCTTGAATGTTCAGG ACATATCGAGAAGGATTTCAGACAGAAGTTTCTGACTTGGTATAGCTTAAGAGCGACG CCACAAGAGATTAGAGTTGTGAAGATATTCATCGATACGTTTACTGATGATCCCATGG CTTTAGCTGAGCAGCTCGTTCATACTTTCAACGACCGCGTTTCGATTAAGCGTTCTGC GATTGGCAATGGTGCGTCTGCGGTTGTCCATTCTGGATTCTGCATGAAGTTGTGGCAT

TRINITY_DN53167_c0_g1

ATGCCACGCCCTTTCTTCCACAAGTTGATTTTCTCATCCACTATCCAAGAAAAACGTC TGAGAGTTCCAGATAAGTTTGTGAGTAAATTCAAGGATGAGCTTTCGGTTGCGGTTGC ACTCACAGTACCTGATGGTCATGTTTGGCGTGTAGGACTAAGGAAAGCTGACAACAAA ATCTGGTTTCAAGATGGTTGGCAAGAGTTTGTTGACCGTTACTCCATTCGCATTGGTT ATCTTTTGATTTTTAGATACGAAGGGAACTCTGCCTTCAGCGTCTACATTTTCAATCT ATCACACTCGGAGATCAACTACCATTCCACCGGTCTCATGGATTCCGCACACAACCAC TTCAAACGCGCCCGTTTGTTTGAAGACCTTGAAGACGAAGACGCCGAGGTCGTTTATC CTTCTTCCGTGTATCCTTCACCGCATCCTGAAACTAATGTACCCGCCAATAAAGGGTA TACGAGTTCAGCCATCCAAAGCTTGTTCACTGGACCTGTTAAAGCTGAAGAGACAACA CCAACCCCAAAAGTTCCGAAAAAAAGAGGGAGGAAGAAGAAAAACGCTGATCCTGAGG AAATAAACTCATCTGCTCCGCGCGATGATGACCCAGAGAGCCGTTCAAAGTTCTACGA GAGCGCTTCTGCGAGAAAGAGAACCGTGACCGCAGAAGAGAGAGAGAGGGCCATCAAT GCAGCCAAAACGTTCGAGCCAACAAACCCTTTCTTCAGAGTTGTTCTGCGACCATCCT ATCTATACAGAGGTTGCATCATGTATCTGCCTTCTGGGTTTGCTGAGAAGTATTTAAG TGGGATCTCCGGATTCATCAAGGTCCAGCTCGCGGAGAAACAATGGCCCGTGAGATGC CTCTACAACGCAGGGAGGGCCAAATTCAGCCAAGGGTGGGACGAGTTCACGCGGGAGA ATAACCGAGAGAGAGGGCCATCAATGCAG

TRINITY_DN52604_c0_g1 ATGTATCTGCCTTCTGGGTTTGCTGAGAAGTATTTAAGTGGGATCTCCGGATTCATCA AGGTCCAGCTCGCGGAGAAACAATGGCCCGTGAGATGCCTCTACAAAGCAGGGAGAGC CAAATTCAGCCAAGGATGGTACGAGTTCACTCTGGAGAATAACCTAGGAGAAGGTGAC GTCTGCGTGTTCGAGCTGCTCAGAACCAGAGATTTCGTTCTGAAGGTGACGGCCTTTC GAGTCAACGAGTACGTC

TRINITY_DN21929_c0_g1

ATGTGTAGGCATAATAGTCGAGCGAAAGCCTCGCAGGAGGAGGTGGCTTCACCTGATG AGGATCTCTTGGTATTTTCTAAACCTGTTCGACTATACAACATTCTTCTCCGTCGCTC TCGATACAACCCATCGTTCCTTCGAAGATGCTTGAAGTACAAAATCCAAGCAAAGGGC AAAACAAAGTCCGAATCTGGTGGGAAGGTAGTTTTCAACTACAAGGATTGTAATAACA CATTGCAGAAAACTGAAGTTAGGGAGAATTGTTCTTGTCCATTTTGCTATATGCTATG TGGTAACTTCGAGGGGCTGCAACTTCATTTGAACTCATTTCATGGCTTATTTGAATTC GAGTGCATGCGTTCAGGAGAAGAGCAGAGAGTTAATGTCTCTGTAAGACTTGATAGAT TCGAATCTGAGGACGAAGAAACTCAATATGAAAAATTTGAGGAGCACTCTTTCTGCTC GAAACCTCGTAAGCGTAAACAAAGAGGTGGTGGTGGTGGAAATATGGCCAAGCGACTT AAAATAAGCATTTTACCATTGGATTCACCCAGTTTAGCCAATGCCACAGAAAATGGAA TCGCCCTGCTGAATAGTGATCTTGCTAGACAAGTTGGGATGAATTCACCAGCCGTAGC TCAATCTTCTCTGGACTCTGATGCTAATGCTATGTTACTAAGCGAAGCTGTGGTTGCT GCTGCCAAGACGAGAAAGTTATCTGCTGAGCGATCAGAAGCTAGAAGCAACCAACTTC TCCTGAAACGCCAGTTCTATCATTCTCACAAAGTTCAGCCAATGTCAATTGAGCAAGT AATGTCTGATCGAGATAGCGAAGATGAAGTTGATGATGACGTTGCAGAATTCGAAGAT CGCCAGCTGCAGATGCTTGATGATTTTGTAGACGTAGACAAAGAGGAAAAGAGATTCA TGCATCTTTGGAACACATTTGTAAGAAAGCAAAGGGTTGTGGCGGATGGTCATGTTCC TTGGGCGTGTGAAGCATTTTCAAAGTTTCACAAGAAAGACTTGAACCATTCCCTACGA CTCTCCTGGTGCTGGAGATACCATCTGATTAAACTCTGGGACCTTGGGCTTATCGACG CGGTCACCATGAACAACTGCTCTCTCATTCACCTGGAGAAATGCGATGACAACTCAGA CAACGCGAGTGTGGATCATCAGTCAGACAACAACAACAATGTCGATCATGGCATTGAC TTAAACACTAACGATAGGATGGATGTTGAGGAGGATGTCAATGACAGAAAAGACAAG 
Supplemental Table 2. Continued.

\begin{tabular}{|c|c|}
\hline Gene ID & Coding sequence \\
\hline TRINITY_DN33663_c0_g1 & $\begin{array}{l}\text { ATGATGGTGGCTTATTCTTGCGTCTTAGGATCACCGACAAAGAAGGTAACAGCTATCA } \\
\text { CTTTTACTATTGCTCTTATCCGTAATTTAATTCATAACCTA }\end{array}$ \\
\hline TRINITY_DN54268_c0_g1 & $\begin{array}{l}\text { ATGAACAATCTGAGTTATCTTGAACCAGATTACTCTGAGTTTGTTGAAGTTGATCCTA } \\
\text { CCGGAAGATATGGAAGATATAATGAAGTTCTGGGTAAAGGAGCTTCAAAGACTGTTTA } \\
\text { CAGAGCATTTGATGAATATGAAGGGATCGAAGTAGCATGGAACCAAGTGAAGCTCTAC } \\
\text { GATTTCTACAGAGCCCTAGGATCTCGAGAGGCTTTACTGTGAGATTATCTCCTA } \\
\text { AGACCCTAAAACACAAGAACATCATGAAATTCTACACCCTTGGGTTGATACCGCCAA } \\
\text { TCGAAACATCAATTTGTCACTGAAATGTTCACTTCTGGCACCTTAAGACAGTATAGA } \\
\text { CTCAGGCATAAGAGAGTGAACATAAGAGCGGTGAAACATTGGTGTAGACAAATCTTGA } \\
\text { GAGGCTTGCATTATCTTCACAGCCATGACCCTCTGTAATCCACAGGGATCTCAAATG } \\
\text { TGACAACATTTCGTTAATGGGAATCAAGGAGAGGTCAAGATTGGAGATCTTGGCCTC } \\
\text { GCTGCCATTTTAAGAAAGTCCCACGCTGCTCACTGCGTCGGGACGCCTGAGTTCATGG } \\
\text { CTCCTGAAGTTTACGAAGAAGCGTACAACGAACTGGTTGATATATACTCGTTCGGTAT } \\
\text { GTGCATTTTGGAAATGGTTACGTTTGATTACCCGTACAGCGAATGTACTCACCCTGC } \\
\text { CAGATCTACAAGAAAGTCATGTCGGGCAAGAAACCGGATGCGTTGTACAAGGTGAAAG } \\
\text { ACCCCGAGGTTAAATATTTCATCGAGAAATGCTTGGCCACCGTATCGCTGAGAGTCTC } \\
\text { AGCTCGTGAGTTACTAGATGACCCTTTTCTTCGAATAGACGATGGTGAATTTGATTTG } \\
\text { AGATCAGTTGATTTGGATGATTCGGTGGGGCCACTAGTTAGGCAGCCGCATCATCTTG } \\
\text { CTGACTACTACAACTACCCGTCGAACAGTAGCTCTTTGAATCGTCAGTACTCAAATGG } \\
\text { TTATAACAGCCACCACGAGTATCAAAACAGATGGGCGTATAATCCAGCTGAGACAGAG } \\
\text { GAGACTCACGGAATCGAGCTCTTTGAGTGTCGAAACGATAATGATCGTGAAGAAGACA } \\
\text { AGAGCTCTGGTAATGTTGATATAAGCATCAAAGGCAAGAGAAGAGATGATGGTGGCTT } \\
\text { ATTCTTGCGTCTTAGGATCACCGACAAAGAAGGGCGTGTCCGAAACATTTACTTCCCA } \\
\text { TTTGACATCGAGACAGACACGGCATTGAGCGTCGCGACAGAGATGGTAGCGGAACTTG } \\
\text { ATATGGACGATCACGGAGTCACAAAAATCGCCAACATGATTGACGGTGAGATATCTAC } \\
\text { TCTTGTGCCTAGTTGGAGACCGGGACCTGAATTCGAAGAATCTCTTGCGGCCGCGGCG } \\
\text { GCAGCAAATGCGAACATTTGCAGCAACTGCGTATCGAACCGCACCTCAATGGGATCGG } \\
\text { TGATGGATTTCCTGAGGACCAACCCTGGTGCAAATGTTGCACAGTGTTGCAGAAACGG } \\
\text { GTGCGGCGAGACTCATGGCCGGTTTGAAGAGATCACGATAAGAGAAACCGAGGTTCGT } \\
\text { CTTAGAGAGATATGGAAGCTGCAGCAACAGCAGGAAAGTCGTGAGCTTAGCTCGATAG } \\
\text { ACTCGGGGCAAAACCACTCCGAAGAAGAAGAGGAAGAAGAAGTATACGAGAACCCCGA } \\
\text { AATCACGTTTCTTGTGAGGCAAGTAACGCGCTAAACCATCTATCCGGTTCTGGATCG } \\
\text { TTCTCGTTTTGCCGTCTTTATACTGTGACGAGCTAGCCGAGAAAACCGAAAGTCAGG } \\
\text { TCCAACAAGAGTTGAGATGGCTTAAAGCTAAATGCCAAATGGAGGTCAGAGAGATGCA } \\
\text { GGATGAACAACTAAAATCCCGGTGGCCGGAATCAGGAGAAGAAGAAGCGGAAATATGT } \\
\text { CTGCAAGACGGGTTCTCGGGTTCGGTTTCGGGCTTGGGAAGTGGAGATGAAGATAAGG } \\
\text { TGAAAGAGAAGATGTTTGGAGGAAGATTAGTACCAAAATGTCTTAAAAGGACAACTTC } \\
\text { ACTTCCTGTTGATGCCATTGAATCT }\end{array}$ \\
\hline
\end{tabular}


Supplemental Table 3. Detailed information of the identified candidate genes associated with flowering pathways of Isatis indigotica.

\begin{tabular}{|c|c|}
\hline Gene name & Unigenes \\
\hline AP1 & 3 \\
\hline AP2 & 1 \\
\hline AGL6 & 1 \\
\hline AGL8 & 1 \\
\hline AGL14 & 1 \\
\hline AGL15 & 1 \\
\hline AGL16 & 1 \\
\hline AGL17 & 1 \\
\hline AGL18 & 1 \\
\hline AGL19 & 1 \\
\hline CAL & 2 \\
\hline CCA1 & 1 \\
\hline $\mathrm{CO}$ & 2 \\
\hline COL9 & 1 \\
\hline CHS & 2 \\
\hline CRY1 & 3 \\
\hline CRY2 & 1 \\
\hline EBS & 3 \\
\hline ELF3 & 1 \\
\hline ELF6 & 4 \\
\hline ELF8 & 1 \\
\hline ELF9 & 2 \\
\hline EMF1 & 1 \\
\hline EMF2 & 1 \\
\hline ESD4 & 1 \\
\hline FD & 1 \\
\hline FKF1 & 1 \\
\hline FLC & 1 \\
\hline FLD & 1 \\
\hline FLK & 3 \\
\hline
\end{tabular}

$\begin{array}{ll}\text { FPA } & 1 \\ \text { FPF } & 2 \\ \text { FT } & 1 \\ \text { GI } & 2 \\ \text { HUA2 } & \\ \text { LD } & 1\end{array}$

$\begin{array}{ll}\text { LHY } & 1 \\ \text { LKP2 } & 1\end{array}$

TRINITY_DN44529_c0_g2 TRINITY_DN59583_c0_g1 TRINITY_DN60394_c3_g1 TRINITY_DN64890_c0_g1 TRINITY_DN62832_c0_g1 TRINITY_DN48318_c3_g1 TRINITY_DN68423_c2_g1 TRINITY_DN18713_c0_g1 TRINITY_DN69985_c0_g2 TRINITY_DN59446_c0_g1 TRINITY_DN58069_c0_g1 TRINITY_DN68423_c2_g2 TRINITY_DN46328_c0_g1 TRINITY_DN60394_c3_g2 TRINITY_DN68029_c0_g1 TRINITY_DN63019_c0_g1 TRINITY_DN63019_c0_g2 TRINITY_DN65596_c1_g1 TRINITY_DN53874_c0_g1 TRINITY_DN61788_c0_g1 TRINITY_DN29253_c0_g1 TRINITY_DN45325_c0_g1 TRINITY_DN66742_c0_g1 TRINITY_DN67410_c0_g1 TRINITY_DN69128_c1_g2 TRINITY_DN62817_c0_g1 TRINITY_DN50493_c0_g1 TRINITY_DN58990_c1_g1 TRINITY_DN69071_c0_g2 TRINITY_DN51551_c0_g1 TRINITY_DN69458_c0_g3 TRINITY_DN95956_c0_g1 TRINITY_DN69534_c0_g2 TRINITY_DN67027_c0_g1 TRINITY_DN67027_c0_g2 TRINITY_DN57304_c0_g1 TRINITY_DN66245_c2_g3 TRINITY_DN70231_c1_g3 TRINITY_DN64502_c0_g2 TRINITY_DN69998_c3_g1 TRINITY_DN69832_c1_g1 TRINITY_DN67304_c0_g1 TRINITY_DN40841_c0_g3 TRINITY_DN40841_c0_g2 TRINITY_DN40841_c0_g1 TRINITY_DN70605_c0_g2 TRINITY_DN60601_c0_g2 TRINITY_DN47820_c0_g1 TRINITY_DN67831_c2_g1 TRINITY_DN67682_c0_g1 TRINITY_DN84722_c0_g1 TRINITY_DN69595_c0_g1 TRINITY_DN69302_c0_g3 TRINITY_DN69302_c0_g1 TRINITY_DN69302_c0_g2 TRINITY_DN68323_c1_g1 TRINITY_DN63261_c1_g1

Continued next page
Supplemental Table 3. Continued.

\begin{tabular}{|c|c|c|}
\hline Gene name & Unigenes (no.) & Gene ID \\
\hline \multirow{3}{*}{$\overline{\text { PHYA }}$} & 3 & TRINITY_DN31883_c0_g1 \\
\hline & & TRINITY_DN76871_c0_g1 \\
\hline & & TRINITY_DN76871_c0_g2 \\
\hline PHYB & 1 & TRINITY_DN68291_c2_g2 \\
\hline \multirow[t]{7}{*}{ PHYD } & 7 & TRINITY_DN27276_c0_g1 \\
\hline & & TRINITY_DN27276_c0_g2 \\
\hline & & TRINITY_DN68291_c0_g1 \\
\hline & & TRINITY_DN68291_c0_g2 \\
\hline & & TRINITY_DN68291_c1_g4 \\
\hline & & TRINITY_DN68291_c2_g1 \\
\hline & & TRINITY_DN68291_c3_g1 \\
\hline PHYE & 1 & TRINITY_DN69481_c0_g1 \\
\hline PIE1 & 1 & TRINITY_DN67850_c0_g2 \\
\hline \multirow[t]{2}{*}{ PIF3 } & 2 & TRINITY_DN46533_c0_g1 \\
\hline & & TRINITY_DN46533_c0_g2 \\
\hline \multirow[t]{3}{*}{ SEP1 } & 3 & TRINITY_DN44852_c0_g2 \\
\hline & & TRINITY_DN51213_c0_g1 \\
\hline & & TRINITY_DN51213_c0_g2 \\
\hline SOC1 & 1 & TRINITY_DN38410_c0_g3 \\
\hline SPA & 1 & TRINITY_DN69209_c0_g2 \\
\hline TEM1 & 1 & TRINITY_DN52784_c0_g2 \\
\hline \multirow[t]{2}{*}{ TFL1 } & 2 & TRINITY_DN33790_c0_g1 \\
\hline & & TRINITY_DN21486_c0_g1 \\
\hline TFL2 & 1 & TRINITY_DN61367_c0_g1 \\
\hline \multirow[t]{4}{*}{ TOC1 } & 4 & TRINITY_DN70446_c2_g2 \\
\hline & & TRINITY_DN34145_c0_g1 \\
\hline & & TRINITY_DN55625_c0_g1 \\
\hline & & TRINITY_DN55625_c0_g2 \\
\hline VEL1 & 1 & TRINITY_DN64509_c0_g1 \\
\hline \multirow[t]{2}{*}{ VRN1 } & 2 & TRINITY_DN53167_c0_g1 \\
\hline & & TRINITY_DN52604_c0_g1 \\
\hline VRN2 & 1 & TRINITY_DN21929_c0_g1 \\
\hline \multirow[t]{2}{*}{ WNK1 } & 2 & TRINITY_DN33663_c0_g1 \\
\hline & & TRINITY_DN54268_c0_g1 \\
\hline
\end{tabular}


Supplemental Table 4. List of gene-specific primers used in quantitative real time polymerase chain reaction (qRT-PCR).

\begin{tabular}{llr}
\hline Gene & \multicolumn{1}{c}{ Sense primer $\left(5^{\prime}-3^{\prime}\right)$} & Anti-sense primer $\left(5^{\prime}-3^{\prime}\right)$ \\
\hline ELF6 & TTAAGGCACATGCGGCTAT & TTTGAGGTCCAGTCTACGC \\
ELF8 & TGGATCTCATAAGCGTAAGG & TTAGTGTTGGCGTCTTCGT \\
EMF2 & CGGCGTGCTATTAGGAATC & AGTCTTGCCAACAGGATAT \\
PIE1 & AAAGCCGTCTGACAGTATGG & TCCTGAGGCAACCAAGAAT \\
AGL15 & CCTTGCGTAGACAGGTTCA & ACGAGTGGGTTCTTGGGAT \\
AGL17 & CCAATACCGACAAGCTCTA & ATAGTTGTGCTGCTCCAT \\
TFL1 & GGGAAGAGTGGTCGGAGAT & GAGGAGACAGACGAAGGGA \\
FLC & CTTGGATCGTCAGTCGAAATG & GGGCGTTCTCAAGGTGGTC \\
\hline
\end{tabular}

